\title{
Entanglement transfer from quantum matter to classical geometry in an emergent holographic dual description of a scalar field theory
}

\author{
Ki-Seok Kim ${ }^{a, b}$ and Shinsei Ryu ${ }^{c}$ \\ ${ }^{a}$ Department of Physics, POSTECH, Pohang, Gyeongbuk 37673, South Korea \\ ${ }^{b}$ Asia Pacific Center for Theoretical Physics (APCTP), Pohang, Gyeongbuk 37673, South Korea \\ ${ }^{c}$ Kadanoff Center for Theoretical Physics, University of Chicago, IL 60637, U.S.A. \\ E-mail: tkfkd@postech.ac.kr, shinseir@princeton.edu
}

ABSTRACT: Applying recursive renormalization group transformations to a scalar field theory, we obtain an effective quantum gravity theory with an emergent extra dimension, described by a dual holographic Einstein-Klein-Gordon type action. Here, the dynamics of both the dual order-parameter field and the metric tensor field originate from densitydensity and energy-momentum tensor-tensor effective interactions, respectively, in the recursive renormalization group transformation, performed approximately in the Gaussian level. This linear approximation in the recursive renormalization group transformation for the gravity sector gives rise to a linearized quantum Einstein-scalar theory along the $z$-directional emergent space. In the large $N$ limit, where $N$ is the flavor number of the original scalar fields, quantum fluctuations of both dynamical metric and dual scalar fields are suppressed, leading to a classical field theory of the Einstein-scalar type in $(D+1)$-spacetime dimensions. We show that this emergent background gravity describes the renormalization group flows of coupling functions in the UV quantum field theory through the extra dimension. More precisely, the IR boundary conditions of the gravity equations correspond to the renormalization group $\beta$-functions of the quantum field theory, where the infinitesimal distance in the extra-dimensional space is identified with an energy scale for the renormalization group transformation. Finally, we also show that this dual holographic formulation describes quantum entanglement in a geometrical way, encoding the transfer of quantum entanglement from quantum matter to classical gravity in the large $N$ limit. We claim that this entanglement transfer serves as a microscopic foundation for the emergent holographic duality description.

Keywords: AdS-CFT Correspondence, Renormalization Group, Holography and condensed matter physics (AdS/CMT), Resummation

ArXiv EPrint: 2003.00165 


\section{Contents}

1 Introduction $\quad 2$

2 Overview 3

2.1 Emergent gravity description for a scalar field theory 3

2.2 Transferring quantum entanglement from matter to geometry 6

3 Derivation of the holographic Landau-Ginzburg effective field theory $\quad 7$

3.1 The first renormalization group transformation in real space a la Polchinski 7

3.2 Recursive renormalization group transformations a la Sung-Sik Lee 12

3.3 Remarks on the renormalization group transformation for the metric tensor 16

4 Physical interpretation of the emergent dynamical metric tensor: from renormalization group equations of coupling parameters to the evolution equation of the metric tensor

5 Evaluation of entanglement entropy based on the heat kernel method 23

5.1 Entanglement entropy 23

5.2 Heat kernel method for entanglement entropy 26

5.3 Invariance of entanglement entropy with respect to renormalization group transformations

6 Discussion $\quad 29$

6.1 Remarks on the appearance of higher-spin fields 29

6.2 Remarks on the linear approximation for the dynamics of the metric tensor in the recursive renormalization group transformation 32

$\begin{array}{lll}7 & \text { Conclusion } & 36\end{array}$

A Emergent geometric description for a scalar lattice field theory in one dimension

B Further developments for the invariance of entanglement entropy with respect to renormalization group transformations

B.1 An identity for entanglement entropy at $z=z_{f} \quad 43$

B.2 Entanglement entropy after the first iteration 


\section{Introduction}

Renormalization group transformations lead us to construct an effective field theory of renormalized low-energy fluctuations in terms of renormalized interaction vertices, which result from quantum fluctuations of high-energy modes [1]. This renormalization group analysis requires that the resulting effective field theory remains invariant in its mathematical form after the renormalization group transformation. As a result, we can extract out how interaction vertices evolve as a function of the renormalization group energy scale, referred to as renormalization group $\beta$-functions. In addition, we obtain the so-called Callan-Symanzik equation that a correlation function has to satisfy in the renormalization group transformation [1]. Solving this renormalization group equation for the correlation function, we find a renormalized correlation function in terms of transfer momentum and energy, which encodes anomalous scaling dimensions of renormalized low-energy fluctuations and renormalization group $\beta$-functions of interaction vertices.

One may suggest that the renormalization group energy scale is introduced into an effective field theory as an extra dimension, manifesting a renormalization group flow of the effective field theory through the extra dimensional space. Renormalization group flows of interaction vertices are realized along the extra dimension, serving as an effective curved spacetime for renormalized low-energy fluctuations in this effective field theory. Renormalization group flows of correlation functions can be described by introducing external source fields, coupled to conserved currents minimally, into the effective field theory, regarded to be a dual description in this emergent spacetime with an extra dimension. This novel construction for renormalization group transformations reminds us of the holographic duality conjecture [2-8].

In this study, we follow this line of thoughts and suggest a microscopic foundation for the holographic duality conjecture [9-29]. The key idea is the recursive applications of the renormalization group transformations. We follow the real space renormalization group scheme developed by Polchinski (although whether one works in real or momentum space should not matter ultimately). This renormalization group technique was further developed by Sung-Sik Lee, by applying the renormalization group transformations in a recursive way [31-33]. Resorting to this renormalization group analysis, we show that renormalization group flows of interaction vertices appear to form an emergent curved spacetime with an extra dimension. Here, the infinitesimal distance in this extra-dimensional space is identified with the renormalization group energy scale. To be concrete, we apply the Polchinski renormalization group technique a la Sung-Sik Lee to a quantum field theory of bosons with self-interactions in $D$-spacetime dimensions. We derive an effective dual holographic description of the Einstein-Klein-Gordon type theory in $(D+1)$-spacetime dimensions, given by a quantum gravity theory of a modified Einstein-Hilbert action coupled to fluctuating scalar fields dual to the density of the original bosons. It turns out that quantum fluctuations of both dynamical metric and dual scalar fields are suppressed in the large $N$ limit, where $N$ is the number of boson flavors. As a result, we obtain a classical field theory of the Einstein-scalar type in $(D+1)$-spacetime dimensions. 
Since the quantum field theory is geometrized and described by a classical field theory, it is natural to ask how the quantum mechanical nature of the original theory can be encoded into the classical and geometric description appropriately. We thus study the entanglement entropy [34-39], which measures the quantum correlation between a given spatial region and its complement, by utilizing the classical holographic description. As we will show, it turns out that entanglement is transferred from quantum matter to classical gravity through recursive renormalization group transformations. We claim that the entanglement transfer serves as a microscopic foundation for the holographic duality conjecture.

\section{Overview}

\subsection{Emergent gravity description for a scalar field theory}

Since the present study involves a lot of formulae, discussing our main results briefly with the introduction of the structure of this paper would be helpful for readers to figure out the physics. We start from an effective field theory of self-interacting bosons, where the partition function is

$$
Z=\int D \phi_{\alpha}(x) \exp \left\{-S_{\mathrm{UV}}\left[\phi_{\alpha}(x) ; g_{\mu \nu}^{B}(x)\right]\right\}
$$

and the effective action is

$$
\begin{aligned}
& S_{\mathrm{UV}}\left[\phi_{\alpha}(x) ; g_{\mu \nu}^{B}(x)\right] \\
& \quad=\int d^{D} x \sqrt{g_{B}}\left\{g_{B}^{\mu \nu}\left(\partial_{\mu} \phi_{\alpha}\right)\left(\partial_{\nu} \phi_{\alpha}\right)+m^{2} \phi_{\alpha}^{2}+\xi R_{B} \phi_{\alpha}^{2}+\frac{u}{2 N} \phi_{\alpha}^{2} \phi_{\beta}^{2}+\frac{\lambda}{2 N} T_{\mu \nu} T^{\mu \nu}\right\} .
\end{aligned}
$$

Here, $\phi_{\alpha}(x)$ is a real scalar field with a flavor index $\alpha=1, \ldots, N$ at a $D$-dimensional spacetime coordinate $x$. We point out that the two flavor indices $\alpha$ and $\beta$ are summed independently. $g_{B}^{\mu \nu}$ with $\mu \& \nu=0, \ldots, D-1$ is a background metric tensor to describe a $D$ dimensional curved spacetime manifold, where the bosonic field lives. $g_{B}$ is the determinant of the metric tensor, where $d^{D} x \sqrt{g_{B}}$ gives an invariant volume factor. For example, we may consider the background metric as follows

$$
d s^{2}=d r^{2}+r^{2} d \theta^{2}+\delta_{i j} d x_{\perp}^{i} d x_{\perp}^{j}, \quad g_{r r}^{B}=1, \quad g_{\theta \theta}^{B}=r^{2}, \quad g_{i j}^{B \perp}=\delta_{i j}, \quad \sqrt{g_{B}}=r,
$$

which will be useful for evaluation of the entanglement entropy using the replica technique. Here, $i$ and $j$ run from 2 to $D-1$. $m$ denotes the mass of these scalar bosons and $u$ represents their self-interactions. $R_{B}$ is the Ricci scalar and $\xi$ is a coupling constant between scalar fields and the background curvature [40]. Finally, the last term describes effective interactions between energy-momentum tensor currents $T^{\mu \nu}$, where $\lambda$ is the corresponding coupling constant. Although these effective interactions are irrelevant at both Gaussian and Wilson-Fisher fixed points in the perturbative regime [1], we introduce this term explicitly, which plays an important role in uplifting the background geometry into full dynamical degrees of freedom for the emergent bulk geometry [41].

In section 3, by implementing the Polchinski real-space renormalization group technique [30] to this effective field theory in a recursive way a la Sung-Sik Lee [31-33], we 
obtain a quantum gravity theory coupled to a dual scalar field in one-dimensional higher spacetime. It is given by the following partition function

$$
\begin{aligned}
Z= & Z_{\Lambda} \int D \varphi(x, z) D g_{\mu \nu}(x, z) \exp \left\{-S_{\mathrm{UV}}\left[g_{\mu \nu}(x, 0), \varphi(x, 0)\right]-S_{\mathrm{IR}}\left[g_{\mu \nu}\left(x, z_{f}\right), \varphi\left(x, z_{f}\right)\right]\right. \\
& \left.-S_{\mathrm{Bulk}}\left[g_{\mu \nu}(x, z), \varphi(x, z)\right]\right\} .
\end{aligned}
$$

Here, $z$ is the coordinate of an emergent extra dimension, identified with a renormalization group scale as discussed in the introduction. $g_{\mu \nu}(x, z)$ with $\mu \& \nu=0, \ldots, D-1$ is an emergent dynamical metric field, where metric tensors involved with this extra dimension are gauge-fixed as $g_{D D}(x, z)=1$ and $g_{\mu D}(x, z)=0 . \varphi(x, z)$ is a dual scalar field, conventionally taken to be $\varphi(x)=\frac{1}{N}\left\langle\sum_{\alpha=1}^{N}\left[\phi_{\alpha}(x)\right]^{2}\right\rangle$ in the large $N$ analysis of the quantum field theory [42], where $\langle\hat{O}\rangle$ is the expectation value (ensemble average) of the operator $\hat{O}$ at the Gaussian fixed point.

The dynamics of both emergent metric and dual scalar fields are governed by the following bulk effective action

$$
\begin{aligned}
& S_{\mathrm{Bulk}}\left[g_{\mu \nu}(x, z), \varphi(x, z)\right]=N \int_{0}^{z_{f}} d z \int d^{D} x \sqrt{g(x, z)}\{ \\
& +\frac{1}{2 u}\left[\partial_{z} \varphi(x, z)\right]^{2}+\frac{\mathcal{C}_{\varphi}}{2} g^{\mu \nu}(x, z)\left[\partial_{\mu} \varphi(x, z)\right]\left[\partial_{\nu} \varphi(x, z)\right]+\mathcal{C}_{\xi} R(x, z)[\varphi(x, z)]^{2} \\
& \quad-\frac{1}{2 \lambda}\left(\partial_{z} g^{\mu \nu}(x, z)-g^{\mu \nu^{\prime}}(x, z)\left(\partial_{\nu^{\prime}} \partial_{\mu^{\prime}} G_{x x^{\prime}}\left[g_{\mu \nu}(x, z), \varphi(x, z)\right]\right)_{x^{\prime} \rightarrow x} g^{\mu^{\prime} \nu}(x, z)\right)^{2} \\
& \left.+\frac{1}{2 \kappa}(R(x, z)-2 \Lambda)\right\} .
\end{aligned}
$$

Here, the dynamics of $\varphi(x, z)$ is given by rather a conventional form of the bosonic effective action on a curved spacetime manifold [40]. $\mathcal{C}_{\varphi}$ and $\mathcal{C}_{\xi}$ are positive constants, which decrease as the mass of the original scalar fields increases. The metric-tensor field is described essentially by the Einstein-Hilbert type action in a gauge-fixed version, which originates from quantum fluctuations of matter fields. This emergent Einstein-Hilbert action is analogous to the notion of induced gravity [43,44]. We also point out that the dynamics of the metric along the extra dimensional space is modified through $G_{x x^{\prime}}\left[g_{\mu \nu}(x, z), \varphi(x, z)\right]$, which is the Green's function given by quantum fluctuations of heavy-mass scalar fields in the renormalization group transformation,

$$
\begin{aligned}
& \left\{-\frac{1}{\sqrt{g(x, z)}} \partial_{\mu}\left(\sqrt{g(x, z)} g^{\mu \nu}(x, z) \partial_{\nu}\right)+\frac{1}{2 d z}\left[m^{2}-i \varphi(x, z)\right]\right\} G_{x x^{\prime}}\left[g_{\mu \nu}(x, z), \varphi(x, z)\right] \\
& =\frac{1}{\sqrt{g(x, z)}} \delta^{(D)}\left(x-x^{\prime}\right)
\end{aligned}
$$

where $d z$ is an infinitesimal parameter of the renormalization group transformation. The evolution dynamics of both metric and dual scalar fields are purely determined by this Green's function in addition to the emergent local symmetry in the dynamical bulk geometry, i.e., $(D+1)$-dimensional diffeomorphism invariance [31-33], although it is explicitly broken by the gauge fixing. In section 6 , we argue that the dynamics of the metric tensor 
in the renormalization group flow along the extra-dimensional space may be interpreted as introduction of a higher curvature term into the Einstein-Hilbert action, combined with the Ricci flow and the holographic renormalization group. As clearly seen in this expression, quantum fluctuations of both metric and dual scalar fields are suppressed in the large $N$ limit. As a result, both dynamics are described by coupled classical equations of motion in the presence of the emergent extra dimensional space.

Since these coupled equations of motion are second order in derivatives with respect to the extra dimensional space, we need two boundary conditions. The first boundary condition is described by an effective UV action in eq. (2.4), given by,

$$
\begin{aligned}
S_{\mathrm{UV}}\left[g_{\mu \nu}(x, 0), \varphi(x, 0)\right]= & N \int d^{D} x \sqrt{g(x, 0)} \\
& \times\left\{\frac{1}{2 u}(\varphi(x, 0)-i \xi R(x, 0))^{2}-\frac{1}{2 \lambda}\left(g^{\mu \nu}(x, 0)-g_{B}^{\mu \nu}(x)\right)^{2}\right\} .
\end{aligned}
$$

Of course, an actual equation corresponding to this UV boundary condition has to take into account a linear $z$ derivative term at $z=0$, which originates from the above bulk effective action by the technique of integration-by-parts $[45,46]$. The other boundary condition is provided by an effective IR action

$$
\begin{aligned}
S_{\mathrm{IR}} & {\left[g_{\mu \nu}\left(x, z_{f}\right), \varphi\left(x, z_{f}\right)\right] } \\
= & N \int d^{D} x \sqrt{g\left(x, z_{f}\right)}\left\{\frac{\mathcal{C}_{\varphi}^{f}}{2} g^{\mu \nu}\left(x, z_{f}\right)\left[\partial_{\mu} \varphi\left(x, z_{f}\right)\right]\left[\partial_{\nu} \varphi\left(x, z_{f}\right)\right]+\mathcal{C}_{\xi}^{f} R\left(x, z_{f}\right)\left[\varphi\left(x, z_{f}\right)\right]^{2}\right. \\
& \left.+\frac{1}{2 \kappa_{f}}\left(R\left(x, z_{f}\right)-2 \Lambda_{f}\right)\right\},
\end{aligned}
$$

where the original scalar fields have been integrated out, using the gradient expansion, to give this effective action for the dual scalar fields and metric tensors [40, 43, 44] at $z=z_{f}$. Here, $\mathcal{C}_{\varphi}^{f}$ and $\mathcal{C}_{\xi}^{f}$ are positive coefficients, which also decrease as the mass $m$ increases. The effective gravitational constant $\kappa_{f}$ and the cosmological parameter $\Lambda_{f}$ are similarly determined from the gradient expansion. Also, actual equations to describe the IR boundary condition have to be supported by the linear $z$-derivative term at $z=z_{f}$. All these boundary conditions including the Gibbons-Hawking-York term are self-consistently determined by these effective actions. We show all derivations for this emergent holographic construction in section 3 .

$z_{f}$ in eqs. (2.5) and (2.8) is an IR cutoff, which may be regarded to be inversely proportional to temperature in the renormalization group transformation. This implies that the IR boundary conditions correspond to renormalization group equations. More precisely, as we discuss in section 4 and appendix A, the IR boundary equations for metric tensors correspond to renormalization group $\beta$-functions of interaction vertices. On the other hand, the IR boundary conditions of dual scalar fields does to a dual expression of the Callan-Symanzik equation.

Finally, we point out that the limit $z_{f} \rightarrow 0$ leads to the conventional large $N$ analysis as follows

$$
S_{\mathrm{UV}}\left[g_{\mu \nu}(x, 0), \varphi(x, 0)\right]+\lim _{z_{f} \rightarrow 0} S_{\mathrm{IR}}\left[g_{\mu \nu}\left(x, z_{f}\right), \varphi\left(x, z_{f}\right)\right]=S_{\mathrm{UV}}\left[g_{\mu \nu}^{B}(x), \varphi(x)\right],
$$


where the bulk effective action vanishes in the left-hand-side of the equality and the original scalar fields are integrated out to give an effective action in the large $N$ limit for the righthand-side of the equality.

\subsection{Transferring quantum entanglement from matter to geometry}

To figure out how the quantum-entanglement structure can be translated into the emergent classical geometry, we calculate in section 5, the entanglement entropy [34-39]. Entanglement entropy may be regarded as an entropy of a subsystem, given by the von-Neumann entropy of the reduced density matrix for the subsystem. Here, we perform a field-theory calculation of the entanglement entropy, based on the replica technique in a Riemann surface with a conical singularity. It turns out that the entanglement entropy at the IR cutoff $z=z_{f}$ can be decomposed into $\mathcal{S}_{E E}^{\phi_{\alpha}}\left(z_{f}\right)$ of the matter and $\mathcal{S}_{E E}^{G R}\left(z_{f}\right)$ of the emergent gravity as follows

$$
\mathcal{S}_{E E}\left(z_{f}\right)=\mathcal{S}_{E E}^{\phi_{\alpha}}\left(z_{f}\right)+\mathcal{S}_{E E}^{G R}\left(z_{f}\right)
$$

Resorting to the heat-kernel method, one finds that the entanglement entropy of free bosons with the flavor degeneracy $N$ follows an area law, given by [47]

$$
\mathcal{S}_{E E}^{\phi_{\alpha}}\left(z_{f}\right)=\frac{N}{6(D-2)(4 \pi)^{\frac{D}{2}-1}} \frac{\mathcal{A}\left[\Sigma\left(z_{f}\right)\right]}{\epsilon^{D-2}},
$$

where

$$
\mathcal{A}\left[\Sigma\left(z_{f}\right)\right]=\int_{\Sigma\left(z_{f}\right)} d^{D-2} x_{\perp} \sqrt{\gamma\left(x, z_{f}\right)}
$$

is an area of the subsystem at $z=z_{f}$. $\epsilon$ is a UV cutoff, here corresponding to a microscopic scale for mapping from a discrete version of the holographic Einstein-Klein-Gordon type theory to the above continuum field theory eq. (2.5), as we will discuss in detail in section 3 . On the other hand, the gravity contribution to the entanglement entropy is given by

$$
\mathcal{S}_{E E}^{G R}\left(z_{f}\right)=\left.N \int d^{D-2} x_{\perp} \int_{0}^{2 \pi} d \theta \int_{0}^{\infty} d r \int_{0}^{z_{f}} d z \sqrt{g_{n}\left(r, x_{\perp}, z\right)} T_{\mu \nu, n}^{\mathrm{Bulk}}\left(r, x_{\perp}, z\right) \frac{\partial g_{n}^{\mu \nu}\left(r, x_{\perp}, z\right)}{\partial n}\right|_{n=1} .
$$

Here,

$$
T_{\mu \nu, n}^{\mathrm{Bulk}}\left(r, x_{\perp}, z\right)=\frac{1}{\sqrt{g_{n}\left(r, x_{\perp}, z\right)}} \frac{\partial \sqrt{g_{n}\left(r, x_{\perp}, z\right)} \mathcal{L}_{\mathrm{Bulk}}\left[g_{\mu \nu, n}\left(r, x_{\perp}, z\right), \varphi_{n}\left(r, x_{\perp}, z\right)\right]}{\partial g_{n}^{\mu \nu}\left(r, x_{\perp}, z\right)}
$$

is an energy-momentum tensor of a $(D+1)$-dimensional modified Einstein gravity in eq. (2.5), given by $S_{\mathrm{Bulk}}\left[g_{\mu \nu, n}(x, z), \varphi_{n}(x, z)\right]=\int_{0}^{z_{f}} d z \int d^{D} x \sqrt{g_{n}(x, z)} \mathcal{L}_{\mathrm{Bulk}}\left[g_{\mu \nu, n}(x, z)\right.$, $\left.\varphi_{n}(x, z)\right]$. The quantum average of $\left\langle T_{\mu \nu, n}^{\mathrm{Bulk}}\left(r, x_{\perp}, z\right)\right\rangle$ with respect to the quantum gravity action is reduced into its classical value of $T_{\mu \nu, n}^{g}\left(r, x_{\perp}, z\right)$ in the large $N$ limit. $n$ is the replica index to describe the conical singularity of the geometry [47]. The detailed discussion is given in section 5 . 
An important observation is that the entanglement entropy does not depend on the IR cutoff $z_{f}$ at all. In other words, it is a renormalization-group invariant, regardless of $z_{f}$. This originates from that fact that the partition function is a renormalization-group invariant. As a result, we find the renormalization-group flow equation for the entanglement entropy, given by

$$
\partial_{z_{f}} \mathcal{S}_{E E}\left(z_{f}\right)=\partial_{z_{f}} \mathcal{S}_{E E}^{\phi_{\alpha}}\left(z_{f}\right)+\partial_{z_{f}} \mathcal{S}_{E E}^{G R}\left(z_{f}\right)=0
$$

This observation leads us to conclude that the entanglement entropy of the matter sector at UV is transferred into that of the classical bulk gravity part at IR. (While we could not verify this relation based on explicit calculations for the general formula, we could check it after the first renormalization group transformation, as discussed in detail in appendix B.) Recalling that an emergent geometry of gapped quantum fields is essentially a cap geometry, where the spacetime does not exist beyond some scale in the extra dimension [33, 48, 49], the entanglement entropy of the matter sector vanishes after the cap geometry in the extra dimensional space. Our conclusion on the entanglement transfer is consistent with a recent study [50].

\section{Derivation of the holographic Landau-Ginzburg effective field theory}

\subsection{The first renormalization group transformation in real space a la Polchin- ski}

We start from an effective scalar field theory on a $D$-dimensional curved spacetime described by a background metric tensor $g_{B}^{\mu \nu}$. The partition function is given by [40]

$$
\begin{aligned}
Z= & \int D \phi_{\alpha} D g_{\mu \nu}^{(0)} D T_{\mu \nu}^{(0)} \exp \left[-\int d^{D} x \sqrt{g^{(0)}}\{\right. \\
& \left.\left.g^{\mu \nu(0)}\left(\partial_{\mu} \phi_{\alpha}\right)\left(\partial_{\nu} \phi_{\alpha}\right)+m^{2} \phi_{\alpha}^{2}+\xi R^{(0)} \phi_{\alpha}^{2}+\frac{u}{2 N} \phi_{\alpha}^{2} \phi_{\beta}^{2}-N T_{\mu \nu}^{(0)}\left(g^{\mu \nu(0)}-g_{B}^{\mu \nu}\right)\right\}\right] .
\end{aligned}
$$

Here, $T_{\mu \nu}^{(0)}$ is a Lagrange multiplier field to impose the constraint $g^{\mu \nu(0)}=g_{B}^{\mu \nu}$. For the time being, we do not consider the effective interaction between energy-momentum tensor currents, for simplicity in the presentation of derivations. To deal with self-interactions between bosons, we introduce a dual scalar field $\varphi^{(0)}$ as

$$
\begin{aligned}
Z= & \int D \phi_{\alpha} D \varphi^{(0)} D g_{\mu \nu}^{(0)} D T_{\mu \nu}^{(0)} \exp \left[-\int d^{D} x \sqrt{g^{(0)}}\{\right. \\
& \left.\left.g^{\mu \nu(0)}\left(\partial_{\mu} \phi_{\alpha}\right)\left(\partial_{\nu} \phi_{\alpha}\right)+m^{2} \phi_{\alpha}^{2}+\xi R^{(0)} \phi_{\alpha}^{2}-i \varphi^{(0)} \phi_{\alpha}^{2}+\frac{N}{2 u} \varphi^{(0) 2}-N T_{\mu \nu}^{(0)}\left(g^{\mu \nu(0)}-g_{B}^{\mu \nu}\right)\right\}\right]
\end{aligned}
$$

by the Hubbard-Stratonovich transformation. 
To implement the renormalization group transformation for the dynamics of dual scalar fields, we introduce an auxiliary field $\eta^{(0)}$ in the following way:

$$
\begin{aligned}
Z= & \int D \phi_{\alpha} D \varphi^{(0)} D \eta^{(0)} D g_{\mu \nu}^{(0)} D T_{\mu \nu}^{(0)} \exp \left[-\int d^{D} x \sqrt{g^{(0)}}\{\right. \\
& g^{\mu \nu(0)}\left(\partial_{\mu} \phi_{\alpha}\right)\left(\partial_{\nu} \phi_{\alpha}\right)+m^{2} \phi_{\alpha}^{2}+\xi R^{(0)} \phi_{\alpha}^{2}-i \varphi^{(0)} \phi_{\alpha}^{2} \\
& \left.\left.+\frac{N}{2 u} \varphi^{(0) 2}+\frac{N}{2 u_{\eta}} \eta^{(0) 2}-N T_{\mu \nu}^{(0)}\left(g^{\mu \nu(0)}-g_{B}^{\mu \nu}\right)\right\}\right] .
\end{aligned}
$$

We emphasize that the introduction of this auxiliary field does not change any physics except for the normalization constant of the partition function, omitted here for notational simplicity.

Now, we separate low-energy and high-energy dual scalar fields, $\varphi^{(0)}$ and $\chi^{(0)}$, respectively, as follows

$$
\varphi^{(0)} \Longrightarrow \varphi^{(0)}+\chi^{(0)}, \quad \eta^{(0)} \Longrightarrow c_{\varphi}^{(0)} \varphi^{(0)}+c_{\chi}^{(0)} \chi^{(0)}
$$

Here, we determine two coefficients of $c_{\varphi}^{(0)}$ and $c_{\chi}^{(0)}$ as

$$
c_{\varphi}^{(0)}=\frac{u^{-1}}{\mu^{(0)} u_{\eta}^{-1 / 2}}, \quad c_{\chi}^{(0)}=-\frac{\mu^{(0)}}{u_{\eta}^{-1 / 2}}, \quad \mu^{(0)}=\frac{u^{-1 / 2}}{\sqrt{e^{2 \beta^{(0)}} d z-1}},
$$

such that we do not generate mixing terms between such light and heavy dual scalar fields in the mass sector. $\mu^{(0)}$ is an effective mass for the heavy dual scalar field, clarified soon. $\beta^{(0)}$ represents a local speed of coarse graining for the dual scalar field, and $d z$ is an infinitesimal parameter for the renormalization group transformation, also to be clarified below. As a result, we rewrite the mass sector in terms of these light and heavy dual boson fields as follows

$$
\frac{N}{2 u} \varphi^{(0) 2}+\frac{N}{2 u_{\eta}} \eta^{(0) 2} \Longrightarrow \frac{N}{2 u} e^{2 \beta^{(0)} d z} \varphi^{(0) 2}+\frac{N}{2 u} \frac{e^{2 \beta^{(0)} d z}}{e^{2 \beta^{(0)} d z}-1} \chi^{(0) 2}
$$

Now, it is clear why we call $\chi^{(0)}$ a heavy dual scalar field, whose mass is given by $\mu^{(0)}$. Rescaling both dual scalar fields in the following way to return the mass sector to its original form,

$$
\varphi^{(0)} \Longrightarrow e^{-\beta^{(0)} d z} \varphi^{(0)}, \quad \chi^{(0)} \Longrightarrow e^{-\beta^{(0)} d z} \chi^{(0)}
$$

we rewrite the partition function in terms of these light and heavy dual scalar bosons as

$$
\begin{aligned}
Z= & \int D \phi_{\alpha} D \varphi^{(0)} D \chi^{(0)} D g_{\mu \nu}^{(0)} D T_{\mu \nu}^{(0)} \exp \left[-\int d^{D} x \sqrt{g^{(0)}}\{\right. \\
& g^{\mu \nu(0)}\left(\partial_{\mu} \phi_{\alpha}\right)\left(\partial_{\nu} \phi_{\alpha}\right)+m^{2} \phi_{\alpha}^{2}+\xi R^{(0)} \phi_{\alpha}^{2} \\
& \left.\left.-i e^{-\beta^{(0)} d z}\left(\varphi^{(0)}+\chi^{(0)}\right) \phi_{\alpha}^{2}+\frac{N}{2 u} \varphi^{(0) 2}+\frac{N}{2 u} \frac{1}{e^{2 \beta^{(0)}} d z-1} \chi^{(0) 2}-N T_{\mu \nu}^{(0)}\left(g^{\mu \nu(0)}-g_{B}^{\mu \nu}\right)\right\}\right] .
\end{aligned}
$$


It is straightforward to perform the Gaussian integration for the heavy dual scalar field, resulting in the partition function

$$
\begin{aligned}
Z= & \int D \phi_{\alpha} D \varphi^{(0)} D g_{\mu \nu}^{(0)} D T_{\mu \nu}^{(0)} \exp \left[-\frac{1}{2} \operatorname{tr}_{x x^{\prime}} \ln \frac{N}{2 u} \frac{1}{e^{2 \beta^{(0)}} d z-1}\right. \\
& -\int d^{D} x \sqrt{g^{(0)}}\left\{g^{\mu \nu(0)}\left(\partial_{\mu} \phi_{\alpha}\right)\left(\partial_{\nu} \phi_{\alpha}\right)+m^{2} \phi_{\alpha}^{2}+\xi R^{(0)} \phi_{\alpha}^{2}-i e^{-\beta^{(0)}} d z \varphi^{(0)} \phi_{\alpha}^{2}+\frac{N}{2 u} \varphi^{(0) 2}\right. \\
& \left.\left.+\frac{u}{2 N}\left(1-e^{-2 \beta^{(0)}} d z\right) \phi_{\alpha}^{2} \phi_{\beta}^{2}-N T_{\mu \nu}^{(0)}\left(g^{\mu \nu(0)}-g_{B}^{\mu \nu}\right)\right\}\right]
\end{aligned}
$$

where self-interactions between the original scalar bosons are generated, as expected. To prepare for the second renormalization group transformation, we perform the HubbardStratonovich transformation once again for this newly generated self-interaction term,

$$
\begin{aligned}
Z= & \int D \phi_{\alpha} D \varphi^{(0)} D \varphi^{(1)} D g_{\mu \nu}^{(0)} D T_{\mu \nu}^{(0)} \exp \left[-\frac{1}{2} \operatorname{tr}_{x x^{\prime}} \ln \frac{N}{2 u} \frac{1}{e^{2 \beta^{(0)} d z}-1}\right. \\
& -\int d^{D} x \sqrt{g^{(0)}}\left\{g^{\mu \nu(0)}\left(\partial_{\mu} \phi_{\alpha}\right)\left(\partial_{\nu} \phi_{\alpha}\right)+m^{2} \phi_{\alpha}^{2}+\xi R^{(0)} \phi_{\alpha}^{2}-i e^{-\beta^{(0)}} d z \varphi^{(0)} \phi_{\alpha}^{2}+\frac{N}{2 u} \varphi^{(0) 2}\right. \\
& \left.\left.+\frac{N}{2 u} \frac{e^{2 \beta^{(0)}} d z}{e^{2 \beta^{(0)}} d z-1} \varphi^{(1) 2}-i \varphi^{(1)} \phi_{\alpha}^{2}-N T_{\mu \nu}^{(0)}\left(g^{\mu \nu(0)}-g_{B}^{\mu \nu}\right)\right\}\right]
\end{aligned}
$$

Rescaling the dual scalar field as

$$
\varphi^{(1)} \Longrightarrow e^{-\beta^{(0)} d z} \varphi^{(1)}
$$

and shifting the field as

$$
\varphi^{(1)} \Longrightarrow \varphi^{(1)}-\varphi^{(0)}
$$

together with $\varphi^{(1)} \Longrightarrow \varphi^{(1)}-i e^{-\beta^{(0)} d z} \xi R^{(0)}$ and $\varphi^{(0)} \Longrightarrow \varphi^{(0)}-i e^{-\beta^{(0)} d z} \xi R^{(0)}$, we obtain the following expression of the partition function after the first renormalization group transformation for the dual scalar-field sector

$$
\begin{aligned}
Z= & \int D \phi_{\alpha} D \varphi^{(0)} D \varphi^{(1)} D g_{\mu \nu}^{(0)} D T_{\mu \nu}^{(0)} \exp \left[-\frac{1}{2} \operatorname{tr}_{x x^{\prime}} \ln \frac{N}{2 u} \frac{1}{e^{2 \beta^{(0)} d z}-1}\right. \\
& -\int d^{D} x \sqrt{g^{(0)}}\left\{g^{\mu \nu(0)}\left(\partial_{\mu} \phi_{\alpha}\right)\left(\partial_{\nu} \phi_{\alpha}\right)+m^{2} \phi_{\alpha}^{2}-i e^{-\beta^{(0)} d z} \varphi^{(1)} \phi_{\alpha}^{2}\right. \\
& \left.\left.+\frac{N}{2 u}\left(\varphi^{(0)}-i e^{-\beta^{(0)} d z} \xi R^{(0)}\right)^{2}+\frac{N}{2 u} \frac{1}{e^{2 \beta^{(0)}} d z-1}\left(\varphi^{(1)}-\varphi^{(0)}\right)^{2}-N T_{\mu \nu}^{(0)}\left(g^{\mu \nu(0)}-g_{B}^{\mu \nu}\right)\right\}\right] .
\end{aligned}
$$

Now, we perform the first renormalization group transformation for the original boson sector. As in the renormalization group transformation for the dual scalar-field sector, we 
introduce an auxiliary field $\psi_{\alpha}$ into the partition function

$$
\begin{aligned}
Z= & \int D \phi_{\alpha} D \psi_{\alpha} D \varphi^{(0)} D \varphi^{(1)} D g_{\mu \nu}^{(0)} D T_{\mu \nu}^{(0)} \exp \left[-\frac{1}{2} \operatorname{tr}_{x x^{\prime}} \ln \frac{N}{2 u} \frac{1}{e^{2 \beta^{(0)} d z}-1}\right. \\
& -\int d^{D} x \sqrt{g^{(0)}}\left\{g^{\mu \nu(0)}\left(\partial_{\mu} \phi_{\alpha}\right)\left(\partial_{\nu} \phi_{\alpha}\right)+m^{2} \phi_{\alpha}^{2}-i e^{-\beta^{(0)} d z} \varphi^{(1)} \phi_{\alpha}^{2}+M^{2} \psi_{\alpha}^{2}\right. \\
& \left.\left.+\frac{N}{2 u}\left(\varphi^{(0)}-i e^{-\beta^{(0)} d z} \xi R^{(0)}\right)^{2}+\frac{N}{2 u} \frac{1}{e^{2 \beta^{(0)}} d z-1}\left(\varphi^{(1)}-\varphi^{(0)}\right)^{2}-N T_{\mu \nu}^{(0)}\left(g^{\mu \nu(0)}-g_{B}^{\mu \nu}\right)\right\}\right] .
\end{aligned}
$$

We point out again that the introduction of the auxiliary field $\psi_{\alpha}$ does not change any physics except for the normalization constant of the partition function, omitted here for notational simplicity.

We separate low-energy and high-energy scalar fields, $\phi_{\alpha}$ and $\Phi_{\alpha}$, respectively,

$$
\phi_{\alpha} \Longrightarrow \phi_{\alpha}+\Phi_{\alpha}, \quad \psi_{\alpha} \Longrightarrow c_{\phi}^{(0)} \phi_{\alpha}+c_{\Phi}^{(0)} \Phi_{\alpha}
$$

where two coefficients of $c_{\phi}^{(0)}$ and $c_{\Phi}^{(0)}$ are given by

$$
c_{\phi}^{(0)}=\frac{\left(m^{2}-i e^{-\beta^{(0)} d z} \varphi^{(1)}\right)}{\mu^{(0)} M}, \quad c_{\Phi}^{(0)}=-\frac{\mu^{(0)}}{M}, \quad \mu^{(0)}=\frac{\left(m^{2}-i e^{-\beta^{(0)} d z} \varphi^{(1)}\right)^{1 / 2}}{\sqrt{e^{2 \alpha^{(0)}} d z-1}} .
$$

Here, as $\beta^{(0)}, \alpha^{(0)}$ represents a local speed of coarse graining for the dual scalar field. Then, the mass sector of the original scalar fields reads

$$
\begin{aligned}
& \left(m^{2}-i e^{-\beta^{(0)} d z} \varphi^{(1)}\right) \phi_{\alpha}^{2}+M^{2} \psi_{\alpha}^{2} \\
& \Longrightarrow e^{2 \alpha^{(0)} d z}\left(m^{2}-i e^{-\beta^{(0)} d z} \varphi^{(1)}\right) \phi_{\alpha}^{2}+\frac{e^{2 \alpha^{(0)}} d z}{e^{2 \alpha^{(0)}} d z-1}\left(m^{2}-i e^{-\beta^{(0)} d z} \varphi^{(1)}\right) \Phi_{\alpha}^{2} .
\end{aligned}
$$

Rescaling both scalar fields as

$$
\phi_{\alpha} \Longrightarrow e^{-\alpha^{(0)} d z} \phi_{\alpha}, \quad \Phi_{\alpha} \Longrightarrow e^{-\alpha^{(0)} d z} \Phi_{\alpha}
$$

we rewrite the partition function in terms of light and heavy scalar fields as follows

$$
\begin{aligned}
Z= & \int D \phi_{\alpha} D \Phi_{\alpha} D \varphi^{(0)} D \varphi^{(1)} D g_{\mu \nu}^{(0)} D T_{\mu \nu}^{(0)} \exp \left[-\frac{1}{2} \operatorname{tr}_{x x^{\prime}} \ln \frac{N}{2 u} \frac{1}{e^{2 \beta^{(0)}} d z-1}\right. \\
& -\int d^{D} x \sqrt{g^{(0)}}\left\{g^{\mu \nu(0)}\left[\partial_{\mu} e^{-\alpha^{(0)} d z}\left(\phi_{\alpha}+\Phi_{\alpha}\right)\right]\left[\partial_{\nu} e^{-\alpha^{(0)}} d z\left(\phi_{\alpha}+\Phi_{\alpha}\right)\right]\right. \\
& +\left(m^{2}-i e^{-\beta^{(0)}} d z \varphi^{(1)}\right) \phi_{\alpha}^{2}+\frac{1}{e^{2 \alpha^{(0)}} d z-1}\left(m^{2}-i e^{-\beta^{(0)} d z} \varphi^{(1)}\right) \Phi_{\alpha}^{2} \\
& \left.\left.+\frac{N}{2 u}\left(\varphi^{(0)}-i e^{-\beta^{(0)} d z} \xi R^{(0)}\right)^{2}+\frac{N}{2 u} \frac{1}{e^{2 \beta^{(0)}} d z-1}\left(\varphi^{(1)}-\varphi^{(0)}\right)^{2}-N T_{\mu \nu}^{(0)}\left(g^{\mu \nu(0)}-g_{B}^{\mu \nu}\right)\right\}\right] .
\end{aligned}
$$

Here, effective couplings between light and heavy scalar bosons arise in the kinetic-energy sector. 
The next step is to perform the Gaussian integral for the heavy scalar fields. For our simple derivation, we fix a gauge for the local speed of coarse graining as

$$
\partial_{\mu} \alpha^{(0)}=0
$$

The physical meaning of this gauge fixing is that the renormalization group transformation is taken into account in a uniform way, where the coefficient in front of the infinitesimal parameter for the renormalization group transformation does not depend on the spacetime coordinate $x$. Integrating over the heavy scalar fields with this gauge fixing, we obtain

$$
\begin{aligned}
Z= & \int D \phi_{\alpha x} D \varphi_{x}^{(0)} D \varphi_{x}^{(1)} D g_{\mu \nu x}^{(0)} D T_{\mu \nu x}^{(0)} \exp \left\{-S_{\phi \phi}^{(0)}-\Delta S_{\phi \Phi}^{(0)}-S_{\Phi \Phi}^{(0)}-S_{\varphi \varphi}^{(1)}\right. \\
& \left.+N \int d^{D} x \sqrt{g_{x}^{(0)}} T_{\mu \nu x}^{(0)}\left(g_{x}^{\mu \nu(0)}-g_{B x}^{\mu \nu}\right)\right\} .
\end{aligned}
$$

Here, $S_{\phi \phi}^{(0)}$ is an effective action before the first renormalization group transformation, given by

$$
S_{\phi \phi}^{(0)}=\int d^{D} x \sqrt{g_{x}^{(0)}}\left\{e^{-2 \alpha^{(0)} d z} g_{x}^{\mu \nu(0)}\left(\partial_{\mu} \phi_{\alpha x}\right)\left(\partial_{\nu} \phi_{\alpha x}\right)+\left(m^{2}-i e^{-\beta^{(0)}} d z \varphi_{x}^{(1)}\right) \phi_{\alpha x}^{2}\right\} .
$$

$\Delta S_{\phi \Phi}^{(0)}$ is an effective action to result from the mixing terms between light and heavy scalar fields in the kinetic-energy part, given by

$$
\Delta S_{\phi \Phi}^{(0)}=-\int d^{D} x \sqrt{g_{x}^{(0)}} \int d^{D} x^{\prime} \sqrt{g_{x^{\prime}}^{(0)}} e^{-2 \alpha^{(0)} d z} g_{x}^{\mu \nu(0)} g_{x^{\prime}}^{\mu^{\prime} \nu^{\prime}(0)}\left(\partial_{\mu} \phi_{\alpha x}\right)\left(\partial_{\nu} \partial_{\mu^{\prime}} G_{x x^{\prime}}^{(0)}\right)\left(\partial_{\nu^{\prime}} \phi_{\alpha x^{\prime}}\right)
$$

where $G_{x x^{\prime}}^{(0)}$ is the Green's function of the heavy scalar bosons, and obeys

$$
\begin{aligned}
& \left\{-\frac{1}{\sqrt{g_{x}^{(0)}}} \partial_{\mu}\left(\sqrt{g_{x}^{(0)}} g_{x}^{\mu \nu(0)} \partial_{\nu}\right)+\frac{e^{\left(2 \alpha^{(0)}-\beta^{(0)}\right) d z}}{e^{2 \alpha^{(0)} d z}-1}\left(e^{-2 \alpha^{(0)} d z} m^{2}-i \varphi_{x}^{(1)}\right)\right\} G_{x x^{\prime}}^{(0)} \\
& =\frac{1}{\sqrt{g_{x}^{(0)}}} \delta^{(D)}\left(x-x^{\prime}\right) .
\end{aligned}
$$

This Green's function plays a central role in the renormalization group transformation, regarded to be the only dynamic information. $S_{\Phi \Phi}^{(0)}$ serves as an effective vacuum action to originate from quantum fluctuations of the heavy scalar fields, given by

$$
\begin{aligned}
S_{\Phi \Phi}^{(0)}= & \frac{N}{2} \operatorname{tr}_{x x^{\prime}} \ln e^{-2 \alpha^{(0)} d z} \sqrt{g_{x}^{(0)}} \\
& \times\left\{-\frac{1}{\sqrt{g_{x}^{(0)}}} \partial_{\mu}\left(\sqrt{g_{x}^{(0)}} g_{x}^{\mu \nu(0)} \partial_{\nu}\right)+\frac{e^{\left(2 \alpha^{(0)}-\beta^{(0)}\right) d z}}{e^{2 \alpha^{(0)}} d z-1}\left(e^{-2 \alpha^{(0)} d z} m^{2}-i \varphi_{x}^{(1)}\right)\right\} .
\end{aligned}
$$

Finally, $S_{\varphi \varphi}^{(1)}$ is an effective action after the first renormalization group transformation for the dual scalar bosons, given by

$$
\begin{aligned}
S_{\varphi \varphi}^{(1)}= & \frac{1}{2} \operatorname{tr}_{x x^{\prime}} \ln \frac{N}{2 u} \frac{1}{e^{2 \beta^{(0)} d z}-1} \\
& +\int d^{D} x \sqrt{g_{x}^{(0)}}\left\{\frac{N}{2 u}\left(\varphi_{x}^{(0)}-i e^{-\beta^{(0)} d z} \xi R_{x}^{(0)}\right)^{2}+\frac{N}{2 u} \frac{1}{e^{2 \beta^{(0)} d z}-1}\left(\varphi_{x}^{(1)}-\varphi_{x}^{(0)}\right)^{2}\right\} .
\end{aligned}
$$


Taking the gradient expansion in the vacuum sector of $S_{\Phi \Phi}^{(0)}$ with respect to the mass of scalar bosons and keeping all terms up to the linear order in $d z$, we obtain

$$
\begin{aligned}
Z= & \int D \phi_{\alpha x} D \varphi_{x}^{(0)} D \varphi_{x}^{(1)} D g_{\mu \nu x}^{(0)} D T_{\mu \nu x}^{(0)} \exp [ \\
& \frac{N}{2} \operatorname{tr}_{x x^{\prime}} \ln \left(2 \alpha^{(0)} d z\right)+\frac{1}{2} \operatorname{tr}_{x x^{\prime}} \ln \left(2 \beta^{(0)} d z\right)-\frac{N}{2} \operatorname{tr}_{x x^{\prime}} \ln \sqrt{g_{x}^{(0)}} m^{2}-\frac{1}{2} \operatorname{tr}_{x x^{\prime}} \ln \frac{N}{2 u} \\
& -\int d^{D} x \sqrt{g_{x}^{(0)}}\left\{\left(1-2 \alpha^{(0)} d z\right) g_{x}^{\mu \nu(0)}\left(\partial_{\mu} \phi_{\alpha x}\right)\left(\partial_{\nu} \phi_{\alpha x}\right)+\left(m^{2}-i \varphi_{x}^{(1)}\right) \phi_{\alpha x}^{2}\right. \\
& -\int d^{D} x^{\prime} \sqrt{g_{x^{\prime}}^{(0)}} g_{x}^{\mu \nu(0)} g_{x^{\prime}}^{\mu^{\prime} \nu^{\prime}(0)}\left(\partial_{\mu} \phi_{\alpha x}\right)\left(\partial_{\nu} \partial_{\mu^{\prime}} G_{x x^{\prime}}^{(0)}\right)\left(\partial_{\nu^{\prime}} \phi_{\alpha x^{\prime}}\right) \\
& +2 \alpha^{(0)} d z N\left(-\mathcal{C}_{\Lambda}+\mathcal{C}_{R} R_{x}^{(0)}+\frac{\mathcal{C}_{\varphi}}{2} g_{x}^{\mu \nu(0)}\left(\partial_{\mu} \varphi_{x}^{(1)}\right)\left(\partial_{\nu} \varphi_{x}^{(1)}\right)+\mathcal{C}_{\xi} R_{x}^{(0)} \varphi_{x}^{(1) 2}\right) \\
& \left.\left.+\frac{N}{2 u}\left(\varphi_{x}^{(0)}-i \xi R_{x}^{(0)}\right)^{2}+\beta^{(0)} d z \frac{N}{4 u}\left(\frac{\varphi_{x}^{(1)}-\varphi_{x}^{(0)}}{\beta^{(0)} d z}\right)^{2}-N T_{\mu \nu x}^{(0)}\left(g_{x}^{\mu \nu(0)}-g_{B x}^{\mu \nu}\right)\right\}\right] .
\end{aligned}
$$

We point out that the Einstein-Hilbert action appears to count vacuum fluctuations of high-energy scalar bosons in the background geometry, known to be the notion of induced gravity $[40,43,44]$. In addition, dual scalar bosons acquire their kinetic energy, where $\mathcal{C}_{\varphi}$ and $\mathcal{C}_{\xi}$ are positive constants. All the coefficients $\mathcal{C}_{\Lambda}, \mathcal{C}_{R}, \mathcal{C}_{\varphi}$, and $\mathcal{C}_{\xi}$ decrease as the mass of scalar bosons increases. The Green's function $G_{x x^{\prime}}^{(0)}$ is determined to linear order in $d z$ by the equation

$$
\left\{-\frac{1}{\sqrt{g_{x}^{(0)}}} \partial_{\mu}\left(\sqrt{g_{x}^{(0)}} g_{x}^{\mu \nu(0)} \partial_{\nu}\right)+\frac{1}{2 \alpha^{(0)} d z}\left(m^{2}-i \varphi_{x}^{(1)}\right)\right\} G_{x x^{\prime}}^{(0)}=\frac{1}{\sqrt{g_{x}^{(0)}}} \delta^{(D)}\left(x-x^{\prime}\right)
$$

\subsection{Recursive renormalization group transformations a la Sung-Sik Lee}

The idea for the second renormalization group transformation is that $S_{\phi \phi}^{(0)}+\Delta S_{\phi \Phi}^{(0)}$ is reformulated as $S_{\phi \phi}^{(1)}$, where the metric tensor is updated to be from $g^{(0) \mu \nu}$ to $g^{(1) \mu \nu}$ appropriately. However, this reformulation turns out not to be straightforward since $\Delta S_{\phi \Phi}^{(0)}$ is a nonlocal action because of $G_{x x^{\prime}}^{(0)}$, which differs from the local kinetic energy term of $S_{\phi \phi}^{(0)}$. An important observation is that this Green's function is given by an exponential form $\sim \exp \left(-\left(2 \alpha^{(0)} d z\right)^{-1 / 2} \sqrt{m^{2}-i \varphi_{x}^{(1)}}\left|x-x^{\prime}\right|\right)$, where the inverse of the decay length is $\left(2 \alpha^{(0)} d z\right)^{-1 / 2} \sqrt{m^{2}-i \varphi_{x}^{(1)}}$. As a result, we may only keep the local term in the gradient expansion as follows

$$
\left(\partial_{\nu} \partial_{\mu^{\prime}} G_{x x^{\prime}}^{(0)}\right) \approx\left(\partial_{\nu} \partial_{\mu^{\prime}} G_{x x^{\prime}}^{(0)}\right) \frac{1}{\sqrt{g_{x^{\prime}}}} \delta^{(D)}\left(x-x^{\prime}\right)+\ldots
$$

where higher gradient terms are in higher orders of $d z$. 
Taking into account this locality approximation to linear order in $d z$, we obtain

$$
\begin{aligned}
Z= & \int D \phi_{\alpha} D \varphi^{(0)} D \varphi^{(1)} D g_{\mu \nu}^{(0)} D T_{\mu \nu}^{(0)} \exp [ \\
& \frac{N}{2} \operatorname{tr}_{x x^{\prime}} \ln \left(2 \alpha^{(0)} d z\right)+\frac{1}{2} \operatorname{tr}_{x x^{\prime}} \ln \left(2 \beta^{(0)} d z\right)-\frac{N}{2} \operatorname{tr}_{x x^{\prime}} \ln \sqrt{g^{(0)}} m^{2}-\frac{1}{2} \operatorname{tr}_{x x^{\prime}} \ln \frac{N}{2 u} \\
& -\int d^{D} x \sqrt{g^{(0)}}\left\{g^{\mu \nu(0)}\left(\partial_{\mu} \phi_{\alpha}\right)\left(\partial_{\nu} \phi_{\alpha}\right)+\left(m^{2}-i \varphi^{(1)}\right) \phi_{\alpha}^{2}\right. \\
& -2 \alpha^{(0)} d z\left(g^{\mu \nu(0)}+g^{\mu \nu^{\prime}(0)}\left(\partial_{\nu^{\prime}} \partial_{\mu^{\prime}} G_{x x^{\prime}}^{(0)}\right)_{x^{\prime} \rightarrow x} g^{\mu^{\prime} \nu(0)}\right)\left(\partial_{\mu} \phi_{\alpha}\right)\left(\partial_{\nu} \phi_{\alpha}\right) \\
& +2 \alpha^{(0)} d z N\left(-\mathcal{C}_{\Lambda}+\mathcal{C}_{R} R^{(0)}+\frac{\mathcal{C}_{\varphi}}{2} g^{\mu \nu(0)}\left(\partial_{\mu} \varphi^{(1)}\right)\left(\partial_{\nu} \varphi^{(1)}\right)+\mathcal{C}_{\xi} R^{(0)} \varphi^{(1) 2}\right) \\
& \left.\left.+\frac{N}{2 u}\left(\varphi^{(0)}-i \xi R^{(0)}\right)^{2}+\beta^{(0)} d z \frac{N}{4 u}\left(\frac{\varphi^{(1)}-\varphi^{(0)}}{\beta^{(0)} d z}\right)^{2}-N T_{\mu \nu}^{(0)}\left(g^{\mu \nu(0)}-g_{B}^{\mu \nu}\right)\right\}\right]
\end{aligned}
$$

One may criticize this locality approximation for the Green's function because this approximation scheme does not take into account higher-spin fields [51-54] from the beginning. Such higher-spin fields are $O(N)$ singlets, and may arise due to the non-local reparameterization symmetry present in the quadratic action when the source for the quadratic term is promoted to a dynamical field in the bulk [55-57]. The higher-spin fields may be unavoidable because they are generated under the coarse graining even though the UV theory has only two derivatives. For example, $-\int d^{D} x \sqrt{g_{x}^{(0)}} \int d^{D} x^{\prime} \sqrt{g_{x^{\prime}}^{(0)}} g_{x}^{\mu \nu(0)} g_{x^{\prime}}^{\mu^{\prime} \nu^{\prime}(0)}\left(\partial_{\mu} \phi_{\alpha x}\right)\left(\partial_{\nu} \partial_{\mu^{\prime}} G_{x x^{\prime}}^{(0)}\right)\left(\partial_{\nu^{\prime}} \phi_{\alpha x^{\prime}}\right)$ in eq. (3.27) has a double-integration for a bi-local operator, and this can generate higher-spin operators if one reformulates this bi-local field in a local way. The derivatives in eq. (3.29) should act on the delta function, and the integration by part possibly give rise to higher-spin operators in eq. (3.30). Although this criticism does make sense in principle, we point out that our recursive renormalization group formulation keeps all the terms up to the spintwo field, neglecting the appearance of higher-spin fields. In section 6 , we argue that this dual holographic effective field theory serves as a novel mean-field theory framework with non-perturbative quantum corrections, showing that the present renormalization group formulation serves as a physically meaningful truncation scheme in the absence of higher-spin fields, where the resulting classical field theory in the large $N$ limit takes into account ! quantum corrections in the all-loop order. In particular, we show that the nonlocal diffeomorphism invariance for the origin of the appearance of higher-spin fields is explicitly broken by the existence of effective interactions.

Now, it is straightforward to update the metric tensor up to the linear order in $d z$ as follows

$$
\begin{aligned}
Z= & \int D \phi_{\alpha} D \varphi^{(0)} D \varphi^{(1)} D g_{\mu \nu}^{(0)} D T_{\mu \nu}^{(0)} D g_{\mu \nu}^{(1)} D T_{\mu \nu}^{(1)} \exp [ \\
& \frac{N}{2} \operatorname{tr}_{x x^{\prime}} \ln \left(2 \alpha^{(0)} d z\right)+\frac{1}{2} \operatorname{tr}_{x x^{\prime}} \ln \left(2 \beta^{(0)} d z\right)-\frac{N}{2} \operatorname{tr}_{x x^{\prime}} \ln \sqrt{g^{(0)}} m^{2}-\frac{1}{2} \operatorname{tr}_{x x^{\prime}} \ln \frac{N}{2 u} \\
& -\int d^{D} x \sqrt{g^{(1)}}\left\{g^{(1) \mu \nu}\left(\partial_{\mu} \phi_{\alpha}\right)\left(\partial_{\nu} \phi_{\alpha}\right)+\left(m^{2}-i \varphi^{(1)}\right) \phi_{\alpha}^{2}\right\}
\end{aligned}
$$




$$
\begin{aligned}
& -N \int d^{D} x \sqrt{g^{(0)}}\left\{\frac{1}{2 u}\left(\varphi^{(0)}-i \xi R^{(0)}\right)^{2}-T_{\mu \nu}^{(0)}\left(g^{(0) \mu \nu}-g_{B}^{\mu \nu}\right)\right\} \\
& -N \int d^{D} x \sqrt{g^{(0)}}\left\{2 \alpha^{(0)} d z\left(-\mathcal{C}_{\Lambda}+\mathcal{C}_{R} R^{(0)}+\frac{\mathcal{C}_{\varphi}}{2} g^{\mu \nu(0)}\left(\partial_{\mu} \varphi^{(1)}\right)\left(\partial_{\nu} \varphi^{(1)}\right)+\mathcal{C}_{\xi} R^{(0)} \varphi^{(1) 2}\right)\right. \\
& \left.\left.+\beta^{(0)} d z \frac{N}{4 u}\left(\frac{\varphi^{(1)}-\varphi^{(0)}}{\beta^{(0)} d z}\right)^{2}-T_{\mu \nu}^{(1)}\left(g^{(1) \mu \nu}-g^{(0) \mu \nu}-g^{(0) \mu \nu^{\prime}}\left(\partial_{\nu^{\prime}} \partial_{\mu^{\prime}} G_{x x^{\prime}}^{(0)}\right)_{x^{\prime} \rightarrow x} g^{(0) \mu^{\prime} \nu}\right)\right\}\right] .
\end{aligned}
$$

Here, $T_{\mu \nu}^{(1)}$ is a Lagrange multiplier field to impose the update condition. This completes the first renormalization group transformation.

Based on the above expression, we can estimate the partition function after the $(f-1)$ th renormalization group transformation, given by

$$
Z=\int D \phi_{\alpha} \Pi_{k=0}^{f} D \varphi^{(k)} D g_{\mu \nu}^{(k)} D T_{\mu \nu}^{(k)} \exp \left\{-S_{\Lambda}-S_{\mathrm{UV}}-S_{\mathrm{IR}}-S_{\mathrm{Bulk}}\right\} .
$$

Here, $S_{\Lambda}$ is an effective action which is UV-divergent, and given by

$$
\begin{aligned}
S_{\Lambda}= & -\frac{N}{2} \sum_{k=1}^{f} \operatorname{tr}_{x x^{\prime}} \ln (2 d z)-\frac{1}{2} \sum_{k=1}^{f} \operatorname{tr}_{x x^{\prime}} \ln (2 d z) \\
& +\frac{1}{2} \sum_{k=1}^{f} \operatorname{tr}_{x x^{\prime}} \ln \frac{N}{2 u}+\frac{N}{2} \sum_{k=1}^{f} \operatorname{tr}_{x x^{\prime}} \ln \sqrt{g^{(k-1)}} m^{2} .
\end{aligned}
$$

$S_{\mathrm{UV}}$ is an effective action imposing UV boundary conditions for both metric and dual scalar fields, given by

$$
S_{\mathrm{UV}}=N \int d^{D} x\left\{-\frac{1}{2 u} \sqrt{g^{(0)}}\left(\varphi^{(0)}-\xi R^{(0)}\right)^{2}-\sqrt{g^{(0)}} T_{\mu \nu}^{(0)}\left(g^{(0) \mu \nu}-g_{B}^{\mu \nu}\right)\right\}
$$

$S_{\text {IR }}$ is an effective action implementing IR boundary conditions of both metric and dual scalar fields, given by

$$
S_{\mathrm{IR}}=\int d^{D} x \sqrt{g^{(f)}}\left\{g^{(f) \mu \nu}\left(\partial_{\mu} \phi_{\alpha}\right)\left(\partial_{\nu} \phi_{\alpha}\right)+\left(m^{2}+\varphi^{(f)}\right) \phi_{\alpha}^{2}\right\} .
$$

$S_{\text {Bulk }}$ is an effective action to govern the dynamics of both metric and dual scalar fields, given by

$$
\begin{aligned}
S_{\text {Bulk }}= & N(2 d z) \sum_{k=1}^{f} \int d^{D} x \sqrt{g^{(k-1)}}\left\{-\frac{1}{2 u}\left(\frac{\varphi^{(k)}-\varphi^{(k-1)}}{2 d z}\right)^{2}\right. \\
& -T_{\mu \nu}^{(k)}\left(\frac{g^{(k) \mu \nu}-g^{(k-1) \mu \nu}}{2 d z}-g^{(k-1) \mu \nu^{\prime}}\left(\partial_{\nu^{\prime}} \partial_{\mu^{\prime}} G_{x x^{\prime}}^{(k-1)}\right)_{x^{\prime} \rightarrow x} g^{(k-1) \mu^{\prime} \nu}\right) \\
& \left.-\mathcal{C}_{\Lambda}+\mathcal{C}_{R} R^{(k-1)}-\frac{\mathcal{C}_{\varphi}}{2} g^{\mu \nu(k-1)}\left(\partial_{\mu} \varphi^{(k)}\right)\left(\partial_{\nu} \varphi^{(k)}\right)-\mathcal{C}_{\xi} R^{(k-1)} \varphi^{(k) 2}\right\} .
\end{aligned}
$$

$G_{x x^{\prime}}^{(k-1)}$ is the Green's function for the $k$-th renormalization group transformation, given by

$$
\left\{-\frac{1}{\sqrt{g_{x}^{(k-1)}}} \partial_{\mu}\left(\sqrt{g_{x}^{(k-1)}} g_{x}^{\mu \nu(k-1)} \partial_{\nu}\right)+\frac{1}{2 d z}\left(m^{2}+\varphi_{x}^{(k)}\right)\right\} G_{x x^{\prime}}^{(k-1)}=\frac{1}{\sqrt{g_{x}^{(k-1)}}} \delta^{(D)}\left(x-x^{\prime}\right) .
$$


Here, we replaced $\varphi^{(k)}$ with $i \varphi^{(k)}$ and adopt gauge fixing for both uniform speeds of coarse graining as $\alpha^{(k)}=\beta^{(k)}=1$. More discussions on this gauge fixing will be presented below.

The last step is to rewrite the above partition function in the continuous coordinate representation instead of the discrete variable $k$. Considering

$$
\begin{gathered}
(2 d z) \sum_{k=1}^{f} \int d^{D} x \sqrt{g^{(k-1)}} T_{\mu \nu}^{(k)}\left(\frac{g^{(k) \mu \nu}-g^{(k-1) \mu \nu}}{2 d z}\right) \\
\Longrightarrow \int_{0}^{z_{f}} d z \int d^{D} x \sqrt{g(x, z)} T_{\mu \nu}(x, z) \partial_{z} g^{\mu \nu}(x, z)
\end{gathered}
$$

with $(2 d z) \sum_{k=1}^{f} \Longrightarrow \int_{0}^{z_{f}} d z$ and $\frac{g^{(k) \mu \nu}-g^{(k-1) \mu \nu}}{2 d z} \Longrightarrow \partial_{z} g^{\mu \nu}(x, z)$, we obtain

$$
\begin{aligned}
Z= & Z_{\Lambda} \int D \phi_{\alpha}(x) D \varphi(x, z) D g_{\mu \nu}(x, z) D T_{\mu \nu}(x, z) \exp [ \\
& -\int d^{D} x \sqrt{g\left(x, z_{f}\right)}\left\{g^{\mu \nu}\left(x, z_{f}\right)\left[\partial_{\mu} \phi_{\alpha}(x)\right]\left[\partial_{\nu} \phi_{\alpha}(x)\right]+\left[m^{2}+\varphi\left(x, z_{f}\right)\right] \phi_{\alpha}^{2}(x)\right\} \\
& -N \int d^{D} x \sqrt{g(x, 0)}\left\{-\frac{1}{2 u}(\varphi(x, 0)-\xi R(x, 0))^{2}-T_{\mu \nu}(x, 0)\left(g^{\mu \nu}(x, 0)-g_{B}^{\mu \nu}(x)\right)\right\} \\
& -N \int_{0}^{z_{f}} d z \int d^{D} x \sqrt{g(x, z)}\{ \\
& -\frac{1}{2 u}\left[\partial_{z} \varphi(x, z)\right]^{2}-\frac{\mathcal{C}_{\varphi}}{2} g^{\mu \nu}(x, z)\left[\partial_{\mu} \varphi(x, z)\right]\left[\partial_{\nu} \varphi(x, z)\right] \\
& -\mathcal{C}_{\xi} R(x, z)[\varphi(x, z)]^{2}+\frac{1}{2 \kappa}(R(x, z)-2 \Lambda) \\
& \left.\left.-T_{\mu \nu}(x, z)\left(\partial_{z} g^{\mu \nu}(x, z)-g^{\mu \nu^{\prime}}(x, z)\left(\partial_{\nu^{\prime}} \partial_{\mu^{\prime}} G_{x x^{\prime}}\left[g_{\mu \nu}(x, z), \varphi(x, z)\right]\right)_{x^{\prime} \rightarrow x} g^{\mu^{\prime} \nu}(x, z)\right)\right\}\right],
\end{aligned}
$$

where all UV divergent terms are absorbed into a normalization constant $Z_{\Lambda}$. For the Einstein-Hilbert action, we took the following replacements

$$
\mathcal{C}_{R} \equiv \frac{1}{2 \kappa}, \quad \frac{\mathcal{C}_{\Lambda}}{\mathcal{C}_{R}} \equiv 2 \Lambda .
$$

All the dynamical information is encoded into the Green's function, given by

$$
\begin{aligned}
& \left\{-\frac{1}{\sqrt{g(x, z)}} \partial_{\mu}\left(\sqrt{g(x, z)} g^{\mu \nu}(x, z) \partial_{\nu}\right)+\frac{1}{2 d z}\left[m^{2}+\varphi(x, z)\right]\right\} G_{x x^{\prime}}\left[g_{\mu \nu}(x, z), \varphi(x, z)\right] \\
& =\frac{1}{\sqrt{g(x, z)}} \delta^{(D)}\left(x-x^{\prime}\right) .
\end{aligned}
$$

As discussed earlier in section 2, the physical description is quite clear in this effective action. The $D$-dimensional metric tensor evolves through the extra dimension, following the equation of motion

$$
\partial_{z} g^{\mu \nu}(x, z)=g^{\mu \nu^{\prime}}(x, z)\left(\partial_{\nu^{\prime}} \partial_{\mu^{\prime}} G_{x x^{\prime}}\left[g_{\mu \nu}(x, z), \varphi(x, z)\right]\right)_{x^{\prime} \rightarrow x} g^{\mu^{\prime} \nu}(x, z) .
$$

As a result, a fully renormalized metric $g^{\mu \nu}\left(x, z_{f}\right)$ appears in the IR effective action $\mathcal{S}_{\mathrm{IR}}=\int d^{D} x \sqrt{g\left(x, z_{f}\right)}\left\{g^{\mu \nu}\left(x, z_{f}\right)\left[\partial_{\mu} \phi_{\alpha}(x)\right]\left[\partial_{\nu} \phi_{\alpha}(x)\right]+\left[m^{2}+\varphi\left(x, z_{f}\right)\right] \phi_{\alpha}^{2}(x)\right\}$, describing 
all possible renormalizations such as field renormalization, mass renormalization, and interaction renormalization. This evolution equation plays essentially the same role as renormalization group $\beta$-functions.

\subsection{Remarks on the renormalization group transformation for the metric ten- sor}

Although the renormalization group flow of the metric tensor results from quantum fluctuations of matter fields, the metric tensor is not fully dynamical in contrast with the holographic duality conjecture [2-8]. This should be contrasted with the order-parameter field which is promoted to be dynamical in the extra-dimensional space. We recall that the dynamics of the order-parameter field originates from effective self-interactions between matter fields. In this respect we introduce effective tensor-field interactions into the partition function as follows

$$
\begin{aligned}
Z= & \int D \phi_{\alpha} \exp \left[-\int d^{D} x \sqrt{g_{B}}\left\{g_{B}^{\mu \nu}\left(\partial_{\mu} \phi_{\alpha}\right)\left(\partial_{\nu} \phi_{\alpha}\right)+m^{2} \phi_{\alpha}^{2}+\xi R_{B} \phi_{\alpha}^{2}\right.\right. \\
& \left.\left.+\frac{u}{2 N} \phi_{\alpha}^{2} \phi_{\beta}^{2}+\frac{\lambda}{2 N}\left[\left(\partial^{\mu} \phi_{\alpha}\right)\left(\partial^{\nu} \phi_{\alpha}\right)\right]\left[\left(\partial_{\mu} \phi_{\beta}\right)\left(\partial_{\nu} \phi_{\beta}\right)\right]\right\}\right] .
\end{aligned}
$$

It is natural to expect that the effective interaction term $\frac{\lambda}{2 N}\left[\left(\partial^{\mu} \phi_{\alpha}\right)\left(\partial^{\nu} \phi_{\alpha}\right)\right]\left[\left(\partial_{\mu} \phi_{\beta}\right)\left(\partial_{\nu} \phi_{\beta}\right)\right]$ is irrelevant in the renormalization group sense as long as the coupling constant $\lambda$ remains to be below a critical value. However, we point out that these tensor-type quantum fluctuations promote the emergent metric tensor to be fully dynamical [41]. Although one can take into account an effective interaction term of the exact energy-momentum tensor in the effective Lagrangian, the above introduction of the effective interaction term is sufficient in discussing which approximation scheme has to be used for the metric renormalization-group transformation.

Performing the Hubbard-Stratonovich transformation for both effective interactions, we obtain

$$
\begin{aligned}
Z= & \int D \phi_{\alpha} D \varphi^{(0)} D g_{\mu \nu}^{(0)} D t_{\mu \nu}^{(0)} D g_{\mu \nu} \exp \left[-\int d^{D} x \sqrt{g^{(0)}}\left\{\left(g^{\mu \nu(0)}-i g^{\mu \nu}\right)\left(\partial_{\mu} \phi_{\alpha}\right)\left(\partial_{\nu} \phi_{\alpha}\right)\right.\right. \\
& \left.\left.+\left(m^{2}-i \varphi^{(0)}\right) \phi_{\alpha}^{2}+\xi R^{(0)} \phi_{\alpha}^{2}+\frac{N}{2 u} \varphi^{(0) 2}+\frac{N}{2 \lambda} g^{\mu \nu} g_{\mu \nu}-N t_{\mu \nu}^{(0)}\left(g^{\mu \nu(0)}-g_{B}^{\mu \nu}\right)\right\}\right], \quad(3
\end{aligned}
$$

where $t_{\mu \nu}^{(0)}$ is a Lagrange multiplier field to impose the initial condition $g^{\mu \nu(0)}=g_{B}^{\mu \nu}$. Shifting the metric tensor in the following way

$$
g^{\mu \nu(0)} \Longrightarrow g^{\mu \nu(0)}+i g^{\mu \nu}
$$

we obtain

$$
\begin{aligned}
Z= & \int D \phi_{\alpha} D \varphi^{(0)} D g_{\mu \nu}^{(0)} D t_{\mu \nu}^{(0)} D g_{\mu \nu} \exp \left[-\int d^{D} x \sqrt{\operatorname{det}\left[g_{\mu \nu}^{(0)}+i g_{\mu \nu}\right]}\left\{g^{\mu \nu(0)}\left(\partial_{\mu} \phi_{\alpha}\right)\left(\partial_{\nu} \phi_{\alpha}\right)\right.\right. \\
& +\left(m^{2}-i \varphi^{(0)}\right) \phi_{\alpha}^{2}+\xi R\left[g_{\mu \nu}^{(0)}+i g_{\mu \nu}\right] \phi_{\alpha}^{2} \\
& \left.\left.+\frac{N}{2 u} \varphi^{(0) 2}+\frac{N}{2 \lambda} g^{\mu \nu} g_{\mu \nu}-N t_{\mu \nu}^{(0)}\left(g^{\mu \nu(0)}+i g^{\mu \nu}-g_{B}^{\mu \nu}\right)\right\}\right] .
\end{aligned}
$$


We now perform the path integral $\int D t_{\mu \nu}^{(0)} D g_{\mu \nu}$. The approximation that we have to use is to keep quantum fluctuations of the metric tensor up to the linear order. In other words, we neglect the $i g_{\mu \nu}$ contribution in both the determinant and Ricci scalar. As a result, we obtain

$$
\begin{aligned}
Z \approx & \int D \phi_{\alpha} D \varphi^{(0)} D g_{\mu \nu}^{(0)} \exp \left[-\int d^{D} x \sqrt{g^{(0)}}\left\{g^{\mu \nu(0)}\left(\partial_{\mu} \phi_{\alpha}\right)\left(\partial_{\nu} \phi_{\alpha}\right)+\left(m^{2}-i \varphi^{(0)}\right) \phi_{\alpha}^{2}+\xi R^{(0)} \phi_{\alpha}^{2}\right.\right. \\
& \left.\left.+\frac{N}{2 u} \varphi^{(0) 2}-\frac{N}{2 \lambda}\left(g^{\mu \nu(0)}-g_{B}^{\mu \nu}\right)\left(g_{\mu \nu}^{(0)}-g_{B \mu \nu}\right)\right\}\right] .
\end{aligned}
$$

To perform the renormalization group transformation for the metric tensor, we introduce an auxiliary field in the following way

$$
\begin{aligned}
Z= & \int D \phi_{\alpha} D \varphi^{(0)} D \varphi^{(1)} D g_{\mu \nu}^{(0)} D G_{\mu \nu}^{(0)} \\
& \exp \left[-\int d^{D} x \sqrt{i^{-1} \operatorname{det}\left[g_{\mu \nu}^{(0)}+g_{B \mu \nu}\right]}\left\{i\left[g^{\mu \nu(0)}+g_{B}^{\mu \nu}\right]\left(\partial_{\mu} \phi_{\alpha}\right)\left(\partial_{\nu} \phi_{\alpha}\right)+\left(m^{2}-i e^{-\beta^{(0)} d z} \varphi^{(1)}\right) \phi_{\alpha}^{2}\right.\right. \\
& +\frac{N}{2 u}\left(\varphi^{(0)}-i e^{-\beta^{(0)} d z} \xi R\left[i\left(g_{\mu \nu}^{(0)}+g_{B \mu \nu}\right)\right]\right)^{2}+\frac{N}{2 u} \frac{1}{e^{2 \beta^{(0)}} d z-1}\left(\varphi^{(1)}-\varphi^{(0)}\right)^{2} \\
& \left.\left.+\frac{N}{2 \lambda} g^{\mu \nu(0)} g_{\mu \nu}^{(0)}+\frac{N}{2 \lambda_{G}} G^{\mu \nu(0)} G_{\mu \nu}^{(0)}\right\}\right]
\end{aligned}
$$

where the renormalization group transformation for the order-parameter field has been performed and $g^{\mu \nu(0)} \Longrightarrow i\left(g^{\mu \nu(0)}+g_{B}^{\mu \nu}\right)$ has been taken. Separating slow and fast degrees of freedom in the metric tensor as

$$
g^{\mu \nu(0)} \Longrightarrow g^{\mu \nu(0)}+\mathcal{G}^{\mu \nu(0)}, \quad G^{\mu \nu(0)} \Longrightarrow c_{g}^{(0)} g^{\mu \nu(0)}+c_{G}^{(0)} \mathcal{G}^{\mu \nu(0)},
$$

where both coefficients are given by

$$
c_{g}^{(0)}=\frac{\lambda^{-1}}{\mu^{(0)} \lambda_{G}^{-1 / 2}}, \quad c_{G}^{(0)}=-\frac{\mu^{(0)}}{\lambda_{G}^{-1 / 2}}, \quad \mu^{(0)}=\frac{\lambda^{-1 / 2}}{\sqrt{e^{2 \delta^{(0)}} d z-1}},
$$

and rescaling both degrees of freedom as

$$
g^{\mu \nu(0)} \Longrightarrow e^{-\delta^{(0)} d z} g^{\mu \nu(0)}, \quad \mathcal{G}^{\mu \nu(0)} \Longrightarrow e^{-\delta^{(0)} d z} \mathcal{G}^{\mu \nu(0)},
$$

where $\delta^{(0)}$ is the local speed of coarse graining, we have

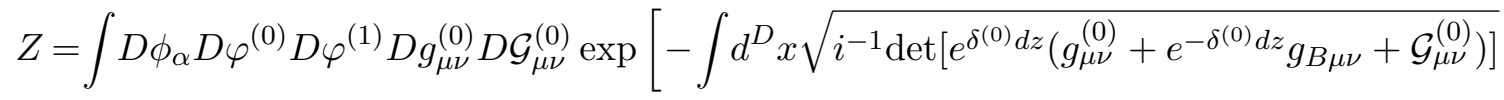

$$
\begin{aligned}
& \left\{i e^{-\delta^{(0)} d z}\left(g^{\mu \nu(0)}+e^{\delta^{(0)}} d z g_{B}^{\mu \nu}+\mathcal{G}^{\mu \nu(0)}\right)\left(\partial_{\mu} \phi_{\alpha}\right)\left(\partial_{\nu} \phi_{\alpha}\right)+\left(m^{2}-i e^{-\beta^{(0)}} d z \varphi^{(1)}\right) \phi_{\alpha}^{2}\right. \\
& +\frac{N}{2 u}\left(\varphi^{(0)}-i e^{-\beta^{(0)} d z} \xi R\left[i e^{\delta^{(0)} d z}\left(g_{\mu \nu}^{(0)}+e^{-\delta^{(0)} d z} g_{B \mu \nu}+\mathcal{G}_{\mu \nu}^{(0)}\right)\right]\right)^{2} \\
& \left.\left.+\frac{N}{2 u} \frac{1}{e^{2 \beta^{(0)} d z}-1}\left(\varphi^{(1)}-\varphi^{(0)}\right)^{2}+\frac{N}{2 \lambda} g^{\mu \nu(0)} g_{\mu \nu}^{(0)}+\frac{N}{2 \lambda} \frac{1}{e^{2 \delta^{(0)}} d z-1} \mathcal{G}^{\mu \nu(0)} \mathcal{G}_{\mu \nu}^{(0)}\right\}\right] .
\end{aligned}
$$


To perform the renormalization group transformation for the metric tensor, we consider the linear approximation again for the $\mathcal{G}_{\mu \nu}^{(0)}$ path integral, where the $\mathcal{G}_{\mu \nu}^{(0)}$ contribution in both the determinant and the Ricci scalar is neglected. As a result, we obtain

$$
\begin{aligned}
Z= & \int D \phi_{\alpha} D \varphi^{(0)} D \varphi^{(1)} D g_{\mu \nu}^{(0)} \exp \left[-\int d^{D} x \sqrt{e^{\delta^{(0)}} d z} g^{(0)}\left\{e^{-\delta^{(0)} d z} g^{\mu \nu(0)}\left(\partial_{\mu} \phi_{\alpha}\right)\left(\partial_{\nu} \phi_{\alpha}\right)\right.\right. \\
& +\left(m^{2}-i e^{-\beta^{(0)}} d z \varphi^{(1)}\right) \phi_{\alpha}^{2}+\frac{N}{2 u}\left(\varphi^{(0)}-i e^{-\beta^{(0)}} d z \xi R^{(0)}\right)^{2}+\frac{N}{2 u} \frac{1}{e^{2 \beta^{(0)}} d z-1}\left(\varphi^{(1)}-\varphi^{(0)}\right)^{2} \\
& -\frac{N}{2 \lambda}\left(g^{\mu \nu(0)}-e^{\delta^{(0)} d z} g_{B}^{\mu \nu}\right)\left(g_{\mu \nu}^{(0)}-e^{-\delta^{(0)} d z} g_{B \mu \nu}\right) \\
& \left.\left.+\frac{\lambda}{2 N}\left[1-e^{-2 \delta^{(0)} d z}\right]\left(\partial^{\mu} \phi_{\alpha}\right)\left(\partial^{\nu} \phi_{\alpha}\right)\left(\partial_{\mu} \phi_{\beta}\right)\left(\partial_{\nu} \phi_{\beta}\right)\right\}\right],
\end{aligned}
$$

where an effective interaction term $\frac{\lambda}{2 N}\left[1-e^{-2 \delta^{(0)}} d z\right]\left(\partial^{\mu} \phi_{\alpha}\right)\left(\partial^{\nu} \phi_{\alpha}\right)\left(\partial_{\mu} \phi_{\beta}\right)\left(\partial_{\nu} \phi_{\beta}\right)$ has been generated. Performing the Hubbard-Stratonovich transformation for the newly generated effective interaction term and updating the metric tensor in the boundary action, we obtain

$$
\begin{aligned}
Z= & \int D \phi_{\alpha} D \varphi^{(0)} D \varphi^{(1)} D g_{\mu \nu}^{(0)} D \tilde{g}_{\mu \nu}^{(0)} \exp \left[-\int d^{D} x \sqrt{e^{\delta^{(0)}} d z \tilde{g}^{(0)}}\left\{e^{-\delta^{(0)} d z} \tilde{g}^{\mu \nu(0)}\left(\partial_{\mu} \phi_{\alpha}\right)\left(\partial_{\nu} \phi_{\alpha}\right)\right.\right. \\
& +\left(m^{2}-i e^{-\beta^{(0)} d z} \varphi^{(1)}\right) \phi_{\alpha}^{2}+\frac{N}{2 u} \frac{1}{e^{2 \beta^{(0)}} d z-1}\left(\varphi^{(1)}-\varphi^{(0)}\right)^{2} \\
& \left.-\frac{N}{2 \lambda} \frac{1}{e^{2 \delta^{(0)} d z}-1}\left(\tilde{g}^{\mu \nu(0)}-g^{\mu \nu(0)}\right)\left(\tilde{g}_{\mu \nu}^{(0)}-g_{\mu \nu}^{(0)}\right)\right\} \\
& -\int d^{D} x \sqrt{e^{\delta^{(0)}} d z} g^{(0)}\left\{\frac{N}{2 u}\left(\varphi^{(0)}-i e^{-\beta^{(0)} d z} \xi R^{(0)}\right)^{2}\right. \\
& \left.\left.-\frac{N}{2 \lambda}\left(g^{\mu \nu(0)}-e^{\delta^{(0)} d z} g_{B}^{\mu \nu}\right)\left(g_{\mu \nu}^{(0)}-e^{-\delta^{(0)} d z} g_{B \mu \nu}\right)\right\}\right],
\end{aligned}
$$

where $\tilde{g}^{\mu \nu(0)}$ is an updated metric tensor.

Finally, we perform the renormalization group transformation for the matter sector as discussed in the previous section. Taking the locality approximation for the Green's function and keeping all terms up to the linear order in $d z$, we find

$$
\begin{aligned}
Z= & \int D \phi_{\alpha} D \varphi^{(0)} D \varphi^{(1)} D g_{\mu \nu}^{(0)} D \tilde{g}_{\mu \nu}^{(0)} \\
& \exp \left[-\int d^{D} x \sqrt{\tilde{g}^{(0)}}\left\{\left(\tilde{g}^{\mu \nu(0)}-\tilde{g}^{\mu \delta(0)}\left(\partial_{\delta} \partial_{\delta^{\prime}} G_{x x^{\prime}}^{(0)}\right)_{x^{\prime} \rightarrow x} \tilde{g}^{\delta^{\prime} \nu(0)}\right)\left(\partial_{\mu} \phi_{\alpha}\right)\left(\partial_{\nu} \phi_{\alpha}\right)\right.\right. \\
& +\left(m^{2}-i \varphi^{(1)}\right) \phi_{\alpha}^{2}+2 \beta^{(0)} d z \frac{N}{2 u}\left(\frac{\varphi^{(1)}-\varphi^{(0)}}{2 \beta^{(0)} d z}\right)^{2}-2 \delta^{(0)} d z \frac{N}{2 \lambda}\left(\frac{\tilde{g}^{\mu \nu(0)}-g^{\mu \nu(0)}}{2 \delta^{(0)} d z}\right)\left(\frac{\tilde{g}_{\mu \nu}^{(0)}-g_{\mu \nu}^{(0)}}{2 \delta^{(0)} d z}\right) \\
& \left.+2 \alpha^{(0)} d z N\left(-\mathcal{C}_{\Lambda}+\mathcal{C}_{R} \tilde{R}^{(0)}+\frac{\mathcal{C}_{\varphi}}{2} \tilde{g}^{\mu \nu(0)}\left(\partial_{\mu} \varphi^{(1)}\right)\left(\partial_{\nu} \varphi^{(1)}\right)+\mathcal{C}_{\xi} \tilde{R}^{(0)} \varphi^{(1) 2}\right)\right\} \\
& \left.-\int d^{D} x \sqrt{g^{(0)}}\left\{\frac{N}{2 u}\left(\varphi^{(0)}-i \xi R^{(0)}\right)^{2}-\frac{N}{2 \lambda}\left(g^{\mu \nu(0)}-g_{B}^{\mu \nu}\right)\left(g_{\mu \nu}^{(0)}-g_{B \mu \nu}\right)\right\}\right] .
\end{aligned}
$$

Upgrading the metric tensor $\tilde{g}_{\mu \nu}^{(0)}$ to make the kinetic-energy term be invariant, we rewrite 
the above expression as follows

$$
\begin{aligned}
Z= & \int D \phi_{\alpha} D \varphi^{(0)} D \varphi^{(1)} D g_{\mu \nu}^{(0)} D g_{\mu \nu}^{(1)} \\
& \exp \left[-\int d^{D} x \sqrt{g^{(1)}}\left\{g^{\mu \nu(1)}\left(\partial_{\mu} \phi_{\alpha}\right)\left(\partial_{\nu} \phi_{\alpha}\right)+\left(m^{2}-i \varphi^{(1)}\right) \phi_{\alpha}^{2}\right.\right. \\
& +2 \beta^{(0)} d z \frac{N}{2 u}\left(\frac{\varphi^{(1)}-\varphi^{(0)}}{2 \beta^{(0)} d z}\right)^{2}-2 \delta^{(0)} d z \frac{N}{2 \lambda}\left(\frac{g^{\mu \nu(1)}-g^{\mu \nu(0)}}{2 \delta^{(0)} d z}-g^{\mu \delta(0)}\left(\partial_{\delta} \partial_{\delta^{\prime}} G_{x x^{\prime}}^{(0)}\right)_{x^{\prime} \rightarrow x} g^{\delta^{\prime} \nu(0)}\right) \\
& \times\left(\frac{g_{\mu \nu}^{(1)}-g_{\mu \nu}^{(0)}}{2 \delta^{(0)} d z}-g_{\mu \delta}^{(0)}\left(\partial^{\delta} \partial^{\delta^{\prime}} G_{x x^{\prime}}^{(0)}\right)_{x^{\prime} \rightarrow x} g_{\delta^{\prime} \nu}^{(0)}\right) \\
& \left.+2 \alpha^{(0)} d z N\left(-\mathcal{C}_{\Lambda}+\mathcal{C}_{R} R^{(1)}+\frac{\mathcal{C}_{\varphi}}{2} g^{\mu \nu(1)}\left(\partial_{\mu} \varphi^{(1)}\right)\left(\partial_{\nu} \varphi^{(1)}\right)+\mathcal{C}_{\xi} R^{(1)} \varphi^{(1) 2}\right)\right\} \\
& \left.-\int d^{D} x \sqrt{g^{(0)}}\left\{\frac{N}{2 u}\left(\varphi^{(0)}-i \xi R^{(0)}\right)^{2}-\frac{N}{2 \lambda}\left(g^{\mu \nu(0)}-g_{B}^{\mu \nu}\right)\left(g_{\mu \nu}^{(0)}-g_{B \mu \nu}\right)\right\}\right]
\end{aligned}
$$

Repeating all these renormalization group transformations within the recursion framework and rewriting the recursive expression in the continuous variable $z$, we obtain

$$
\begin{aligned}
Z= & Z_{\Lambda} \int D \phi_{\alpha}(x) D \varphi(x, z) D g_{\mu \nu}(x, z) \exp [ \\
& -\int d^{D} x \sqrt{g\left(x, z_{f}\right)}\left\{g^{\mu \nu}\left(x, z_{f}\right)\left[\partial_{\mu} \phi_{\alpha}(x)\right]\left[\partial_{\nu} \phi_{\alpha}(x)\right]+\left[m^{2}+\varphi\left(x, z_{f}\right)\right] \phi_{\alpha}^{2}(x)\right\} \\
& -N \int d^{D} x \sqrt{g(x, 0)}\left\{-\frac{1}{2 u}(\varphi(x, 0)-\xi R(x, 0))^{2}-\frac{1}{2 \lambda}\left(g^{\mu \nu}(x, 0)-g_{B}^{\mu \nu}(x)\right)^{2}\right\} \\
& -N \int_{0}^{z_{f}} d z \int d^{D} x \sqrt{g(x, z)}\{ \\
& -\frac{1}{2 u}\left[\partial_{z} \varphi(x, z)\right]^{2}-\frac{\mathcal{C}_{\varphi}}{2} g^{\mu \nu}(x, z)\left[\partial_{\mu} \varphi(x, z)\right]\left[\partial_{\nu} \varphi(x, z)\right]-\mathcal{C}_{\xi} R(x, z)[\varphi(x, z)]^{2} \\
& -\frac{1}{2 \lambda}\left(\partial_{z} g^{\mu \nu}(x, z)-g^{\mu \nu^{\prime}}(x, z)\left(\partial_{\nu^{\prime}} \partial_{\mu^{\prime}} G_{x x^{\prime}}\left[g_{\mu \nu}(x, z), \varphi(x, z)\right]\right)_{x^{\prime} \rightarrow x^{\prime}} g^{\mu^{\prime} \nu}(x, z)\right)^{2} \\
& \left.\left.+\frac{1}{2 \kappa}(R(x, z)-2 \Lambda)\right\}\right] .
\end{aligned}
$$

Here, the second derivative with respect to $z$ coordinatizing the extra dimension arises in the metric evolution, resulting from essentially the same procedure as that in the orderparameter evolution $\left[\partial_{z} \varphi(x, z)\right]^{2}$, where the linear approximation for the metric tensor has been used. It is interesting to notice that the linear approximation for quantum fluctuations of the metric tensor gives rise to the highly nonlinear renormalization group flow, shown in eq. (3.57), where the metric-tensor renormalization is intertwined with the renormalization of the order-parameter field through the Green's function of the matter field. In this case renormalization group $\beta$-functions are given by IR boundary conditions, where the linear derivative in $z$ appears from the bulk effective action by the technique of integration-byparts. In the next section we demonstrate that the evolution equations of the metric tensor along the extra dimensional space are nothing but the renormalization group $\beta$-functions.

Before going further, we point out that this holographic dual effective field theory is not covariant but a gauge-fixed version. In other words, the metric-tensor components involved 
with the extra dimension are gauge-fixed as follows: $g_{D D}(x, z)=1$ and $g_{\mu D}(x, z)=0$ with $\mu=0, \ldots, D-1$. It turns out that the gauge choice of $\alpha^{(k)}=1$ gives rise to $g_{D D}(x, z)=1$. On the other hand, the gauge freedom in $g_{\mu D}(x, z)=0$ results from the invariance of the partition function with respect to $D$-dimensional diffeomorphism after the renormalization group transformation with $d z$ [31-33]. While we do not delve into this issue here, a fully covariant formulation has been constructed in the absence of dual scalar fields, where the $D$-dimensional Einstein-Hilbert action is uplifted into the $(D+1)$-dimensional EinsteinHilbert one via recursive renormalization group transformations [31-33].

We also point out that the order of the renormalization group transformations for the dual and original boson fields is immaterial. We recall that the $d z \rightarrow 0$ limit with $z_{f} \equiv$ $d z \sum_{k=1}^{f}=f d z$ controls our recursive renormalization group transformations. The order of the renormalization group transformations does not matter in this controllable limit.

\section{Physical interpretation of the emergent dynamical metric tensor: from renormalization group equations of coupling parameters to the evolu- tion equation of the metric tensor}

In this section we demonstrate explicitly that renormalization group $\beta$-functions in the quantum field theory are nothing but IR boundary conditions in the large $N$ limit of the holographic dual field theory. We do so by comparing our holographic theory and yet another real space renormalization group transformation (the Kadanoff block-spin transformation). For simplicity in the presentation, we switch off the self-interactions and consider a one-dimensional lattice field theory, given by

$$
Z=\int D \Phi_{i} \exp \left[-\int_{0}^{\beta} d \tau \sum_{i=1}^{M}\left\{\left(\partial_{\tau} \Phi_{i}\right)^{2}-t\left(\Phi_{i} \Phi_{i+1}+\Phi_{i+1} \Phi_{i}\right)+m^{2} \Phi_{i}^{2}\right\}\right] .
$$

Here, $\Phi_{i}$ is a real scalar field at site $i . t$ is a hopping integral and $m$ is a mass parameter. In the case of one dimensional lattice, it is straightforward to perform the Kadanoff blockspin transformation [71]. Integrating out all dynamical fields at even lattice sites, we obtain an effective field theory for odd-site scalar fields with renormalized hopping and mass parameters. Implementing this renormalization group transformation in a recursive way [48], we obtain the following expression of the partition function

$$
\begin{aligned}
Z= & \int D \Phi(i, \tau) D t(z) D m^{2}(z) \\
& \times \delta(t(0)-t) \delta\left(m^{2}(0)-m^{2}\right) \delta\left(\partial_{z} m^{2}(z)+\frac{[t(z)]^{2}}{a m^{2}(z)}\right) \delta\left(\partial_{z} t(z)+\frac{1}{a} t(z)-\frac{[t(z)]^{2}}{2 a m^{2}(z)}\right) \\
& \times \exp \left[-\int_{0}^{\beta} d \tau \sum_{i=1}^{M}\left\{\left(\partial_{\tau} \Phi(i, \tau)\right)^{2}+m^{2}\left(z_{f}\right)[\Phi(i, \tau)]^{2}\right.\right. \\
& \left.\left.-t\left(z_{f}\right)(\Phi(i, \tau) \Phi(i+1, \tau)+\Phi(i+1, \tau) \Phi(i, \tau))\right\}-\frac{M}{4 a} \int_{0}^{z_{f}} d z \sum_{i \omega_{n}} \ln \left(\omega_{n}^{2}+m^{2}(z)\right)\right] .
\end{aligned}
$$


Here, $a$ is a scale for the renormalization group transformation, defined in eqs. (A.8) and (A.9), which corresponds to $d z$ in the previous section. All details are shown in appendix A, where recursive Kadanoff block-spin transformations have been performed in the presence of self-interactions. We point out that both the hopping and mass parameters are renormalized to appear in the IR boundary action. In this respect two $\delta$-function constraints serve as renormalization group $\beta$-functions for the hopping and mass parameters, respectively.

To show the equivalence between the above renormalization group $\beta$-functions and the evolution equations for the metric-tensor fields, we recall the gravity formulation, given by

$$
\begin{aligned}
Z= & \int D \phi_{\alpha}(x) D g_{\mu \nu}(x, z) \delta\left(g^{\mu \nu}(x, 0)-g_{B}^{\mu \nu}\right) \\
& \times \delta\left(\partial_{z} g^{\mu \nu}(x, z)-g^{\mu \nu^{\prime}}(x, z)\left(\partial_{\nu^{\prime}} \partial_{\mu^{\prime}} G_{x x^{\prime}}\left[g_{\mu \nu}(x, z)\right]\right)_{x^{\prime} \rightarrow x} g^{\mu^{\prime} \nu}(x, z)\right) \\
& \times \exp \left[-\int d^{D} x \sqrt{g\left(x, z_{f}\right)}\left\{g^{\mu \nu}\left(x, z_{f}\right)\left(\partial_{\mu} \phi_{\alpha}(x)\right)\left(\partial_{\nu} \phi_{\alpha}(x)\right)+m^{2} \phi_{\alpha}^{2}(x)\right\}\right. \\
& \left.-\frac{N}{2 \kappa} \int_{0}^{z_{f}} d z \int d^{D} x \sqrt{g(x, z)}(R(x, z)-2 \Lambda)\right],
\end{aligned}
$$

which results from the UV theory of

$$
Z=\int D \phi_{\alpha}(x) \exp \left[-\int d^{D} x \sqrt{g_{B}}\left\{g_{B}^{\mu \nu}\left(\partial_{\mu} \phi_{\alpha}\right)\left(\partial_{\nu} \phi_{\alpha}\right)+m^{2} \phi_{\alpha}^{2}\right\}\right] .
$$

Comparing each term between eq. (4.2) and eq. (4.3), we observe the following correspondences,

$$
\sqrt{g\left(x, z_{f}\right)} m^{2}=m^{2}\left(z_{f}\right)-2 t\left(z_{f}\right) \Longrightarrow \frac{1}{\sqrt{g\left(x, z_{f}\right)}}=\frac{m^{2}}{m^{2}\left(z_{f}\right)-2 t\left(z_{f}\right)}
$$

for the metric determinant,

$$
\sqrt{g\left(x, z_{f}\right)} g^{x x}\left(x, z_{f}\right)=t\left(z_{f}\right) \Longrightarrow g^{x x}\left(x, z_{f}\right)=\frac{m^{2}}{m^{2}\left(z_{f}\right)-2 t\left(z_{f}\right)} t\left(z_{f}\right)
$$

for the $g^{x x}\left(x, z_{f}\right)$ component, and

$$
\sqrt{g\left(x, z_{f}\right)} g^{\tau \tau}\left(x, z_{f}\right)=1 \Longrightarrow g^{\tau \tau}\left(x, z_{f}\right)=\frac{m^{2}}{m^{2}\left(z_{f}\right)-2 t\left(z_{f}\right)}
$$

for the $g^{\tau \tau}\left(x, z_{f}\right)$ component. We recall the gauge choice

$$
g^{z z}(x, z)=1
$$

We also point out the correspondence in the Green's function, given by

$$
G\left[q_{0}, q_{x} ; t(z), m(z)\right]=\frac{1}{q_{0}^{2}+t(z) q_{x}^{2}+\frac{m^{2}(z)-2 t(z)}{2 d z}},
$$


where $q_{0}$ and $q_{x}$ are temporal and spatial components of the momentum, respectively. Introducing all these correspondences into the evolution equations for the metric tensors, given by

$$
\partial_{z} g^{\tau \tau}(z)=g^{\tau \tau}(z)\left\{\int d q_{0} d q_{x} \frac{q_{0}^{2}}{q_{0}^{2}+t(z) q_{x}^{2}+\frac{m^{2}(z)-2 t(z)}{2 d z}}\right\} g^{\tau \tau}(z)
$$

and

$$
\partial_{z} g^{x x}(z)=g^{x x}(z)\left\{\int d q_{0} d q_{x} \frac{q_{x}^{2}}{q_{0}^{2}+t(z) q_{x}^{2}+\frac{m^{2}(z)-2 t(z)}{2 d z}}\right\} g^{x x}(z),
$$

we obtain the following equations for both mass and hopping parameters,

$$
\partial_{z} m^{2}(z)-2 \partial_{z} t(z)=-\pi \frac{m^{2}}{2 d z}\left(\int d q_{0} \frac{q_{0}^{2}}{\sqrt{q_{0}^{2}+1}}\right) \frac{m^{2}(z)-2 t(z)}{\sqrt{t(z)}}
$$

and

$$
m^{2}(z) \partial_{z} t(z)-t(z) \partial_{z} m^{2}(z)=\pi \frac{m^{2}}{2 d z}\left(\int d q_{x} \frac{q_{x}^{2}}{\sqrt{q_{x}^{2}+1}}\right) \sqrt{t(z)}\left[m^{2}(z)-2 t(z)\right] .
$$

These two coupled equations are reduced into

$$
\partial_{z} m^{2}(z)=-\pi \frac{m^{2}}{2 d z}\left(\int d q_{0} \frac{q_{0}^{2}}{\sqrt{q_{0}^{2}+1}}\right) \frac{m^{2}(z)-2 t(z)}{\sqrt{t(z)}}
$$

and

$$
\partial_{z} t(z)=0
$$

Redefining the mass parameter as

$$
M^{2}(z) \equiv m^{2}(z)+2 t(z)
$$

we rewrite eq. (4.14) as

$$
\partial_{z} M^{2}(z)=-\pi \frac{m^{2}}{2 d z \sqrt{t}}\left(\int d q_{0} \frac{q_{0}^{2}}{\sqrt{q_{0}^{2}+1}}\right) M^{2}(z) .
$$

To show the equivalence between this equation and the $\beta$-function of the mass parameter, we consider the following fixed point, given by

$$
\partial_{z} t(z)=-\frac{1}{a} t(z)+\frac{[t(z)]^{2}}{2 a m^{2}(z)} \Longrightarrow 0
$$

in the $\beta$-function of the hopping parameter. Then, the $\beta$-function of the mass parameter is

$$
\partial_{z} m^{2}(z)=-\frac{[t(z)]^{2}}{a m^{2}(z)} \Longrightarrow-\frac{4}{a} m^{2}(z)
$$


Comparing eq. (4.17) with eq. (4.19), we obtain the correspondence between the scale $d z$ of the Polchinski real-space renormalization group transformation and the scale $a$ of the Kadanoff block-spin transformation as follows

$$
\frac{2 d z}{a}=\pi \frac{m^{2}}{4 \sqrt{t}}\left(\int d q_{0} \frac{q_{0}^{2}}{\sqrt{q_{0}^{2}+1}}\right) .
$$

This completes our demonstration that the evolution equations of the metric tensor are nothing but the renormalization-group $\beta$-functions of the coupling constants.

\section{Evaluation of entanglement entropy based on the heat kernel method}

\subsection{Entanglement entropy}

As we have demonstrated that the quantum field theory is geometrized in the large $N$ limit, we now ask how quantum entanglement in the quantum field theory is encoded into the classical geometry in the holographic dual reformulation. As discussed before, we address this issue by calculating the entanglement entropy.

Entanglement entropy is a subsystem von Neumann entropy $\mathcal{S}_{E E}=-\operatorname{tr}_{A} \rho_{A} \ln \rho_{A}$, given by a reduced density matrix of the subsystem $\rho_{A}=\operatorname{tr}_{B} \rho_{A \cup B}$, where $\rho_{A \cup B}$ is the density matrix of a total system $A \cup B$. This von Neumann entropy can be obtained from the Renyi entropy $\mathcal{S}_{R E}=\frac{1}{1-n} \operatorname{tr}_{A} \rho_{A}^{n}$ as follows [34-39]

$$
\begin{aligned}
\mathcal{S}_{E E}\left(z_{f}\right) & =\lim _{n \rightarrow 1} \frac{1}{1-n} \operatorname{tr}_{A} \rho_{A}^{n}=\lim _{n \rightarrow 1} \frac{\ln Z_{n}\left(z_{f}\right)-n \ln Z_{1}\left(z_{f}\right)}{1-n} \\
& =-\left[\partial_{n} \ln Z_{n}\left(z_{f}\right)\right]_{n=1}+\ln Z_{1}\left(z_{f}\right) .
\end{aligned}
$$

Here, $Z_{1}\left(z_{f}\right)$ is the partition function of the emergent holographic dual description with an IR cutoff $z_{f}$, defined in the Riemann geometry without a conical singularity and given by the $D$-dimensional metric of $d s^{2}=d r^{2}+r^{2} d \theta^{2}+\delta_{i j} d x_{\perp}^{i} d x_{\perp}^{j}$ with $i \& j=2, \ldots, D-1$. For simplicity, we do not consider the self-interactions in the channel of energy-momentum tensor-currents. Then, the partition function is given by

$$
\begin{aligned}
Z_{1}\left(z_{f}\right)= & \int D \phi_{\alpha}(x) D \varphi(x, z) D g_{\mu \nu}(x, z) \delta\left(g^{\mu \nu}(x, 0)-g_{B}^{\mu \nu}\right) \\
& \times \delta\left(\partial_{z} g^{\mu \nu}(x, z)-g^{\mu \nu^{\prime}}(x, z)\left(\partial_{\nu^{\prime}} \partial_{\mu^{\prime}} G_{x x^{\prime}}\left[g_{\mu \nu}(x, z), \varphi(x, z)\right]\right)_{x^{\prime} \rightarrow x} g^{\mu^{\prime} \nu}(x, z)\right) \\
& \times \exp \left[-\int d^{D} x \sqrt{g\left(x, z_{f}\right)}\left\{g^{\mu \nu}\left(x, z_{f}\right)\left[\partial_{\mu} \phi_{\alpha}(x)\right]\left[\partial_{\nu} \phi_{\alpha}(x)\right]+\left[m^{2}-i \varphi\left(x, z_{f}\right)\right] \phi_{\alpha}^{2}(x)\right\}\right. \\
& -N \int d^{D} x \sqrt{g(x, 0)}\left\{\frac{1}{2 u}(\varphi(x, 0)-i \xi R(x, 0))^{2}\right\} \\
& -N \int_{0}^{z_{f}} d z \int d^{D} x \sqrt{g(x, z)}\{ \\
& +\frac{1}{2 u}\left[\partial_{z} \varphi(x, z)\right]^{2}+\frac{\mathcal{C}_{\varphi}}{2} g^{\mu \nu}(x, z)\left[\partial_{\mu} \varphi(x, z)\right]\left[\partial_{\nu} \varphi(x, z)\right]+\mathcal{C}_{\xi} R(x, z)[\varphi(x, z)]^{2} \\
& \left.\left.+\frac{1}{2 \kappa}(R(x, z)-2 \Lambda)\right\}\right] .
\end{aligned}
$$


On the other hand, $Z_{n}\left(z_{f}\right)$ is the partition function of the emergent holographic dual description with an IR cutoff $z_{f}$, defined in the Riemann geometry with a conical singularity and given by the $D$-dimensional metric of $d s^{2}=d r^{2}+n^{2} r^{2} d \theta^{2}+\delta_{i j} d x_{\perp}^{i} d x_{\perp}^{j}$ with $i \& j=$ $2, \ldots, D-1[47]$. It is given by

$$
\begin{aligned}
Z_{n}\left(z_{f}\right)= & \int D \phi_{\alpha, n}(x) D \varphi_{n}(x, z) D g_{\mu \nu, n}(x, z) \delta\left(g_{n}^{\mu \nu}(x, 0)-g_{B n}^{\mu \nu}\right) \\
& \times \delta\left(\partial_{z} g_{n}^{\mu \nu}(x, z)-g_{n}^{\mu \nu^{\prime}}(x, z)\left(\partial_{\nu^{\prime}} \partial_{\mu^{\prime}} G_{x x^{\prime}}\left[g_{\mu \nu, n}(x, z), \varphi_{n}(x, z)\right]\right)_{x^{\prime} \rightarrow x} g_{n}^{\mu^{\prime} \nu}(x, z)\right) \\
& \times \exp \left[-\int d^{D} x \sqrt{g_{n}\left(x, z_{f}\right)}\left\{g_{n}^{\mu \nu}\left(x, z_{f}\right)\left[\partial_{\mu} \phi_{\alpha, n}(x)\right]\left[\partial_{\nu} \phi_{\alpha, n}(x)\right]\right.\right. \\
& \left.+\left[m^{2}-i \varphi_{n}\left(x, z_{f}\right)\right] \phi_{\alpha, n}^{2}(x)\right\} \\
& -N \int d^{D} x \sqrt{g_{n}(x, 0)}\left\{\frac{1}{2 u}\left(\varphi_{n}(x, 0)-i \xi R_{n}(x, 0)\right)^{2}\right\} \\
& -N \int_{0}^{z_{f}} d z \int d^{D} x \sqrt{g_{n}(x, z)}\{ \\
& +\frac{1}{2 u}\left[\partial_{z} \varphi_{n}(x, z)\right]^{2}+\frac{\mathcal{C}_{\varphi}}{2} g_{n}^{\mu \nu}(x, z)\left[\partial_{\mu} \varphi_{n}(x, z)\right]\left[\partial_{\nu} \varphi_{n}(x, z)\right]+\mathcal{C}_{\xi} R_{n}(x, z)\left[\varphi_{n}(x, z)\right]^{2} \\
& \left.\left.+\frac{1}{2 \kappa}\left(R_{n}(x, z)-2 \Lambda\right)\right\}\right] .
\end{aligned}
$$

Following this prescription, we find that the entanglement entropy is composed of two pieces, given by

$$
\mathcal{S}_{E E}\left(z_{f}\right)=\mathcal{S}_{E E}^{\phi_{\alpha}}\left(z_{f}\right)+\mathcal{S}_{E E}^{G R}\left(z_{f}\right)
$$

where $\mathcal{S}_{E E}^{\phi_{\alpha}}\left(z_{f}\right)$ is the entanglement entropy from the matter sector at the IR cutoff $z=z_{f}$ and $\mathcal{S}_{E E}^{G R}\left(z_{f}\right)$ is that from the emergent geometry also at the IR cutoff $z=z_{f}$.

It is straightforward to see that the matter contribution is [58]

$$
\mathcal{S}_{E E}^{\phi_{\alpha}}\left(z_{f}\right)=\left.\int d^{D-2} x_{\perp} \int_{0}^{2 \pi} d \theta \int_{0}^{\infty} d r \sqrt{g_{n}\left(r, x_{\perp}, z_{f}\right)}\left\langle T_{\mu \nu, n}^{\phi_{\alpha}}\left(r, x_{\perp}, z_{f}\right)\right\rangle \frac{\partial g_{n}^{\mu \nu}\left(r, x_{\perp}, z_{f}\right)}{\partial n}\right|_{n=1} .
$$

Here, the energy-momentum tensor is given by

$$
\begin{aligned}
T_{\mu \nu, n}^{\phi_{\alpha}}\left(x, z_{f}\right) \equiv & \frac{1}{\sqrt{g_{n}\left(x, z_{f}\right)}} \frac{\partial \sqrt{g_{n}\left(x, z_{f}\right)} \mathcal{L}_{\phi_{\alpha}}\left[g_{\mu \nu, n}\left(x, z_{f}\right), \varphi_{n}\left(x, z_{f}\right)\right]}{\partial g_{n}^{\mu \nu}\left(x, z_{f}\right)} \\
= & 2\left(\partial_{\mu} \phi_{\alpha, n}(x)\right)\left(\partial_{\nu} \phi_{\alpha, n}(x)\right) \\
& +g_{\mu \nu, n}\left(x, z_{f}\right)\left(g_{n}^{\mu^{\prime} \nu^{\prime}}\left(x, z_{f}\right)\left(\partial_{\mu^{\prime}} \phi_{\alpha, n}(x)\right)\left(\partial_{\nu^{\prime}} \phi_{\alpha, n}(x)\right)\right. \\
& \left.+\left[m^{2}-i \varphi_{n}\left(x, z_{f}\right)\right] \phi_{\alpha, n}^{2}(x)\right)
\end{aligned}
$$

where the effective Lagrangian for the matter sector is $\mathcal{L}_{\phi_{\alpha}}\left[g_{\mu \nu, n}\left(x, z_{f}\right), \varphi_{n}\left(x, z_{f}\right)\right]=$ $g_{n}^{\mu \nu}\left(x, z_{f}\right)\left[\partial_{\mu} \phi_{\alpha, n}(x)\right]\left[\partial_{\nu} \phi_{\alpha, n}(x)\right]+\left[m^{2}-i \varphi_{n}\left(x, z_{f}\right)\right] \phi_{\alpha, n}^{2}(x)$. Inserting this energy- 
momentum tensor into the above expression, we obtain

$$
\begin{aligned}
& \mathcal{S}_{E E}^{\phi_{\alpha}}\left(z_{f}\right)= \\
& \quad N \int d^{D-2} x_{\perp} \int_{0}^{2 \pi} d \theta \int_{0}^{\infty} d r \sqrt{g_{n}\left(r, x_{\perp}, z_{f}\right)}\left\{2\left(\partial_{\mu} \partial_{\nu} G\left[x, x ; g_{\mu \nu, n}\left(x, z_{f}\right), \varphi_{n}\left(x, z_{f}\right)\right]\right)\right. \\
& \quad+g_{\mu \nu, n}\left(r, x_{\perp}, z_{f}\right)\left(g_{n}^{\mu^{\prime} \nu^{\prime}}\left(r, x_{\perp}, z_{f}\right)\left(\partial_{\mu^{\prime}} \partial_{\nu^{\prime}} G\left[x, x ; g_{\mu \nu, n}\left(x, z_{f}\right), \varphi_{n}\left(x, z_{f}\right)\right]\right)\right. \\
& \left.\left.\quad+\left[m^{2}-i \varphi_{n}\left(x, z_{f}\right)\right] G\left[x, x ; g_{\mu \nu, n}\left(x, z_{f}\right), \varphi_{n}\left(x, z_{f}\right)\right]\right)\right\}\left.\frac{\partial g_{n}^{\mu \nu}\left(r, x_{\perp}, z_{f}\right)}{\partial n}\right|_{n=1}
\end{aligned}
$$

where $G\left[x, x^{\prime} ; g_{\mu \nu, n}\left(x, z_{f}\right), \varphi_{n}\left(x, z_{f}\right)\right]=\left\langle\frac{1}{N} \sum_{\alpha=1}^{N} \phi_{\alpha, n}(x) \phi_{\alpha, n}\left(x^{\prime}\right)\right\rangle$ is the Green's function of the original scalar fields at $z=z_{f}$ with the conical singularity. Both the Klein-Gordon action and the induced Einstein-Hilbert gravity action at $z=z_{f}$ in the last two lines result from the gradient expansion of the last logarithmic term [43, 44] in eq. (5.5).

The entanglement entropy from the classical geometry in the presence of dual scalar fields is given by

$$
\begin{aligned}
& \mathcal{S}_{E E}^{G R}\left(z_{f}\right)= \\
& \left.\quad N \int d^{D-2} x_{\perp} \int_{0}^{2 \pi} d \theta \int_{0}^{\infty} d r \int_{0}^{z_{f}} d z \sqrt{g_{n}\left(r, x_{\perp}, z\right)} T_{\mu \nu, n}^{G R}\left(r, x_{\perp}, z\right) \frac{\partial g_{n}^{\mu \nu}\left(r, x_{\perp}, z\right)}{\partial n}\right|_{n=1} .
\end{aligned}
$$

The energy-momentum tensor of the gravity sector is

$$
T_{\mu \nu, n}^{G R}(x, z) \equiv \frac{1}{\sqrt{g_{n}(x, z)}} \frac{\partial \sqrt{g_{n}(x, z)} \mathcal{L}_{G R}\left[g_{\mu \nu, n}(x, z), \varphi_{n}(x, z)\right]}{\partial g_{n}^{\mu \nu}(x, z)} \equiv T_{\mu \nu, n}^{g_{\mu \nu}}(x, z)+T_{\mu \nu, n}^{\varphi}(x, z),
$$

where the bulk Lagrangian in terms of bulk metric tensor and dual scalar fields is given by

$$
\begin{aligned}
\mathcal{L}_{G R}\left[g_{\mu \nu, n}(x, z), \varphi_{n}(x, z)\right]= & \frac{1}{2 u}\left[\partial_{z} \varphi_{n}(x, z)\right]^{2}+\frac{\mathcal{C}_{\varphi}}{2} g_{n}^{\mu \nu}(x, z)\left[\partial_{\mu} \varphi_{n}(x, z)\right]\left[\partial_{\nu} \varphi_{n}(x, z)\right] \\
& +\mathcal{C}_{\xi} R_{n}(x, z)\left[\varphi_{n}(x, z)\right]^{2}+\frac{1}{2 \kappa}\left(R_{n}(x, z)-2 \Lambda\right)
\end{aligned}
$$

As a result, the energy-momentum tensor of our classical gravity is ([59], chapter 2)

$$
\begin{aligned}
2 \kappa T_{\mu \nu, n}^{g_{\mu \nu}}(x, z)= & 2\left(R_{\mu \nu, n}(x, z)-\frac{1}{2} g_{\mu \nu, n}(x, z) R_{n}(x, z)+\Lambda g_{\mu \nu, n}(x, z)\right) \\
& +\frac{1}{g_{n}(x, z)} g_{\mu \mu^{\prime}, n}(x, z) g_{\nu \nu^{\prime}, n}(x, z) \partial_{\mu^{\prime \prime}} \partial_{\nu^{\prime \prime}} \\
& \times\left\{g_{n}(x, z)\left(g_{n}^{\mu^{\prime} \nu^{\prime}}(x, z) g_{n}^{\mu^{\prime \prime} \nu^{\prime \prime}}(x, z)-g_{n}^{\mu^{\prime} \mu^{\prime \prime}}(x, z) g_{n}^{\nu^{\prime} \nu^{\prime \prime}}(x, z)\right)\right\},
\end{aligned}
$$

and that of the dual scalar field is

$$
\begin{aligned}
T_{\mu \nu, n}^{\varphi}(x, z)= & \frac{2}{u} \delta_{\mu z} \delta_{\nu z}\left[\partial_{z} \varphi_{n}(x, z)\right]^{2}+2\left(1-\delta_{\mu z} \delta_{\nu z}\right) \mathcal{C}_{\varphi}\left[\partial_{\mu} \varphi_{n}(x, z)\right]\left[\partial_{\nu} \varphi_{n}(x, z)\right] \\
& +g_{\mu \nu, n}(x, z)\left(\frac{1}{u}\left[\partial_{z} \varphi_{n}(x, z)\right]^{2}+\mathcal{C}_{\varphi} g_{n}^{\mu^{\prime} \nu^{\prime}}(x, z)\left[\partial_{\mu^{\prime}} \varphi_{n}(x, z)\right]\left[\partial_{\nu^{\prime}} \varphi_{n}(x, z)\right]\right) \\
& +2 \mathcal{C}_{\xi}\left(R_{\mu \nu, n}-\frac{1}{2} g_{\mu \nu, n} R_{n}\right)\left[\varphi_{n}(x, z)\right]^{2}+2 \mathcal{C}_{\xi}\left(g_{\mu \nu, n} \partial_{\rho} \partial^{\rho}-\partial_{\mu} \partial_{\nu}\right)\left[\varphi_{n}(x, z)\right]^{2}
\end{aligned}
$$




\subsection{Heat kernel method for entanglement entropy}

To show the entanglement transfer from matter to geometry through recursive renormalization group transformations, we need to simplify the entanglement-entropy formula of the matter sector. Here, we review the entanglement-entropy formula based on the heat kernel method [47]. An effective free energy on $n$-sheeted Riemann with a conical singularity can be represented by

$$
-\ln Z_{n}^{\phi_{\alpha}}\left(z_{f}\right)=-\frac{N}{2} \int_{\epsilon^{2}}^{\infty} \frac{d s}{s} \operatorname{tr}_{x x^{\prime}} K\left(s, x, x^{\prime} ; z_{f}\right)
$$

where $K\left(s, x, x^{\prime} ; z_{f}\right) \equiv\left\langle x z_{f}\left|e^{-s \mathcal{D}}\right| x^{\prime} z_{f}\right\rangle$ is the corresponding heat kernel with the differential operator $\mathcal{D} \equiv-\frac{1}{\sqrt{g\left(x, z_{f}\right)}} \partial_{\mu}\left(\sqrt{g\left(x, z_{f}\right)} g^{\mu \nu}\left(x, z_{f}\right) \partial_{\nu}\right)+m^{2}$. For the time being, we consider the case of a constant mass, given by $m^{2}-i \varphi\left(x, z_{f}\right) \Longrightarrow m^{2}$. This heat kernel satisfies an effective heat-diffusion equation $\left(\partial_{s}+\mathcal{D}\right) K\left(s, x, x^{\prime} ; z_{f}\right)=0$ with an initial condition $K\left(s=0, x, x^{\prime} ; z_{f}\right)=\frac{1}{\sqrt{g\left(x, z_{f}\right)}} \delta^{(D)}\left(x-x^{\prime}\right)$ - this is the origin of the name of the heat kernel. $\epsilon$ is a UV cutoff to cure the UV divergence of the entanglement entropy, which will be clarified below.

To deal with curvature integrals in the presence of a conical singularity, we need to understand the curvature structure of this geometry [47]. Let $E_{\alpha}$ be an $\alpha$-fold Riemann sheet, which covers a smooth manifold $E$ along the Killing vector $\partial_{\theta}$. A codimension two surface $\Sigma$ is a stationary point $(r=0)$ of this isometry. Then, the space $E_{\alpha}$ is a direct product near $\Sigma(r=0)$, given by $\Sigma \times C_{\alpha}$, where $C_{\alpha}$ is a two-dimensional cone with angle deficit $\delta=2 \pi(1-\alpha)$. In other words, the metric of $E_{\alpha}$ is $d s_{E_{\alpha}}^{2}=d s_{C_{\alpha}}^{2}+d s_{\Sigma}^{2}$, where the metric of the two dimensional cone is $d s_{C_{\alpha}}^{2}=g_{r r}^{\alpha}\left(r, x_{\perp}, z\right) d r^{2}+g_{\theta \theta}^{\alpha}\left(r, x_{\perp}, z\right) d \theta^{2}$ and the metric of the codimension two surface is $d s_{\Sigma}^{2}=\gamma_{i j}\left(r, x_{\perp}, z\right) d x_{\perp}^{i} d x_{\perp}^{j}$ at a fixed renormalization group scale $z$. Outside this singular surface $\Sigma, E_{\alpha}$ is reduced to the smooth manifold $E$, where their curvature tensors coincide. On the other hand, the conical singularity at the surface $\Sigma$ gives rise to a singular contribution to the curvatures. Here, the positive integer $n$ is analytically continued to a real value $\alpha$, which results from the abelian isometry generated by the Killing vector $\partial_{\theta}$.

One may take an expansion for small $s$ in the heat kernel, given by

$$
\operatorname{tr}_{x x^{\prime}} K_{E_{\alpha}}(s)=\frac{1}{(4 \pi s)^{\frac{d}{2}}} \sum_{n=0} a_{n} s^{n}
$$

Then, we have two types of coefficients in this expansion,

$$
a_{n}=a_{n}^{r e g}+a_{n}^{\Sigma},
$$

where $a_{n}^{r e g}$ is a regular contribution resulting from the bulk and $a_{n}^{\Sigma}$ is a singular one coming 
from the surface. Regular bulk coefficients are given by

$$
\begin{aligned}
a_{0}^{\mathrm{reg}}= & \int_{E} d^{D} x \sqrt{g\left(x, z_{f}\right)}, \\
a_{1}^{\mathrm{reg}}= & \int_{E} d^{D} x \sqrt{g\left(x, z_{f}\right)}\left(\frac{1}{6} R\left(x, z_{f}\right)-m^{2}\right), \\
a_{2}^{\mathrm{reg}}= & \int_{E} d^{D} x \sqrt{g\left(x, z_{f}\right)}\left\{\frac{1}{180} R_{\mu \nu \alpha \beta}^{2}\left(x, z_{f}\right)-\frac{1}{180} R_{\mu \nu}^{2}\left(x, z_{f}\right)\right. \\
& \left.+\frac{1}{6} \frac{1}{\sqrt{g\left(x, z_{f}\right)}} \partial_{\mu}\left(\sqrt{g\left(x, z_{f}\right)} g^{\mu \nu}\left(x, z_{f}\right) \partial_{\nu}\right)\left(\frac{1}{5} R\left(x, z_{f}\right)-m^{2}\right)+\frac{1}{2}\left(\frac{1}{6} R\left(x, z_{f}\right)-m^{2}\right)^{2}\right\},
\end{aligned}
$$

for general curvature tensors [47]. On the other hand, singular surface coefficients are given by $[47]$

$$
\begin{aligned}
a_{0}^{\Sigma}= & 0 \\
a_{1}^{\Sigma}= & \frac{\pi}{3} \frac{(1-\alpha)(1+\alpha)}{\alpha} \int_{\Sigma\left(z_{f}\right)} d^{D-2} x_{\perp} \sqrt{\gamma\left(x, z_{f}\right)}, \\
a_{2}^{\Sigma}= & \frac{\pi}{3} \frac{(1-\alpha)(1+\alpha)}{\alpha} \int_{\Sigma\left(z_{f}\right)} d^{D-2} x_{\perp} \sqrt{\gamma\left(x, z_{f}\right)}\left(\frac{1}{6} R\left(x, z_{f}\right)-m^{2}\right) \\
& -\frac{\pi}{180} \frac{(1-\alpha)(1+\alpha)\left(1+\alpha^{2}\right)}{\alpha^{3}} \int_{\Sigma\left(z_{f}\right)} d^{D-2} x_{\perp} \sqrt{\gamma\left(x, z_{f}\right)}\left(R_{i i}\left(x, z_{f}\right)-2 R_{i j i j}\left(x, z_{f}\right)\right) .
\end{aligned}
$$

We recall $d s_{\Sigma}^{2}=\gamma_{i j}\left(r, x_{\perp}, z\right) d x_{\perp}^{i} d x_{\perp}^{j}$.

Based on this general curvature formula from the heat kernel expansion, we obtain the following expression of the entanglement entropy for the matter sector

$$
\begin{aligned}
\mathcal{S}_{E E}^{\phi_{\alpha}}\left(z_{f}\right)= & \left.\left(\alpha \partial_{\alpha}-1\right)\left[-\ln Z_{\phi_{\alpha}}\left(\alpha ; z_{f}\right)\right]\right|_{\alpha=1} \\
\approx & -N \frac{\pi}{6} \frac{1}{(4 \pi)^{\frac{D}{2}}}\left\{\left(\alpha \partial_{\alpha}-1\right) \frac{(1-\alpha)(1+\alpha)}{\alpha}\right\}_{\alpha=1} \\
& \times\left(\int_{\epsilon^{2}}^{\infty} \frac{d s}{s} s^{1-\frac{D}{2}}\right)\left\{\int_{\Sigma\left(z_{f}\right)} d^{D-2} x_{\perp} \sqrt{\gamma\left(x, z_{f}\right)}\right\} .
\end{aligned}
$$

This leading contribution results from the $a_{1}^{\Sigma}$ term in eq. (5.17). Considering that the area of the singular surface $\Sigma\left(z_{f}\right)$ is given by

$$
\mathcal{A}\left[\Sigma\left(z_{f}\right)\right]=\int_{\Sigma\left(z_{f}\right)} d^{D-2} x_{\perp} \sqrt{\gamma\left(x, z_{f}\right)}
$$

at the renormalization group scale $z=z_{f}$, we obtain the area law for the entanglement entropy of the matter sector

$$
\mathcal{S}_{E E}^{\phi_{\alpha}}\left(z_{f}\right) \approx \frac{N}{6(D-2)(4 \pi)^{\frac{D}{2}-1}} \frac{\mathcal{A}\left[\Sigma\left(z_{f}\right)\right]}{\epsilon^{D-2}} .
$$


It is straightforward to see that this formula is reduced to the well known result in two spacetime dimensions, given by

$$
\mathcal{S}_{E E}^{\phi_{\alpha}}\left(z_{f}\right)=\frac{N}{6} \ln \frac{\xi}{\epsilon}
$$

Here, $\xi$ is the correlation length proportional to the inverse of the mass parameter $m$, which appears from the introduction of an IR cutoff in the $s$-integral, given by $\int_{\epsilon^{2}}^{\xi^{2}} \frac{d s}{s}$.

Now, we introduce inhomogeneity of the mass term into the above heat-kernel expansion. Then, spacetime derivatives for $\varphi\left(x, z_{f}\right)$ will appear in both expansion coefficients. As far as a disordered phase is concerned, we speculate that this area law would be preserved. It is an interesting question how $\partial_{\mu} \varphi\left(x, z_{f}\right)$ modify the entanglement entropy of the matter sector at quantum criticality. In this paper, we continue our discussions for the gapped phase.

Finally, we obtain the total entanglement entropy at $z=z_{f}$

$$
\begin{aligned}
\mathcal{S}_{E E}\left(z_{f}\right)= & \mathcal{S}_{E E}^{\phi_{\alpha}}\left(z_{f}\right)+\mathcal{S}_{E E}^{G R}\left(z_{f}\right) \\
= & \frac{N}{6(D-2)(4 \pi)^{\frac{D}{2}-1}} \frac{\mathcal{A}\left[\Sigma\left(z_{f}\right)\right]}{\epsilon^{D-2}} \\
& +N \int d^{D-2} x_{\perp} \int_{0}^{2 \pi} d \theta \int_{0}^{\infty} d r \int_{0}^{z_{f}} d z \sqrt{g_{n}\left(r, x_{\perp}, z\right)} \\
& \times\left.\left(T_{\mu \nu, n}^{\varphi}\left(r, x_{\perp}, z\right)+T_{\mu \nu, n}^{g_{\mu \nu}}\left(r, x_{\perp}, z\right)\right) \frac{\partial g_{n}^{\mu \nu}\left(r, x_{\perp}, z\right)}{\partial n}\right|_{n=1} .
\end{aligned}
$$

In this formula we focus on the fact that the entanglement entropy of the matter sector is given by the area of the subsystem at a fixed renormalization group scale $z=z_{f}$ while that of the classical gravity part is given by the integral of the extra dimensional space. If the emergent geometry is given by a cap in the extra dimensional space, the area of the subsystem at a given $z=z_{f}$ vanishes beyond the cap in the extra dimensional space. Instead, the entanglement entropy from the classical geometry is maximized. Actually, the cap geometry has been realized when the corresponding quantum state is gapped [33, 48, 49]. Even if the resulting geometry is given by $\mathrm{AdS}_{D+1}$, the entanglement entropy is transferred from quantum matter to classical gravity.

\subsection{Invariance of entanglement entropy with respect to renormalization group transformations}

Since the partition function (5.3) has to be invariant with respect to recursive renormalization group transformations, we obtain the following equation

$$
\partial_{z_{f}} \mathcal{S}_{E E}\left(z_{f}\right)=\partial_{z_{f}} \mathcal{S}_{E E}^{\phi_{\alpha}}\left(z_{f}\right)+\partial_{z_{f}} \mathcal{S}_{E E}^{g_{\mu \nu}}\left(z_{f}\right)=0 \Longrightarrow \mathcal{S}_{E E}\left(z_{f}\right)=\mathcal{S}_{E E}^{\phi_{\alpha}}(0)
$$

In other words, the entanglement entropy is an invariant for renormalization group transformations. Resorting to the full expression eq. (5.22) of the entanglement entropy, we 
reach the following identity

$$
\begin{aligned}
& \frac{N}{6(D-2)(4 \pi)^{\frac{D}{2}-1}} \frac{\partial_{z_{f}} \mathcal{A}\left[\Sigma\left(z_{f}\right)\right]}{\epsilon^{D-2}} \\
& +N \int d^{D-2} x_{\perp} \int_{0}^{2 \pi} d \theta \int_{0}^{\infty} d r \sqrt{g_{n}\left(r, x_{\perp}, z_{f}\right)} \\
& \times\left.\left(T_{\mu \nu, n}^{\varphi}\left(r, x_{\perp}, z_{f}\right)+T_{\mu \nu, n}^{g_{\mu \nu}}\left(r, x_{\perp}, z_{f}\right)\right) \frac{\partial g_{n}^{\mu \nu}\left(r, x_{\perp}, z_{f}\right)}{\partial n}\right|_{n=1} \\
& =0 .
\end{aligned}
$$

We suspect that the bulk gravity contribution may be related with the holographic entanglement entropy of the Ryu-Takayanagi formula [36,37]. In particular, one may express this identity in terms of fully geometric information such as metric and curvature. In appendix B, we discuss this identity further and verify the first iteration version of this identity.

\section{Discussion}

\subsection{Remarks on the appearance of higher-spin fields}

One may suggest an effective higher spin gauge theory as a dual holographic description of the $O(N)$ vector model, which has an infinite tower of higher-spin fields without hierarchy in the spectrum [51-54]. Such higher-spin fields can result from the procedure to reformulate the non-local quadratic term generated by the renormalization group transformation in a local way, where emergent bi-local fields such as

$$
-\int d^{D} x \sqrt{g_{x}^{(0)}} \int d^{D} x^{\prime} \sqrt{g_{x^{\prime}}^{(0)}} g_{x}^{\mu \nu(0)} g_{x^{\prime}}^{\mu^{\prime} \nu^{\prime}(0)}\left(\partial_{\mu} \phi_{\alpha x}\right)\left(\partial_{\nu} \partial_{\mu^{\prime}} G_{x x^{\prime}}^{(0)}\right)\left(\partial_{\nu^{\prime}} \phi_{\alpha x^{\prime}}\right)
$$

in eq. (3.27) are rewritten in terms of higher-spin fields. It has been discussed that the origin of higher-spin fields is the nonlocal diffeomorphism invariance [55-57]. Here, we show that the nonlocal diffeomorphism invariance is explicitly broken by the existence of effective interactions. Then, we argue that our effective dual holographic mean-field theory in the large $N$ limit gives a physically meaningful description for strongly coupled field theories even if we do not take into account higher-spin fields.

First, to discuss the absence of the non-local reparameterization symmetry with physically simple notations, we consider a discrete version of the present effective field theory, given by

$$
\mathcal{S}_{\mathrm{eff}}=-\sum_{i j} t_{i j} \phi_{i} \phi_{j}+m^{2} \sum_{i} \phi_{i}^{2}+\frac{u}{2} \sum_{i}\left(\phi_{i}^{2}\right)^{2}
$$

where we put the continuum field theory on a D-dimensional lattice and the indices $i, j$ label lattice sites. The quadratic part enjoys the invariance under the non-local reparameterization $[33,55-57]$

$$
\phi_{i} \longrightarrow \sum_{j} V_{i j} \phi_{j}, \quad t_{i j} \longrightarrow \sum_{i^{\prime} j^{\prime}} V_{i i^{\prime}} t_{i^{\prime} j^{\prime}} V_{j j^{\prime}}
$$


where $V_{i j}$ is an orthogonal matrix acting on the space of all sites. This nonlocal diffeomorphism invariance at the quadratic level, however, is explicitly broken by the last selfinteraction term: in this paper, we considered the Hubbard-Stratonovich transformation as follows

$$
\frac{u}{2} \sum_{i}\left(\phi_{i}^{2}\right)^{2} \longrightarrow \frac{1}{2 u} \sum_{i} \varphi_{i}^{2}-i \sum_{i} \varphi_{i} \phi_{i}^{2}
$$

where $\varphi_{i}$ is a collective dual order-parameter field. Applying the nonlocal coordinate transformation to $\sum_{i} \varphi_{i} \phi_{i}^{2}$, we obtain

$$
\sum_{i} \varphi_{i} \phi_{i}^{2} \longrightarrow \sum_{k} \sum_{j} \phi_{k}\left(\sum_{i} \varphi_{i} V_{k i} V_{i j}\right) \phi_{j}
$$

It is clear that if the bulk solution of $\varphi_{i}$ does not satisfy $\sum_{i} V_{k i} \varphi_{i} V_{i j} \neq \varphi_{j} \delta_{k j}$, this nonlocal diffeomorphism invariance is broken down explicitly.

However, we would like to point out that the vacuum solution of the dual orderparameter field is possibly translational invariant, given by a constant value in the Ddimensional Euclidean space. Then, we suspect the existence of such higher-spin fields as a vacuum solution. On the other hand, excitations of higher-spin fields break the nonlocal diffeomorphism invariance, which would generate gaps for these higher-spin fields. Still, they may not be ignored in principle because the gap would be at most $O(1)$ in the unit of the curvature of the bulk. Frankly speaking, we do not understand what happens in this situation. In order to see higher-spin fields appearing in interacting theories, for example, we may have to properly modify the non-local diffeomorphisms.

Next, we discuss physics of our effective dual holographic mean-field theory in the large $N$ limit. We claim that this dual holographic effective field theory still deserves to be investigated, considering that (i) the partition function derived from this recursive renormalization group transformation is the same as that resulting from a recursive Kadanoff block-spin transformation, verified in one spatial dimension, (ii) the resulting classical field theory in the large $N$ limit takes into account quantum corrections in the all-loop order, thus serving as a novel mean-field theory framework with non-perturbative quantum corrections, and (iii) a comparison of the current holographic approach with the Bethe ansatz solution in the Kondo problem implies that the resulting (dual holographic) effective field theory takes into account quantum corrections in a non-perturbative way.

- Recently, we studied an effective scalar field theory on a one-dimensional lattice, by using the recursive Kadanoff block-spin transformation [60]. As a result, we obtained an effective dual holographic partition function, where renormalization group $\beta$-function of all the coupling functions and the bulk effective action for the dual orderparameter field appears in the background of renormalized coupling functions. The emergent extra dimension corresponds to the iteration number of Kadanoff block-spin transformations.

Here, the conventional truncation scheme of the renormalization group transformation was utilized in the recursive Kadanoff block-spin transformation, where only local 
terms are kept to preserve the original form of the effective Hamiltonian. This appears to be essentially the same approximation we used in our gravitational/geometrical formulation presented in this paper. In fact, by comparing this effective field theory derived from the recursive Kadanoff block-spin transformation with the present geometric formulation (when energy-momentum tensor-type interactions are turned off and thus, the metric tensor is not dynamical, described by the delta-function constraint), we can establish that these two partition functions are equivalent up to a vacuum energy contribution. In particular, we could represent the renormalization group flow of the metric tensor (the present real-space renormalization group scheme) in terms of the running mass parameter (the Kadanoff block-spin renormalization group scheme).

The equivalence between these two formulations indicates that the truncation in the geometrical renormalization group formulation presented in this paper is a physically considerable one or at least as conventional, and often successful, as the truncation in the Kadanoff block spin transformations. With these truncations, higher-spin fields appear neither in the Kadanoff block-spin renormalization group scheme nor in the geometric formulation presented in this paper.

- Furthermore, we also proposed how to extract renormalization coefficients such as field renormalization, mass renormalization, and interaction renormalization constants from the present dual holographic effective field theory in a general ground. Reformulating the renormalized effective field theory with such renormalized coefficients in an effective holographic way, we could determine all these renormalization constants from the present effective geometry in a non-perturbative way [61]. It turns out that this proposal shares essentially the same spirit as the holographic renormalization group formulation [5-7] for the renormalized effective on-shell action.

Finally, let us recapitulate the essential ingredients of this effective mean-field theory in the large $N$ limit. The crucial step in this recursive renormalization group transformation is the renormalization group transformation given by the path integral of the heavy (large mass) scalar (original matter) fields. As a result of this renormalization group transformation, the metric tensor and the dual order-parameter field become truly renormalized. As discussed above, the renormalization group flow of the metric tensor correspond to the renormalization group $\beta$-functions of the coupling functions. An interesting point is that the renormalization group flow of the metric tensor is given by the Green's function of the high-energy scalar fields, which is the only dynamical information in this renormalization group transformation. If one looks into this Green's function more carefully, it depends not only on the dual order parameter field but also on the metric tensor. In other words, self-energy corrections given by the renormalization group flow of the dual order parameter field and vertex corrections given by that of the metric tensor are self-consistently and non-linearly intertwined, which takes into account renormalization effects non-perturbatively. Indeed, the IR boundary condition of the dual order parameter field corresponds to a mean-field 
equation of the order parameter field but with full renormalization effects given by the self-consistent renormalization group flow of the coupling functions [60,61].

- In ref. [62], we applied momentum-space renormalization group transformations to the Kondo problem. Here, we separate slow and fast modes for each field in frequency space, and perform renormalization group transformations in a recursive way. As a result, we were able to describe the crossover regime from the high-temperature decoupled local-moment fluctuating regime to the low-temperature Kondo-singlet localFermi-liquid state, where log-divergences are fully resummed through this recursive renormalization group framework. Unfortunately, we could not introduce the gravitational field to this momentum-space (or frequency-space) renormalization group transformation scheme, where the resulting effective action should be non-local in the reciprocal space, which prohibits us from applying the conventional renormalization group transformation scheme. Nevertheless, it turned out that both the specific heat and the spin susceptibility for the impurity dynamics show a reasonable match to those of the Bethe ansatz solution. This remarkable result implies that the resulting (dual holographic) effective field theory takes into account quantum corrections in a non-perturbative way, i.e., in the all-loop order, although this does not mean that this solution is exact.

\subsection{Remarks on the linear approximation for the dynamics of the metric ten- sor in the recursive renormalization group transformation}

We point out that our linear approximation in the recursive renormalization group transformation for the gravity sector gives rise to a linearized Einstein-scalar theory along the $z$-directional emergent space. However, we argue below that the effective bulk gravity theory contains more than that of a linearized Einstein-scalar theory. In particular, we claim that this effective action allows a higher derivative curvature term. To overcome the limitation of this linear approximation, we suspect that the full diffeomorphism invariance including the extra-dimensional space has to be introduced into the bulk effective action. Although we do not have any concrete formulation yet, we discuss how the full nonlinearity of gravity can be taken into account consistently with the IR boundary condition for matching between microscopic and macroscopic degrees of freedom.

Previously, we discussed that the renormalization group flow of the metric tensor gives rise to all-loop order renormalization effects of the coupling functions, non-linearly intertwined with the renormalization group flow of the dual order-parameter field, even if the metric tensor is not dynamical. We recall the renormalization group flow of the metric tensor, given by $\partial_{z} g^{\mu \nu}(x, z)=\beta_{g}^{\mu \nu}\left[g^{\mu \nu}(x, z), \varphi(x, z)\right]$, where the $\beta$-function is $\beta_{g}^{\mu \nu}\left[g^{\mu \nu}(x, z), \varphi(x, z)\right]=g^{\mu \nu^{\prime}}(x, z)\left(\partial_{\nu^{\prime}} \partial_{\mu^{\prime}} G_{x x^{\prime}}\left[g^{\mu \nu}(x, z), \varphi(x, z)\right]\right)_{x^{\prime} \rightarrow x} g^{\mu^{\prime} \nu}(x, z)$.

First, to see how higher derivative terms can arise, we consider the case when the metric tensor is dynamical, where the effective action of the metric tensor is given by

$$
\begin{aligned}
\mathcal{S}_{\text {grav. }}= & N \int_{0}^{z_{f}} d z \int d^{D} x \sqrt{g(x, z)}\left\{-\frac{1}{2 \lambda}\left(\partial_{z} g^{\mu \nu}(x, z)-\beta_{g}^{\mu \nu}\left[g^{\mu \nu}(x, z), \varphi(x, z)\right]\right)^{2}\right. \\
& \left.+\frac{1}{2 \kappa}(R(x, z)-2 \Lambda)\right\} .
\end{aligned}
$$


To develop an intuitive picture on the role of the first term, it is tempting to regard $\beta_{g}^{\mu \nu}\left[g^{\mu \nu}(x, z), \varphi(x, z)\right]$ as the Ricci tensor, $R_{\mu \nu}(x, z)$; we consider

$$
\begin{aligned}
\mathcal{S}_{\text {grav. }}= & N \int_{0}^{z_{f}} d z \int d^{D} x \sqrt{g(x, z)}\left\{-\frac{1}{2 \lambda}\left(\partial_{z} g^{\mu \nu}(x, z)+2 R^{\mu \nu}(x, z)\right)\left(\partial_{z} g_{\mu \nu}(x, z)+2 R_{\mu \nu}(x, z)\right)\right. \\
& \left.+\frac{1}{2 \kappa}(R(x, z)-2 \Lambda)\right\} .
\end{aligned}
$$

This is natural in the sense that the resulting evolution equation for the metric tensor in the $\lambda \rightarrow 0$ limit is given by the Ricci flow equation [63-68]

$$
\partial_{z} g_{\mu \nu}(x, z)=-2 R_{\mu \nu}(x, z) .
$$

The Ricci flow equation is to describe the deformation of a Riemannian metric $g_{\mu \nu}(x, z)$ with an extra-dimensional space coordinate $z$, here, which plays the same role as time. $\mu$ and $\nu$ cover the $D$-dimensional spacetime coordinate $0, \ldots, D-1$. This evolution equation may be regarded as an analog of the diffusion equation for geometries, given by a parabolic partial differential equation. The deformation is governed by Ricci curvature, which leads to homogeneity of geometry. In principle, one may consider that this Ricci flow equation arises from the gradient expansion of the Green's function with respect to the mass parameter, where additional terms given by curvature gradients and dual order-parameter fields are all neglected. See the Green's function formula of eq. (B.4) in appendix B.1.

Here, we would also like to point out that the holographic renormalization group flow gives rise to the Ricci flow naturally [69, 70]. Actually, our holographic dual effective field theory turns out to reproduce this result at the fixed point of the $z_{f} \rightarrow \infty$ limit.

To verify the above statement, we consider the Hamilton-Jacobi formulation for the holographic dual effective field theory, given by

$$
\begin{aligned}
& \frac{1}{\sqrt{g\left(x, z_{f}\right)}} \frac{\partial}{\partial z_{f}} \mathcal{I}\left[\varphi\left(x, z_{f}\right), g_{\mu \nu}\left(x, z_{f}\right)\right]=\frac{N \lambda}{2}\left\{\frac{1}{\sqrt{g\left(x, z_{f}\right)}} \frac{\partial \mathcal{I}\left[\varphi\left(x, z_{f}\right), g_{\mu \nu}\left(x, z_{f}\right)\right]}{\partial g^{\mu \nu}\left(x, z_{f}\right)}\right\}^{2} \\
& +N \beta_{\mu \nu}^{g}\left[\varphi\left(x, z_{f}\right), g_{\mu \nu}\left(x, z_{f}\right)\right]\left\{\frac{1}{\sqrt{g\left(x, z_{f}\right)}} \frac{\partial \mathcal{I}\left[\varphi\left(x, z_{f}\right), g_{\mu \nu}\left(x, z_{f}\right)\right]}{\partial g^{\mu \nu}\left(x, z_{f}\right)}\right\}+\frac{N}{2 \kappa}\left(R\left(x, z_{f}\right)-2 \Lambda\right),
\end{aligned}
$$

where $\mathcal{I}\left[\varphi\left(x, z_{f}\right), g_{\mu \nu}\left(x, z_{f}\right)\right]$ is an IR effective on-shell action. We recall our gauge fixing for the metric tensor, given by $g_{D D}(x, z)=1$ and $g_{\mu D}(x, z)=0$ with $\mu=0, \ldots, D-1$. Here, we focused on the gravitational part only. We point out that the $\beta$-function has to vanish in the $z_{f} \rightarrow \infty$ limit corresponding to a fixed point. When this $\beta$-function of the metric tensor vanishes at the fixed point, this Hamilton-Jacobi equation is essentially reduced into that of the holographic duality conjecture [5-7], which reproduces the Ricci flow $[69,70]$. On the other hand, when the $\beta_{g}^{\mu \nu}\left[\varphi\left(x, z_{f}\right), g_{\mu \nu}\left(x, z_{f}\right)\right]$ function does not vanish, it depends on both $\varphi\left(x, z_{f}\right)$ and $g_{\mu \nu}\left(x, z_{f}\right)$ through the Green's function in an intertwined and nonlinear way, and this nonlinear intertwined renormalization structure gives rise to difficulty in solving this Hamilton-Jacobi equation. The present prescription generalizes 
the holographic renormalization group flow of the AdS geometry towards that without conformal symmetry. More detailed discussions can be found in section 4. Discussion: Ricci Flow of ref. [61].

Resorting to the Ricci flow in the holographic renormalization, we conclude that the linear approximation for the metric tensor in the recursive renormalization group transformation gives rise to a higher-order curvature term such as $R^{\mu \nu}(x, z) R_{\mu \nu}(x, z)$ in the Einstein-Hilbert action. We currently do not know how to justify the linear approximation for the evolution of the metric tensor in the recursive renormalization group procedure, except that we use the same approximation for the heavy dual scalar field. Nevertheless, even within the linear approximation, the appearance of the higher derivative curvature term is an interesting feature, rather unexpected.

Second, we discuss how to introduce full nonlinearity into the above dual holographic effective field theory. Although we do not have any concrete idea for the first-principle derivation in this issue, we suspect that full diffeomorphism invariance has to be satisfied, which requires to extend the present formulation, including both spacetime dependent lapse function and shift vector fields [72]. In this respect we suggest the following dual holographic effective field theory

$$
Z=\int D \phi_{\alpha}(x) D \mathcal{G}^{M N}(x, z) D \varphi(x, z) \exp \left\{-\mathcal{S}_{\mathrm{Bulk}}-\mathcal{S}_{\mathrm{IR}}-\mathcal{S}_{\mathrm{UV}}\right\} .
$$

Here, one of the two key points is that the bulk effective action enjoys the full $(D+$ 1)-dimensional diffeomorphism invariance, given by

$$
\begin{aligned}
\mathcal{S}_{\text {Bulk }}= & N \int_{0}^{z_{f}} d z \int d^{D} x \sqrt{\mathcal{G}(x, z)}\left(\frac{1}{2} \mathcal{G}^{M N}\left(\partial_{M} \varphi(x, z)\right)\left(\partial_{N} \varphi(x, z)\right)+\mathcal{C}_{\xi} \mathcal{R}(x, z)[\varphi(x, z)]^{2}\right. \\
& \left.+\frac{1}{2 \kappa}\{\mathcal{R}(x, z)-2 \Lambda\}+\text { higher-derivative curvature terms }\right),
\end{aligned}
$$

where $M$ and $N$ are $(D+1)$-dimensional coordinates including the emergent extradimensional space. This bulk effective action may be regarded as a conventional holographic bulk action although it is extended to include higher-derivative curvature terms as discussed above. The IR boundary effective action is

$$
\begin{aligned}
\mathcal{S}_{\mathrm{IR}}= & \int d^{D} x \sqrt{g\left(x, z_{f}\right)}\left(g^{\mu \nu}\left(x, z_{f}\right)\left(\partial_{\mu} \phi_{\alpha}(x)\right)\left(\partial_{\nu} \phi_{\alpha}(x)\right)+\left(m^{2}-i \varphi\left(x, z_{f}\right)\right)\left[\phi_{\alpha}(x)\right]^{2}\right. \\
& \left.+\xi R\left(x, z_{f}\right)\left[\phi_{\alpha}(x)\right]^{2}\right)+ \text { GHY boundary term }
\end{aligned}
$$

where $g^{\mu \nu}\left(x, z_{f}\right)$ represent fully renormalized coupling functions as discussed before, which have to be identified with an induced metric tensor from the bulk metric. Obviously, $\mu$ and $\nu$ span the boundary $D$-dimensional spacetime coordinate at $z=z_{f}$. In addition, the Gibbons-Hawking-York boundary term has to be taken into account from the bulk effective action $[45,46]$. Finally, the UV boundary action is given by

$$
\begin{aligned}
\mathcal{S}_{\mathrm{UV}}= & N \int d^{D} x \sqrt{g(x, 0)}\left(\frac{1}{2 u}\left(\varphi(x, 0)-\varphi^{e x t}(x)\right)^{2}+\mathcal{C}_{\xi} R(x, 0)[\varphi(x, 0)]^{2}\right) \\
& + \text { GHY boundary term },
\end{aligned}
$$


essentially the same as the presently derived version of the UV boundary condition, where the UV boundary metric tensor is nothing but Euclidean. Here, we introduced an external source term $\varphi^{\text {ext }}(x)$ to measure density-fluctuation correlations.

Compared to the conventional approach in the dual holographic theory, the only novel aspect is to introduce the IR boundary effective action explicitly into the framework. Thus, the last of the two key points is on how to incorporate the IR boundary condition, which has to be consistent with the bulk effective action. An idea is to consider the ADM Hamiltonian gravity formulation [72] for the bulk effective action. Then, the bulk effective action is rewritten as follows formally

$$
\mathcal{S}_{\mathrm{Bulk}}^{\mathrm{ADM}}=N \int_{0}^{z_{f}} d z \int d^{D} x\left(\pi_{\mu \nu}(x, z) \partial_{z} g^{\mu \nu}(x, z)-N(x, z) H(x, z)-N^{\mu}(x, z) H_{\mu}(x, z)\right),
$$

where $\pi_{\mu \nu}(x, z)$ is the canonical conjugate variable to $g^{\mu \nu}(x, z), N(x, z)$ is the lapse function, and $N^{\mu}(x, z)$ is the shift vector field. As a result, we have two constraint equations, given by $H(x, z)=0$, which can be identified with the Hamilton-Jacobi equation in the holographic renormalization framework, and $H^{\mu}(x, z)=0$, which gives rise to the Ward identity involved with the $D$-dimensional diffeomorphism invariance [73]. Solving the Hamilton-Jacobi equation, we suspect that the resulting on-shell effective action has to be consistent with the IR boundary effective action, where their consistency is guaranteed by the corresponding Ward identity of $H^{\mu}(x, z)=0$. This IR-boundary matching procedure makes the connection between UV microscopic degrees of freedom and IR emergent macroscopic ones be complete. However, we would like to point out that actual implementations have to be performed carefully.

One may concern that the IR boundary effective action may not be uniquely determined. Suppose that the IR boundary action is given by [74]

$$
\begin{aligned}
\mathcal{S}_{\mathrm{IR}}= & \int d^{D} x \sqrt{g\left(x, z_{f}\right)}\left(\bar{\psi}_{\alpha}(x) \gamma^{a} e_{a}^{\mu}\left(x, z_{f}\right)\left(\partial_{\mu}-\frac{i}{4} \omega_{\mu}^{a^{\prime} b^{\prime}}\left(x, z_{f}\right) \sigma_{a^{\prime} b^{\prime}}\right) \psi_{\alpha}(x)\right. \\
& \left.+\left[m-i \varphi\left(x, z_{f}\right)\right] \bar{\psi}_{\alpha}(x) \psi_{\alpha}(x)\right)+ \text { GHY boundary term }
\end{aligned}
$$

where "chiral symmetry breaking" in interacting Dirac-fermion systems [75] has been considered. Here, $\psi_{\alpha}(x)$ is a Dirac spinor at $x$ in $D$ spacetime dimensions. $\alpha$ runs from 1 to $N$, denoting the flavor degeneracy of Dirac fermions. $\gamma^{a}$ is a Dirac $\gamma$ matrix, defined in a local rest frame at $x$ and satisfying the Clifford algebra $\left\{\gamma^{a}, \gamma^{b}\right\}=2 \delta^{a b}$ with the Euclidean signature. $e_{a}^{\mu}\left(x, z_{f}\right)$ defines the local rest frame given by the tangent manifold at $x$, called vierbein. The corresponding background metric is given by the vierbein as follows $g_{\mu \nu}\left(x, z_{f}\right)=$ $e_{\mu}^{a}\left(x, z_{f}\right) e_{\nu}^{b}\left(x, z_{f}\right) \delta_{a b} . \omega_{\mu}^{a b}\left(x, z_{f}\right)=e_{\nu}^{a}\left(x, z_{f}\right) \partial_{\mu} e^{\nu b}\left(x, z_{f}\right)+e_{\nu}^{a}\left(x, z_{f}\right) \Gamma_{\sigma \mu}^{\nu}\left(x, z_{f}\right) e^{\sigma b}\left(x, z_{f}\right)$ is a spin connection and $\sigma_{a b}=\frac{i}{2}\left[\gamma^{a}, \gamma^{b}\right]$ is a commutator of Dirac gamma matrices in the local rest frame. Here, $\Gamma_{\sigma \mu}^{\nu}\left(x, z_{f}\right)=\frac{1}{2} g^{\nu \delta}\left(x, z_{f}\right)\left(\partial_{\sigma} g_{\delta \mu}\left(x, z_{f}\right)+\partial_{\mu} g_{\sigma \delta}\left(x, z_{f}\right)-\partial_{\delta} g_{\sigma \mu}\left(x, z_{f}\right)\right)$ is the Christoffel symbol. $m$ represents a mass of Dirac fermions. $\varphi\left(x, z_{f}\right)$ is an order-parameter field dual to $\frac{1}{N} \sum_{\alpha=1}^{N} \bar{\psi}_{\alpha}(x) \psi_{\alpha}(x)$ at $z=z_{f}$. Resorting to the ADM Hamiltonian gravity formulation, we assign the IR boundary condition to the same bulk effective action but in 
terms of microscopic fermion degrees of freedom instead of bosons. Solving two coupled equations for dual scalar fields and metric tensor fields modified by higher-derivative curvature terms, we would find effective geometry as a function of the mass parameter or more precisely, the ratio between the mass parameter and the interaction strength. Although we resort to the same bulk effective action, we suspect that both aspects of the difference in the IR boundary condition and the presence of higher-derivative curvature terms may lead to different geometries or different conformal dimensions at least, when the mass parameter is tuned to cause a quantum phase transition. This would be certainly a future direction of our research.

\section{Conclusion}

In the present study we tried to clarify the underlying physics of the dual holographic formulation for quantum field theories as a non-perturbative description. We uncovered how the emergent geometry takes into account the information of quantum entanglement. Let us recapitulate the essential features of the dual holographic formulation. First, the infinitesimal distance in the emergent extra dimensional space is identified with the renormalization group scale in the recursive renormalization group transformation. Second, the IR boundary condition describes the renormalization group $\beta$-functions of all the coupling parameters in the corresponding quantum field theory. In particular, the IR boundary conditions given by metric tensor fields are identified with the renormalization group equations of all interaction vertices and that given by the scalar field is nothing but the CallanSymanzik equation for an order parameter field in the dual description. Finally, the bulk Einstein-Klein-Gordon type equation describes the entanglement transfer from quantum matter to classical geometry in the large $N$ limit, where $N$ is the number of flavors of scalar fields.

The future direction in this line of research would be to solve the resulting coupled equations of motion in the emergent curved spacetime with an extra dimension and to show the appearance of hydrodynamic behaviors at IR, for example, calculating the ratio $\eta / s$, where $\eta$ is shear viscosity and $s$ is thermodynamic entropy [76-79]. Recently, one of the authors has investigated the Kondo effect based on this emergent geometric description and was able to describe the crossover behavior around the Kondo temperature from the UV local-moment fixed point to the IR local Fermi-liquid one in a non-perturbative way [62]. The so-called holographic liquid state may serve as a novel quantum liquid beyond the perturbation theoretical framework.

\section{Acknowledgments}

K.-S. Kim was supported by the Ministry of Education, Science, and Technology (No. 2011-0030046 and NRF-2021R1A2C1006453) of the National Research Foundation of Korea (NRF) and by TJ Park Science Fellowship of the POSCO TJ Park Foundation. This work was supported by a grant from the Simons Foundation (566116, SR). 


\section{A Emergent geometric description for a scalar lattice field theory in one dimension}

We start from the following one-dimensional lattice field theory

$$
Z=\int D \Phi_{i} \exp \left[-\int_{0}^{\beta} d \tau \sum_{i=1}^{M}\left\{\left(\partial_{\tau} \Phi_{i}\right)^{2}-t\left(\Phi_{i} \Phi_{i+1}+\Phi_{i+1} \Phi_{i}\right)+m^{2} \Phi_{i}^{2}+\frac{u}{2} \Phi_{i}^{4}\right\}\right]
$$

Here, $\Phi_{i}$ is a real scalar field at site $i . t$ is a hopping integral and $m$ is a mass parameter. $u$ represents the strength of self-interactions between these scalar fields.

To implement recursive Kadanoff block-spin transformations [71] in this lattice field theory, we uplift all coupling parameters into dynamical field variables as follows

$$
\begin{aligned}
Z= & \int D \Phi_{i} D \varphi_{i}^{(0)} D \rho_{i}^{(0)} D s_{i i+1}^{(0)} D t_{i i+1}^{(0)} D \chi_{i}^{(0)} D m_{i}^{2(0)} D v_{i}^{(0)} D u_{i}^{(0)} \\
& \times \exp \left[-\int_{0}^{\beta} d \tau \sum_{i=1}^{M}\left\{\left(\partial_{\tau} \Phi_{i}\right)^{2}-t_{i i+1}^{(0)}\left(\Phi_{i} \Phi_{i+1}+\Phi_{i+1} \Phi_{i}\right)+m_{i}^{2(0)} \Phi_{i}^{2}+\frac{u_{i}^{(0)}}{2} \rho_{i}^{(0) 2}\right.\right. \\
& \left.\left.+i \varphi_{i}^{(0)}\left(\rho_{i}^{(0)}-\Phi_{i}^{2}\right)+i s_{i i+1}^{(0)}\left(t_{i i+1}^{(0)}-t\right)+i \chi_{i}^{(0)}\left(m_{i}^{2(0)}-m^{2}\right)+i v_{i}^{(0)}\left(u_{i}^{(0)}-u\right)\right\}\right] .
\end{aligned}
$$

Here, $s_{i i+1}^{(0)}, \chi_{i}^{(0)}$, and $v_{i}^{(0)}$ are Lagrange multiplier fields to impose each constraint for hopping, mass, and self-interaction parameters, respectively. $\varphi_{i}^{(0)}$ is a Lagrange multiplier field to realize the Hubbard-Stratonovich transformation for the self-interaction channel.

Separating all dynamical fields at each site into those at even and odd sites, integrating over all field variables in even sites, and rescaling to return the lattice to the original one, we obtain an effective lattice field theory with renormalized hopping, mass, self-interaction, and dual scalar fields as follows

$$
\begin{aligned}
Z= & \int D \Phi_{i} D \varphi_{i}^{(0)} D \rho_{i}^{(0)} D s_{i i+1}^{(0)} D t_{i i+1}^{(0)} D \chi_{i}^{(0)} D m_{i}^{2(0)} D v_{i}^{(0)} D u_{i}^{(0)} \\
& \times \exp \left[-\frac{1}{4} \sum_{i=1}^{M} \operatorname{tr}_{\tau \tau^{\prime}} \ln \left(-\partial_{\tau}^{2}+m_{i}^{2(0)}\right)\right. \\
& -\int_{0}^{\beta} d \tau \sum_{i=1}^{M}\left\{\left(\partial_{\tau} \Phi_{i}\right)^{2}+\left(m_{i}^{2(0)}-\frac{2 m_{i}^{2(0)} t_{i i+1}^{(0) 2}}{2 m_{i}^{4(0)}+u_{i}^{(0)}}\right) \Phi_{i}^{2}-i \varphi_{i}^{(0)} \Phi_{i}^{2}\right. \\
& \left.+\frac{u_{i}^{(0)}}{2} \rho_{i}^{(0) 2}+i \varphi_{i}^{(0)} \rho_{i}^{(0)}+i s_{i i+1}^{(0)}\left(t_{i i+1}^{(0)}-t\right)+i \chi_{i}^{(0)}\left(m_{i}^{2(0)}-m^{2}\right)+i v_{i}^{(0)}\left(u_{i}^{(0)}-u\right)\right\} \\
& -\int_{0}^{\beta} d \tau \sum_{i=1}^{M}\left\{-\frac{t_{i i+1}^{(0) 2}}{2 m_{i}^{4(0)}+u_{i}^{(0)}}\left\{m_{i}^{2(0)}-\frac{u_{i}^{(0)} t_{i i+1}^{(0) 2}}{m_{i}^{4(0)}}\left(\Phi_{i}^{2}+\Phi_{i+1}^{2}\right)\right\}\left(\Phi_{i} \Phi_{i+1}+\Phi_{i+1} \Phi_{i}\right)\right. \\
& \left.\left.+\frac{u_{i}^{(0)}}{2 m_{i}^{4(0)}+u_{i}^{(0)}}\left\{\frac{t_{i i+1}^{(0) 4}}{m_{i}^{4(0)}} \Phi_{i}^{4}+3 \frac{t_{i i+1}^{(0) 4}}{m_{i}^{4(0)}} \Phi_{i}^{2} \Phi_{i+1}^{2}\right\}+\frac{1}{16 m_{i}^{4(0)}}\left(\frac{1}{u_{i}^{(0)}}+\frac{1}{2 m_{i}^{4(0)}}\right)^{-1}\right\}\right] .
\end{aligned}
$$


To prepare for the second renormalization group transformation, we rewrite this partition function in terms of updated coupling fields in the following way

$$
\begin{aligned}
Z= & \int D \Phi_{i} D \varphi_{i}^{(0)} D \rho_{i}^{(0)} D s_{i i+1}^{(0)} D t_{i i+1}^{(0)} D \chi_{i}^{(0)} D m_{i}^{2(0)} D v_{i}^{(0)} D u_{i}^{(0)} \\
& \times D \varphi_{i}^{(1)} D \rho_{i}^{(1)} D s_{i i+1}^{(1)} D t_{i i+1}^{(1)} D \chi_{i}^{(1)} D m_{i}^{2(1)} D v_{i}^{(1)} D u_{i}^{(1)} \\
& \times \exp \left[-\frac{1}{4} \sum_{i=1}^{M} \operatorname{tr}_{\tau \tau^{\prime}} \ln \left(-\partial_{\tau}^{2}+m_{i}^{2(0)}\right)\right. \\
& -\int_{0}^{\beta} d \tau \sum_{i=1}^{M}\left\{\left(\partial_{\tau} \Phi_{i}\right)^{2}+m_{i}^{2(1)} \Phi_{i}^{2}-i \varphi_{i}^{(0)} \Phi_{i}^{2}-t_{i i+1}^{(1)}\left(\Phi_{i} \Phi_{i+1}+\Phi_{i+1} \Phi_{i}\right)\right. \\
& +\frac{u_{i}^{(0)}}{2} \rho_{i}^{(0) 2}+i \varphi_{i}^{(0)} \rho_{i}^{(0)}+i s_{i i+1}^{(0)}\left(t_{i i+1}^{(0)}-t\right)+i \chi_{i}^{(0)}\left(m_{i}^{2(0)}-m^{2}\right)+i v_{i}^{(0)}\left(u_{i}^{(0)}-u\right) \\
& +\frac{u_{i}^{(1)}}{2}\left(\rho_{i}^{(1) 2}+3 \rho_{i}^{(1)} \rho_{i+1}^{(1)}\right) \\
& +i \varphi_{i}^{(1)}\left(\rho_{i}^{(1)}-\Phi_{i}^{2}\right)+i s_{i i+1}^{(1)}\left[t_{i i+1}^{(1)}-\frac{t_{i i+1}^{(0) 2}}{2 m_{i}^{4(0)}+u_{i}^{(0)}}\left\{m_{i}^{2(0)}-\frac{u_{i}^{(0)} t_{i i+1}^{(0) 2}}{m_{i}^{4(0)}}\left(\rho_{i}^{(1)}+\rho_{i+1}^{(1)}\right)\right\}\right] \\
& +i \chi_{i}^{(1)}\left\{m_{i}^{2(1)}-\left(m_{i}^{2(0)}-\frac{2 m_{i}^{2(0)} t_{i i+1}^{(0) 2}}{2 m_{i}^{4(0)}+u_{i}^{(0)}}\right)\right\} \\
& \left.\left.+i v_{i}^{(1)}\left(u_{i}^{(1)}-\frac{2 u_{i}^{(0)} t_{i i+1}^{(0) 4}}{m_{i}^{4(0)}\left(2 m_{i}^{4(0)}+u_{i}^{(0)}\right)}\right)+\frac{1}{16 m_{i}^{4(0)}}\left(\frac{1}{u_{i}^{(0)}}+\frac{1}{2 m_{i}^{4(0)}}\right)^{-1}\right\}\right] .
\end{aligned}
$$

Here, $s_{i i+1}^{(1)}, \chi_{i}^{(1)}$, and $v_{i}^{(1)}$ are Lagrange multiplier fields to impose the renormalization group flow equations for hopping, mass, and self-interaction fields, respectively. $\varphi_{i}^{(1)}$ is a Lagrange multiplier field to realize the Hubbard-Stratonovich transformation for newly generated effective interactions.

Shifting $\varphi_{i}^{(1)}$ as

$$
\varphi_{i}^{(1)} \Longrightarrow \varphi_{i}^{(1)}-\varphi_{i}^{(0)}
$$

and keeping only local interactions in the sense of the gradient expansion, we obtain

$$
\begin{aligned}
Z= & \int D \Phi_{i} D \varphi_{i}^{(0)} D \rho_{i}^{(0)} D s_{i i+1}^{(0)} D t_{i i+1}^{(0)} D \chi_{i}^{(0)} D m_{i}^{2(0)} D v_{i}^{(0)} D u_{i}^{(0)} \\
& \times D \varphi_{i}^{(1)} D \rho_{i}^{(1)} D s_{i i+1}^{(1)} D t_{i i+1}^{(1)} D \chi_{i}^{(1)} D m_{i}^{2(1)} D v_{i}^{(1)} D u_{i}^{(1)} \\
& \times \exp \left[-\frac{1}{4} \sum_{i=1}^{M} \operatorname{tr}_{\tau \tau^{\prime}} \ln \left(-\partial_{\tau}^{2}+m_{i}^{2(0)}\right)\right. \\
& -\int_{0}^{\beta} d \tau \sum_{i=1}^{M}\left\{\left(\partial_{\tau} \Phi_{i}\right)^{2}+m_{i}^{2(1)} \Phi_{i}^{2}-i \varphi_{i}^{(1)} \Phi_{i}^{2}-t_{i i+1}^{(1)}\left(\Phi_{i} \Phi_{i+1}+\Phi_{i+1} \Phi_{i}\right)\right. \\
& +\frac{u_{i}^{(0)}}{2} \rho_{i}^{(0) 2}+i \varphi_{i}^{(0)} \rho_{i}^{(0)}+i s_{i i+1}^{(0)}\left(t_{i i+1}^{(0)}-t\right)+i \chi_{i}^{(0)}\left(m_{i}^{2(0)}-m^{2}\right)+i v_{i}^{(0)}\left(u_{i}^{(0)}-u\right) \\
& +\frac{u_{i}^{(1)}}{2} \rho_{i}^{(1) 2}+i \rho_{i}^{(1)}\left(\varphi_{i}^{(1)}-\varphi_{i}^{(0)}\right)
\end{aligned}
$$




$$
\begin{aligned}
& +i s_{i i+1}^{(1)}\left\{t_{i i+1}^{(1)}-\frac{t_{i i+1}^{(0) 2}}{2 m_{i}^{4(0)}+u_{i}^{(0)}}\left(m_{i}^{2(0)}-\frac{2 u_{i}^{(0)} t_{i i+1}^{(0) 2}}{m_{i}^{4(0)}} \rho_{i}^{(1)}\right)\right\} \\
& +i \chi_{i}^{(1)}\left\{m_{i}^{2(1)}-\left(m_{i}^{2(0)}-\frac{2 m_{i}^{2(0)} t_{i i+1}^{(0) 2}}{2 m_{i}^{4(0)}+u_{i}^{(0)}}\right)\right\} \\
& \left.\left.+i v_{i}^{(1)}\left(u_{i}^{(1)}-\frac{u_{i}^{(0)} t_{i i+1}^{(0) 4}}{2 m_{i}^{4(0)}\left(2 m_{i}^{4(0)}+u_{i}^{(0)}\right)}\right)+\frac{1}{16 m_{i}^{4(0)}}\left(\frac{1}{u_{i}^{(0)}}+\frac{1}{2 m_{i}^{4(0)}}\right)^{-1}\right\}\right] .
\end{aligned}
$$

This completes the first renormalization group transformation.

It is straightforward to extend this expression in a recursive way, given by

$$
\begin{aligned}
& Z=\int D \Phi_{i} \Pi_{l=0}^{f} D \varphi_{i}^{(l)} D \rho_{i}^{(l)} D s_{i i+1}^{(l)} D t_{i i+1}^{(l)} D \chi_{i}^{(l)} D m_{i}^{2(l)} D v_{i}^{(l)} D u_{i}^{(l)} \\
& \exp \left[-\frac{1}{4} \sum_{l=1}^{f} \sum_{i=1}^{M} \operatorname{tr}_{\tau \tau^{\prime}} \ln \left(-\partial_{\tau}^{2}+m_{i}^{2(l-1)}\right)\right. \\
& -\int_{0}^{\beta} d \tau \sum_{i=1}^{M}\left\{\left(\partial_{\tau} \Phi_{i}\right)^{2}+m_{i}^{2(f)} \Phi_{i}^{2}-i \varphi_{i}^{(f)} \Phi_{i}^{2}-t_{i i+1}^{(f)}\left(\Phi_{i} \Phi_{i+1}+\Phi_{i+1} \Phi_{i}\right)\right. \\
& +\frac{u_{i}^{(0)}}{2} \rho_{i}^{(0) 2}+i \varphi_{i}^{(0)} \rho_{i}^{(0)}+i s_{i i+1}^{(0)}\left(t_{i i+1}^{(0)}-t\right)+i \chi_{i}^{(0)}\left(m_{i}^{2(0)}-m^{2}\right)+i v_{i}^{(0)}\left(u_{i}^{(0)}-u\right) \\
& +\sum_{l=1}^{f}\left[\frac{u_{i}^{(l)}}{2} \rho_{i}^{(l) 2}+i \rho_{i}^{(l)}\left(\varphi_{i}^{(l)}-\varphi_{i}^{(l-1)}\right)\right. \\
& +i s_{i i+1}^{(l)}\left\{t_{i i+1}^{(l)}-\frac{t_{i i+1}^{(l-1) 2}}{2 m_{i}^{4(l-1)}+u_{i}^{(l-1)}}\left(m_{i}^{2(l-1)}-\frac{2 u_{i}^{(l-1)} t_{i i+1}^{(l-1) 2}}{m_{i}^{4(l-1)}} \rho_{i}^{(l)}\right)\right\} \\
& +i \chi_{i}^{(l)}\left\{m_{i}^{2(l)}-\left(m_{i}^{2(l-1)}-\frac{2 m_{i}^{2(l-1)} t_{i i+1}^{(l-1) 2}}{2 m_{i}^{4(l-1)}+u_{i}^{(l-1)}}\right)\right\} \\
& \left.\left.\left.+i v_{i}^{(l)}\left(u_{i}^{(l)}-\frac{u_{i}^{(l-1)} t_{i i+1}^{(l-1) 4}}{2 m_{i}^{4(l-1)}\left(2 m_{i}^{4(l-1)}+u_{i}^{(l-1)}\right)}\right)+\frac{1}{16 m_{i}^{4(l-1)}}\left(\frac{1}{u_{i}^{(l-1)}}+\frac{1}{2 m_{i}^{4(l-1)}}\right)^{-1}\right]\right\}\right] .
\end{aligned}
$$

Resorting to

$$
a \sum_{l=1}^{f} \Longrightarrow \int_{0}^{z_{f}} d z
$$

and

$$
\frac{\varphi_{i}^{(l)}-\varphi_{i}^{(l-1)}}{a} \Longrightarrow \partial_{z} \varphi(i, \tau, z),
$$

where $a$ is a scale for the renormalization group transformation, we rewrite the above 
partition function in the following way

$$
\begin{aligned}
Z= & \int D \Phi(i, \tau) \int D \varphi(i, \tau, z) D \rho(i, \tau, z) \\
& \times \int D s(i, i+1, \tau, z) D t(i, i+1, \tau, z) D \chi(i, \tau, z) D m^{2}(i, \tau, z) D v(i, \tau, z) D u(i, \tau, z) \\
& \times \exp \left\{-S_{\mathrm{IR}}-S_{\mathrm{UV}}-S_{\mathrm{Bulk}}\right\} .
\end{aligned}
$$

Here, both IR and UV effective action are given by

$$
\begin{aligned}
S_{\mathrm{IR}}+S_{\mathrm{UV}}= & \int_{0}^{\beta} d \tau \sum_{i=1}^{M}\left\{\left(\partial_{\tau} \Phi(i, \tau)\right)^{2}+m^{2}\left(i, \tau, z_{f}\right)[\Phi(i, \tau)]^{2}-i \varphi\left(i, \tau, z_{f}\right)[\Phi(i, \tau)]^{2}\right. \\
& -t\left(i, i+1, \tau, z_{f}\right)(\Phi(i, \tau) \Phi(i+1, \tau)+\Phi(i+1, \tau) \Phi(i, \tau)) \\
& +\frac{u(i, \tau, 0)}{2}[\rho(i, \tau, 0)]^{2}+i \varphi(i, \tau, 0) \rho(i, \tau, 0) \\
& +i s(i, i+1, \tau, 0)(t(i, i+1, \tau, 0)-t)+i \chi(i, \tau, 0)\left(m^{2}(i, \tau, 0)-m^{2}\right) \\
& +i v(i, \tau, 0)(u(i, \tau, 0)-u)\}
\end{aligned}
$$

The bulk effective action is

$$
\begin{aligned}
S_{\mathrm{Bulk}}= & \int_{0}^{z_{f}} d z \int_{0}^{\beta} d \tau \sum_{i=1}^{M}\left\{\frac{u(i, \tau, z)}{2 a}[\rho(i, \tau, z)]^{2}+i \rho(i, \tau, z) \partial_{z} \varphi(i, \tau, z)\right. \\
& +i s(i, i+1, \tau, z)\left\{\partial_{z} t(i, i+1, \tau, z)+\frac{1}{a} t(i, i+1, \tau, z)\right. \\
& \left.-\frac{[t(i, i+1, \tau, z)]^{2}}{a\left(2\left[m^{2}(i, \tau, z)\right]^{2}+u(i, \tau, z)\right)}\left(m^{2}(i, \tau, z)-\frac{2 u(i, \tau, z)[t(i, i+1, \tau, z)]^{2}}{\left[m^{2}(i, \tau, z)\right]^{2}} \rho(i, \tau, z)\right)\right\} \\
& +i \chi(i, \tau, z)\left\{\partial_{z} m^{2}(i, \tau, z)+\frac{2 m^{2}(i, \tau, z)[t(i, i+1, \tau, z)]^{2}}{a\left(2\left[m^{2}(i, \tau, z)\right]^{2}+u(i, \tau, z)\right)}\right\} \\
& +i v(i, \tau, z)\left(\partial_{z} u(i, \tau, z)+\frac{1}{a} u(i, \tau, z)-\frac{u(i, \tau, z)[t(i, i+1, \tau, z)]^{4}}{2 a\left[m^{2}(i, \tau, z)\right]^{2}\left(2\left[m^{2}(i, \tau, z)\right]^{2}+u(i, \tau, z)\right)}\right) \\
& \left.+\frac{u(i, \tau, z)}{8 a\left(2\left[m^{2}(i, \tau, z)\right]^{2}+u(i, \tau, z)\right)}+\frac{1}{4 a} \ln \left(-\partial_{\tau}^{2}+m^{2}(i, \tau, z)\right)\right\} .
\end{aligned}
$$

Performing Gaussian integrals for $\rho(i, \tau, z), s(i, i+1, \tau, z), \chi(i, \tau, z)$, and $v(i, \tau, z)$, one can further simplify these expressions as

$$
\begin{aligned}
Z= & \int D \Phi(i, \tau) D \varphi(i, \tau, z) D t(i, i+1, \tau, z) D m^{2}(i, \tau, z) D u(i, \tau, z) \\
& \times \delta(t(i, i+1, \tau, 0)-t) \delta\left(m^{2}(i, \tau, 0)-m^{2}\right) \delta(u(i, \tau, 0)-u) \\
& \times \delta\left(\partial_{z} m^{2}(i, \tau, z)+\frac{2 m^{2}(i, \tau, z)[t(i, i+1, \tau, z)]^{2}}{a\left(2\left[m^{2}(i, \tau, z)\right]^{2}+u(i, \tau, z)\right)}\right)
\end{aligned}
$$




$$
\begin{aligned}
& \times \delta\left(\partial_{z} u(i, \tau, z)+\frac{1}{a} u(i, \tau, z)-\frac{u(i, \tau, z)[t(i, i+1, \tau, z)]^{4}}{2 a\left[m^{2}(i, \tau, z)\right]^{2}\left(2\left[m^{2}(i, \tau, z)\right]^{2}+u(i, \tau, z)\right)}\right) \\
& \times \exp \left\{-S_{\mathrm{IR}}-S_{\mathrm{UV}}-S_{\mathrm{Bulk}}\right\} .
\end{aligned}
$$

Both IR and UV effective actions are given by

$$
\begin{aligned}
S_{\mathrm{IR}}+S_{\mathrm{UV}}= & \int_{0}^{\beta} d \tau \sum_{i=1}^{M}\left\{\left(\partial_{\tau} \Phi(i, \tau)\right)^{2}+m^{2}\left(i, \tau, z_{f}\right)[\Phi(i, \tau)]^{2}-i \varphi\left(i, \tau, z_{f}\right)[\Phi(i, \tau)]^{2}\right. \\
& \left.-t\left(i, i+1, \tau, z_{f}\right)(\Phi(i, \tau) \Phi(i+1, \tau)+\Phi(i+1, \tau) \Phi(i, \tau))+\frac{1}{2 u(i, \tau, 0)}[\varphi(i, \tau, 0)]^{2}\right\}
\end{aligned}
$$

and the bulk effective action is

$$
\begin{aligned}
S_{\mathrm{Bulk}}= & \int_{0}^{z_{f}} d z \int_{0}^{\beta} d \tau \sum_{i=1}^{M}\left\{\frac{3 a}{8 u(i, \tau, z)}\left[\partial_{z} \varphi(i, \tau, z)\right]^{2}\right. \\
& +\frac{a\left[m^{2}(i, \tau, z)\right]^{4}\left(2\left[m^{2}(i, \tau, z)\right]^{2}+u(i, \tau, z)\right)^{2}}{8 u(i, \tau, z)[t(i, i+1, \tau, z)]^{8}} \\
& \times\left\{\partial_{z} t(i, i+1, \tau, z)+\frac{1}{a} t(i, i+1, \tau, z)-\frac{m^{2}(i, \tau, z)[t(i, i+1, \tau, z)]^{2}}{a\left(2\left[m^{2}(i, \tau, z)\right]^{2}+u(i, \tau, z)\right)}\right\}^{2} \\
& +\frac{a\left[m^{2}(i, \tau, z)\right]^{2}\left(2\left[m^{2}(i, \tau, z)\right]^{2}+u(i, \tau, z)\right)}{8 u(i, \tau, z)[t(i, i+1, \tau, z)]^{4}}\left(-i \partial_{z} \varphi(i, \tau, z)\right) \\
& \times\left\{\partial_{z} t(i, i+1, \tau, z)+\frac{1}{a} t(i, i+1, \tau, z)-\frac{m^{2}(i, \tau, z)[t(i, i+1, \tau, z)]^{2}}{a\left(2\left[m^{2}(i, \tau, z)\right]^{2}+u(i, \tau, z)\right)}\right\} \\
& \left.+\frac{u(i, \tau, z)}{8 a\left(2\left[m^{2}(i, \tau, z)\right]^{2}+u(i, \tau, z)\right)}+\frac{1}{4 a} \ln \left(-\partial_{\tau}^{2}+m^{2}(i, \tau, z)\right)\right\} .
\end{aligned}
$$

For the presentation and as a natural approximation, we take a uniform ansatz in the following way

$$
\begin{aligned}
Z= & \int D \Phi(i, \tau) D \varphi(z) D t(z) D m^{2}(z) D u(z) \delta(t(0)-t) \delta\left(m^{2}(0)-m^{2}\right) \delta(u(0)-u) \\
& \times \delta\left(\partial_{z} m^{2}(z)+\frac{2 m^{2}(z)[t(z)]^{2}}{a\left(2\left[m^{2}(z)\right]^{2}+u(z)\right)}\right) \\
& \times \delta\left(\partial_{z} u(z)+\frac{1}{a} u(z)-\frac{u(z)[t(z)]^{4}}{2 a\left[m^{2}(z)\right]^{2}\left(2\left[m^{2}(z)\right]^{2}+u(z)\right)}\right) \\
& \times \exp \left\{-S_{\mathrm{IR}}-S_{\mathrm{UV}}-S_{\mathrm{Bulk}}\right\},
\end{aligned}
$$


where the IR and UV effective actions are

$$
\begin{aligned}
S_{\mathrm{IR}}+S_{\mathrm{UV}}= & \int_{0}^{\beta} d \tau \sum_{i=1}^{M}\left\{\left(\partial_{\tau} \Phi(i, \tau)\right)^{2}+m^{2}\left(z_{f}\right)[\Phi(i, \tau)]^{2}-i \varphi\left(z_{f}\right)[\Phi(i, \tau)]^{2}\right. \\
& \left.-t\left(z_{f}\right)(\Phi(i, \tau) \Phi(i+1, \tau)+\Phi(i+1, \tau) \Phi(i, \tau))+\frac{1}{2 u(0)}[\varphi(0)]^{2}\right\}
\end{aligned}
$$

and the bulk effective action is

$$
\begin{aligned}
S_{\text {Bulk }}= & \beta M \int_{0}^{z_{f}} d z\left\{\frac{3 a}{8 u(z)}\left[\partial_{z} \varphi(z)\right]^{2}\right. \\
& +\frac{a\left[m^{2}(z)\right]^{4}\left(2\left[m^{2}(z)\right]^{2}+u(z)\right)^{2}}{8 u(z)[t(z)]^{8}}\left\{\partial_{z} t(z)+\frac{1}{a} t(z)-\frac{m^{2}(z)[t(z)]^{2}}{a\left(2\left[m^{2}(z)\right]^{2}+u(z)\right)}\right\}^{2} \\
& +\frac{a\left[m^{2}(z)\right]^{2}\left(2\left[m^{2}(z)\right]^{2}+u(z)\right)}{8 u(z)[t(z)]^{4}}\left(-i \partial_{z} \varphi(z)\right)\left\{\partial_{z} t(z)+\frac{1}{a} t(z)-\frac{m^{2}(z)[t(z)]^{2}}{a\left(2\left[m^{2}(z)\right]^{2}+u(z)\right)}\right\} \\
& \left.+\frac{u(z)}{8 a\left(2\left[m^{2}(z)\right]^{2}+u(z)\right)}+\frac{1}{4 a} \frac{1}{\beta} \sum_{i \omega_{n}} \ln \left(\omega_{n}^{2}+m^{2}(z)\right)\right\} .
\end{aligned}
$$

Finally, we perform the mean-field analysis, where quantum fluctuations of $\varphi(z)$ and $t(z)$ are neglected to be their saddle-point values. Considering

$$
\varphi(z) \Longrightarrow-i \varphi(z)
$$

for real saddle-point values in $\varphi(z)$, we obtain the mean-field free-energy functional for $\varphi(z)$ and $t(z)$

$$
\begin{aligned}
& \frac{1}{M} F\left[\varphi(z), t(z) ; z_{f}\right]= \\
& \frac{1}{2 \beta} \sum_{i \omega_{n}} \frac{1}{M} \sum_{q} \ln \left\{\omega_{n}^{2}-2 t\left(z_{f}\right) \cos q+m^{2}\left(z_{f}\right)-\varphi\left(z_{f}\right)\right\}-\frac{1}{2 u(0)}[\varphi(0)]^{2} \\
& +\int_{0}^{z_{f}} d z\left\{-\frac{3 a}{8 u(z)}\left[\partial_{z} \varphi(z)\right]^{2}\right. \\
& +\frac{a\left[m^{2}(z)\right]^{4}\left(2\left[m^{2}(z)\right]^{2}+u(z)\right)^{2}}{8 u(z)[t(z)]^{8}}\left\{\partial_{z} t(z)+\frac{1}{a} t(z)-\frac{m^{2}(z)[t(z)]^{2}}{a\left(2\left[m^{2}(z)\right]^{2}+u(z)\right)}\right\}^{2} \\
& -\frac{a\left[m^{2}(z)\right]^{2}\left(2\left[m^{2}(z)\right]^{2}+u(z)\right)}{8 u(z)[t(z)]^{4}}\left(\partial_{z} \varphi(z)\right)\left\{\partial_{z} t(z)+\frac{1}{a} t(z)-\frac{m^{2}(z)[t(z)]^{2}}{a\left(2\left[m^{2}(z)\right]^{2}+u(z)\right)}\right\} \\
& \left.+\frac{u(z)}{8 a\left(2\left[m^{2}(z)\right]^{2}+u(z)\right)}+\frac{1}{4 a} \frac{1}{\beta} \sum_{i \omega_{n}} \ln \left(\omega_{n}^{2}+m^{2}(z)\right)\right\} .
\end{aligned}
$$


The renormalization group equation for the mass parameter is

$$
\partial_{z} m^{2}(z)=-\frac{2 m^{2}(z)[t(z)]^{2}}{a\left(2\left[m^{2}(z)\right]^{2}+u(z)\right)}
$$

and that for the interaction vertex is

$$
\partial_{z} u(z)=-\frac{1}{a} u(z)+\frac{u(z)[t(z)]^{4}}{2 a\left[m^{2}(z)\right]^{2}\left(2\left[m^{2}(z)\right]^{2}+u(z)\right)} .
$$

Minimizing this free-energy functional with respect to both $\varphi(z)$ and $t(z)$, we obtain their equations of motion, given by the second-order derivatives in the coordinate of the extra dimensional space. On the other hand, their IR boundary conditions are given by the linear derivatives in $z$, corresponding to the renormalization group equation for the hopping integral and the Callan-Symanzik equation for the order parameter field, respectively.

\section{B Further developments for the invariance of entanglement entropy with respect to renormalization group transformations}

\section{B.1 An identity for entanglement entropy at $z=z_{f}$}

Resorting to the evolution equation for the metric tensor along the extra-dimensional space, we rewrite the area law of the matter sector at $z=z_{f}$ in terms of the Green's function as follows

$$
\begin{aligned}
\partial_{z_{f}} \mathcal{A}\left[\Sigma\left(z_{f}\right)\right] & =\int_{\Sigma\left(z_{f}\right)} d^{D-2} x_{\perp} \partial_{z_{f}} \sqrt{\gamma\left(x, z_{f}\right)} \\
& =\frac{1}{2} \int_{\Sigma\left(z_{f}\right)} d^{D-2} x_{\perp} \sqrt{\gamma\left(x, z_{f}\right)} \gamma^{i j}\left(x, z_{f}\right) \partial_{z_{f}} \gamma_{i j}\left(x, z_{f}\right) \\
& =-\frac{1}{4 d z} \int_{\Sigma\left(z_{f}\right)} d^{D-2} x_{\perp} \sqrt{\gamma\left(x, z_{f}\right)} \gamma^{i j}\left(x, z_{f}\right)\left(\partial_{i} \partial_{j} G\left[x, x ; g_{\mu \nu}\left(x, z_{f}\right)\right]\right) .
\end{aligned}
$$

We recall that the Green's function is given by

$$
\begin{aligned}
& \left\{-\frac{1}{\sqrt{g\left(x, z_{f}\right)}} \partial_{\mu}\left(\sqrt{g\left(x, z_{f}\right)} g^{\mu \nu}\left(x, z_{f}\right) \partial_{\nu}\right)+\frac{m^{2}}{e^{2 d z}-1}\right\} G\left[x, x^{\prime} ; g_{\mu \nu}\left(x, z_{f}\right)\right] \\
& =\frac{1}{\sqrt{g\left(x, z_{f}\right)}} \delta^{(D)}\left(x-x^{\prime}\right) .
\end{aligned}
$$

One can reformulate this Green's function in terms of the geometric information such as metric and curvature, considering the heat kernel expansion. Introducing the heat kernel $K\left(s, x, x^{\prime} ; z\right) \equiv\left\langle x z\left|e^{-s \mathcal{D}}\right| x^{\prime} z\right\rangle$ with the differential operator $\mathcal{D} \equiv$ $-\frac{1}{\sqrt{g(x, z)}} \partial_{\mu}\left(\sqrt{g(x, z)} g^{\mu \nu}(x, z) \partial_{\nu}\right)+\frac{m^{2}}{e^{2 d z}-1}$, which satisfies the diffusion equation $\left(\partial_{s}+\right.$ $\mathcal{D}) K\left(s, x, x^{\prime} ; z\right)=0$ with an initial condition $K\left(s=0, x, x^{\prime} ; z\right)=\frac{1}{\sqrt{g(x, z)}} \delta^{(D)}\left(x-x^{\prime}\right)$, we obtain

$$
G\left(x, x^{\prime} ; z\right)=\int_{\epsilon^{2}}^{\infty} d s K\left(s, x, x^{\prime} ; z\right)
$$


Here, $\epsilon$ is a UV cutoff to cure the UV divergence of the entanglement entropy, introduced in subsection 5.2. As discussed in the heat-kernel expansion eq. (5.14), we express the Green's function in terms of the metric and curvature at $z=z_{f}$ as

$$
\begin{aligned}
G\left(x, x ; z_{f}\right)= & \int_{\epsilon^{2}}^{\infty} d s K\left(s, x, x ; z_{f}\right)=\int_{\epsilon^{2}}^{\infty} d s \sum_{n=0}^{\infty} a_{n}\left(x, z_{f}\right) s^{\frac{n-D}{2}} \\
= & \int_{\epsilon^{2}}^{\infty} d s\left\{a_{0}\left(x, z_{f}\right) s^{-\frac{D}{2}}+a_{2}\left(x, z_{f}\right) s^{\frac{2-D}{2}}+a_{4}\left(x, z_{f}\right) s^{\frac{4-D}{2}}+\ldots\right\} \\
= & \int_{\epsilon^{2}}^{\infty} d s \frac{1}{(4 \pi)^{\frac{D}{2}}}\left[s^{-\frac{D}{2}}+\left(\frac{1}{6} R\left(x, z_{f}\right)-\frac{m^{2}}{e^{2 d z}-1}\right) s^{\frac{2-D}{2}}\right. \\
& +\left\{\frac{1}{180} R_{\mu \nu \alpha \beta}^{2}\left(x, z_{f}\right)-\frac{1}{180} R_{\mu \nu}^{2}\left(x, z_{f}\right)\right. \\
& +\frac{1}{6} \frac{1}{\sqrt{g\left(x, z_{f}\right)}} \partial_{\mu}\left(\sqrt{g\left(x, z_{f}\right)} g^{\mu \nu}\left(x, z_{f}\right) \partial_{\nu}\right)\left(\frac{1}{5} R\left(x, z_{f}\right)-\frac{m^{2}}{e^{2 d z}-1}\right) \\
& \left.\left.+\frac{1}{2}\left(\frac{1}{6} R\left(x, z_{f}\right)-\frac{m^{2}}{e^{2 d z}-1}\right)^{2}\right\} s^{\frac{4-D}{2}}+\ldots\right] .
\end{aligned}
$$

Inserting this expression into eq. (B.1), we obtain

$$
\begin{aligned}
& \partial_{z_{f}} \mathcal{A}\left[\Sigma\left(z_{f}\right)\right]= \\
& -\frac{1}{4 d z}\left(\frac{1}{(4 \pi)^{\frac{D}{2}}} \int_{\epsilon^{2}}^{\infty} d s s^{\frac{2-D}{2}}\right) \int_{\Sigma\left(z_{f}\right)} d^{D-2} x_{\perp} \sqrt{\gamma\left(x, z_{f}\right)} \gamma^{i j}\left(x, z_{f}\right) \partial_{i} \partial_{j}\left(\frac{1}{6} R\left(x, z_{f}\right)-\frac{m^{2}}{e^{2 d z}-1}\right) \\
& -\frac{1}{4 d z}\left(\frac{1}{(4 \pi)^{\frac{D}{2}}} \int_{\epsilon^{2}}^{\infty} d s s^{\frac{4-D}{2}}\right) \int_{\Sigma\left(z_{f}\right)} d^{D-2} x_{\perp} \sqrt{\gamma\left(x, z_{f}\right)} \gamma^{i j}\left(x, z_{f}\right) \\
& \times \partial_{i} \partial_{j}\left\{\frac{1}{180} R_{\mu \nu \alpha \beta}^{2}\left(x, z_{f}\right)-\frac{1}{180} R_{\mu \nu}^{2}\left(x, z_{f}\right)\right. \\
& +\frac{1}{6} \frac{1}{\sqrt{g\left(x, z_{f}\right)}} \partial_{\mu}\left(\sqrt{g\left(x, z_{f}\right)} g^{\mu \nu}\left(x, z_{f}\right) \partial_{\nu}\right)\left(\frac{1}{5} R\left(x, z_{f}\right)-\frac{m^{2}}{e^{2 d z}-1}\right) \\
& \left.+\frac{1}{2}\left(\frac{1}{6} R\left(x, z_{f}\right)-\frac{m^{2}}{e^{2 d z}-1}\right)^{2}\right\} .
\end{aligned}
$$

Combining this expression with that of the gravity sector, we reach the following identity for the entanglement entropy

$$
\begin{aligned}
& \frac{N}{6(D-2)(4 \pi)^{\frac{D}{2}-1}} \frac{1}{\epsilon^{D-2}} \frac{1}{4 d z} \frac{1}{(4 \pi)^{\frac{D}{2}}} \int_{\Sigma\left(z_{f}\right)} d^{D-2} x_{\perp} \sqrt{\gamma\left(x, z_{f}\right)} \gamma^{i j}\left(x, z_{f}\right)[ \\
& -\left(\int_{\epsilon^{2}}^{\infty} d s s^{\frac{2-D}{2}}\right) \partial_{i} \partial_{j}\left(\frac{1}{6} R\left(x, z_{f}\right)-\frac{m^{2}}{e^{2 d z}-1}\right) \\
& -\left(\int_{\epsilon^{2}}^{\infty} d s s^{\frac{4-D}{2}}\right) \partial_{i} \partial_{j}\left\{\frac{1}{180} R_{\mu \nu \alpha \beta}^{2}\left(x, z_{f}\right)-\frac{1}{180} R_{\mu \nu}^{2}\left(x, z_{f}\right)\right.
\end{aligned}
$$




$$
\begin{aligned}
& +\frac{1}{6} \frac{1}{\sqrt{g\left(x, z_{f}\right)}} \partial_{\mu}\left(\sqrt{g\left(x, z_{f}\right)} g^{\mu \nu}\left(x, z_{f}\right) \partial_{\nu}\right)\left(\frac{1}{5} R\left(x, z_{f}\right)-\frac{m^{2}}{e^{2 d z}-1}\right) \\
& \left.\left.+\frac{1}{2}\left(\frac{1}{6} R\left(x, z_{f}\right)-\frac{m^{2}}{e^{2 d z}-1}\right)^{2}\right\}\right] \\
& +N \int d^{D-2} x_{\perp} \int_{0}^{2 \pi} d \theta \int_{0}^{\infty} d r \sqrt{g_{n}\left(r, x_{\perp}, z_{f}\right)}\left(T_{\mu \nu, n}^{\varphi}\left(r, x_{\perp}, z_{f}\right)+T_{\mu \nu, n}^{g_{\mu \nu}}\left(r, x_{\perp}, z_{f}\right)\right) \\
& \times\left.\frac{\partial g_{n}^{\mu \nu}\left(r, x_{\perp}, z_{f}\right)}{\partial n}\right|_{n=1}=0 .
\end{aligned}
$$

We point out that both integrals of $\int_{\epsilon^{2}}^{\infty} d s s^{(2-D) / 2}$ and $\int_{\epsilon^{2}}^{\infty} d s s^{(4-D) / 2}$ diverge for large $s$. Existence of these IR divergences may be an artifact of the small $s$-expansion. One may introduce the correlation length into the upper cutoff of these integrals as $\int_{\epsilon^{2}}^{\xi^{2}} d s s^{(2-D) / 2}$ and $\int_{\epsilon^{2}}^{\xi^{2}} d s s^{(4-D) / 2}$. We recall that this IR cutoff reproduces the entanglement entropy in $D=2$, as shown in eq. (5.21). Now, the identity is expressed in a fully geometric way.

\section{B.2 Entanglement entropy after the first iteration}

While it is written in terms of all the geometrical information, it is not clear how to interpret the above lengthy expression. In addition, it is not easy to check out this identity in a brute force way. In this respect we consider the entanglement entropy after the first iteration of the renormalization group transformation and verify the invariance explicitly for the case without all types of interactions.

We recall the partition function after the renormalization group transformation

$$
\begin{aligned}
Z^{(1)}= & \int D \phi_{\alpha} D g_{\mu \nu}^{(1)} \delta\left(g^{(1) \mu \nu}-g_{B}^{\mu \nu}-g_{B}^{\mu \nu^{\prime}}\left(\partial_{\nu^{\prime}} \partial_{\mu^{\prime}} G_{x x^{\prime}}\right)_{x^{\prime} \rightarrow x} g_{B}^{\mu^{\prime} \nu}\right) \\
& \times \exp \left[-\int d^{D} x \sqrt{g^{(1)}}\left\{g^{(1) \mu \nu}\left(\partial_{\mu} \phi_{\alpha}\right)\left(\partial_{\nu} \phi_{\alpha}\right)+m^{2} \phi_{\alpha}^{2}\right\}-\frac{N}{2 \kappa} \int d^{D} x \sqrt{g_{B}}\left(R_{B}-2 \Lambda\right)\right],
\end{aligned}
$$

where the coefficients of the gravity action are $2 d z \mathcal{C}_{R}=\frac{1}{2 \kappa}$ and $\frac{\mathcal{C}_{\Lambda}}{\mathcal{C}_{R}}=2 \Lambda$. Then, the entanglement entropy is

$$
\begin{aligned}
\mathcal{S}_{E E}^{(1)}= & \frac{N}{6(D-2)(4 \pi)^{\frac{D}{2}-1}} \frac{1}{\epsilon^{D-2}} \int_{\Sigma^{(1)}} d^{D-2} x_{\perp} \sqrt{g_{\perp}^{(1)}(x)} \\
& +\frac{N}{2 \kappa} \int d^{D-2} x_{\perp} \int_{0}^{2 \pi} d \theta \int_{0}^{\infty} d r \sqrt{g_{B, n}(x)}\left[2\left(R_{\mu \nu, n}^{B}(x)-\frac{1}{2} g_{\mu \nu, n}^{B}(x) R_{B, n}(x)+\Lambda g_{\mu \nu, n}^{B}(x)\right)\right. \\
& \left.+\frac{1}{g_{B, n}(x)} g_{\mu \mu^{\prime}, n}^{B}(x) g_{\nu \nu^{\prime}, n}^{B}(x) \partial_{\mu^{\prime \prime}} \partial_{\nu^{\prime \prime}}\left\{g_{B, n}(x)\left(g_{B, n}^{\mu^{\prime} \nu^{\prime}}(x) g_{B, n}^{\mu^{\prime \prime} \nu^{\prime \prime}}(x)-g_{B, n}^{\mu^{\prime} \mu^{\prime \prime}}(x) g_{B, n}^{\nu^{\prime} \nu^{\prime \prime}}(x)\right)\right\}\right] \\
& \times\left.\frac{\partial g_{B, n}^{\mu \nu}\left(r, x_{\perp}\right)}{\partial n}\right|_{n=1} .
\end{aligned}
$$

This entanglement entropy has to be identified with

$$
\mathcal{S}_{E E}=\frac{N}{6(D-2)(4 \pi)^{\frac{D}{2}-1}} \frac{1}{\epsilon^{D-2}} \int_{\Sigma_{B}} d^{D-2} x_{\perp} \sqrt{g_{\perp}^{B}(x)} .
$$


In other words, we have

$$
\mathcal{S}_{E E}=\mathcal{S}_{E E}^{(1)}
$$

As a result, we have to obtain

$$
\begin{aligned}
& \frac{N}{6(D-2)(4 \pi)^{\frac{D}{2}-1}} \frac{1}{\epsilon^{D-2}}\left(\int_{\Sigma_{B}} d^{D-2} x_{\perp} \sqrt{g_{\perp}^{B}(x)}-\int_{\Sigma^{(1)}} d^{D-2} x_{\perp} \sqrt{g_{\perp}^{(1)}(x)}\right) \\
& =\frac{N}{2 \kappa} \int d^{D-2} x_{\perp} \int_{0}^{2 \pi} d \theta \int_{0}^{\infty} d r \sqrt{g_{B, n}(x)}\left[2\left(R_{\mu \nu, n}^{B}(x)-\frac{1}{2} g_{\mu \nu, n}^{B}(x) R_{B, n}(x)+\Lambda g_{\mu \nu, n}^{B}(x)\right)\right. \\
& \quad+\frac{1}{g_{B, n}(x)} g_{\mu \mu^{\prime}, n}^{B}(x) g_{\nu \nu^{\prime}, n}^{B}(x) \partial_{\mu^{\prime \prime}} \partial_{\nu^{\prime \prime}} \\
& \left.\quad \times\left\{g_{B, n}(x)\left(g_{B, n}^{\mu^{\prime} \nu^{\prime}}(x) g_{B, n}^{\mu^{\prime \prime} \nu^{\prime \prime}}(x)-g_{B, n}^{\mu^{\prime} \mu^{\prime \prime}}(x) g_{B, n}^{\nu^{\prime} \nu^{\prime \prime}}(x)\right)\right\}\right]\left.\frac{\partial g_{B, n}^{\mu \nu}\left(r, x_{\perp}\right)}{\partial n}\right|_{n=1} .
\end{aligned}
$$

Recalling the evolution equation of

$$
g^{(1) \mu \nu}(x)=g_{B}^{\mu \nu}(x)+g_{B}^{\mu \nu^{\prime}}(x)\left(\partial_{\nu^{\prime}} \partial_{\mu^{\prime}} G_{x x^{\prime}}\right)_{x^{\prime} \rightarrow x} g_{B}^{\mu^{\prime} \nu}(x),
$$

we obtain

$$
\sqrt{g^{(1)}(x)}=\sqrt{g_{B}(x)}\left\{1-\frac{1}{2} g_{B}^{\mu \nu}(x)\left(\partial_{\mu} \partial_{\nu} G_{x x^{\prime}}\right)_{x^{\prime} \rightarrow x}\right\} .
$$

As a result, we have

$$
\begin{aligned}
& \int_{\Sigma_{B}} d^{D-2} x_{\perp} \sqrt{g_{\perp}^{B}(x)}-\int_{\Sigma^{(1)}} d^{D-2} x_{\perp} \sqrt{g_{\perp}^{(1)}(x)} \\
& \quad=\frac{1}{2} \int_{\Sigma_{B}} d^{D-2} x_{\perp} \sqrt{g_{\perp}^{B}(x)} g_{B \perp}^{\mu \nu}(x)\left(\partial_{\mu} \partial_{\nu} G_{x x^{\prime}}\right)_{x^{\prime} \rightarrow x} .
\end{aligned}
$$

The final equation to be verified is

$$
\begin{aligned}
& \frac{N}{6(D-2)(4 \pi)^{\frac{D}{2}-1}} \frac{1}{2 \epsilon^{D-2}} \int_{\Sigma_{B}} d^{D-2} x_{\perp} \sqrt{g_{\perp}^{B}(x)} g_{B \perp}^{\mu \nu}(x)\left(\partial_{\mu} \partial_{\nu} G_{x x^{\prime}}\right)_{x^{\prime} \rightarrow x} \\
& =\frac{N}{2 \kappa} \int d^{D-2} x_{\perp} \int_{0}^{2 \pi} d \theta \int_{0}^{\infty} d r \sqrt{g_{B, n}(x)}\left[2\left(R_{\mu \nu, n}^{B}(x)-\frac{1}{2} g_{\mu \nu, n}^{B}(x) R_{B, n}(x)+\Lambda g_{\mu \nu, n}^{B}(x)\right)\right. \\
& \quad+\frac{1}{g_{B, n}(x)} g_{\mu \mu^{\prime}, n}^{B}(x) g_{\nu \nu^{\prime}, n}^{B}(x) \partial_{\mu^{\prime \prime}} \partial_{\nu^{\prime \prime}} \\
& \left.\quad \times\left\{g_{B, n}(x)\left(g_{B, n}^{\mu^{\prime} \nu^{\prime}}(x) g_{B, n}^{\mu^{\prime \prime} \nu^{\prime \prime}}(x)-g_{B, n}^{\mu^{\prime} \mu^{\prime \prime}}(x) g_{B, n}^{\nu^{\prime} \nu^{\prime \prime}}(x)\right)\right\}\right]\left.\frac{\partial g_{B, n}^{\mu \nu}\left(r, x_{\perp}\right)}{\partial n}\right|_{n=1} .
\end{aligned}
$$

Here, the background metric is

$$
d s^{2}=d r^{2}+r^{2} d \theta^{2}+\delta_{i j} d x_{\perp}^{i} d x_{\perp}^{j}, \quad g_{r r}^{B}=1, \quad g_{\theta \theta}^{B}=r^{2}, \quad g_{i j}^{B \perp}=\delta_{i j}, \quad \sqrt{g_{B}}=r
$$

with

$$
\left.\frac{\partial g_{B n}^{\mu \nu}\left(r, x_{\perp}\right)}{\partial n}\right|_{n=1}=2 r^{2} \delta_{\mu \theta} \delta_{\nu \theta}
$$


and the Green's function is $[34,35]$

$$
\begin{aligned}
G_{x x^{\prime}}^{n} & \equiv G_{n}\left(r, r^{\prime}, \theta, \theta^{\prime}, x_{\perp}-x_{\perp}^{\prime}\right) \\
& =\int \frac{d^{D-2} p_{\perp}}{(2 \pi)^{D-2}} e^{i p_{\perp}\left(x_{\perp}-x_{\perp}^{\prime}\right)} \frac{1}{2 \pi n} \sum_{k=0}^{\infty} d_{k} \int_{0}^{\infty} d \lambda \lambda \frac{J_{k / n}(\lambda r) J_{k / n}\left(\lambda r^{\prime}\right)}{p_{\perp}^{2}+\lambda^{2}+m^{2} /\left(e^{2 d z}-1\right)} \cos \frac{k}{n}\left(\theta-\theta^{\prime}\right)
\end{aligned}
$$

with

$$
d_{0}=1, \quad d_{k>0}=2 .
$$

Inserting this Green's function with $n=1$ into eq. (B.15), the left-hand-side of this equation is written in terms of $\epsilon$ and $m$ with $\Sigma_{B}$. On the other hand, the right-hand-side of this equation is given by $\kappa$ and $\Lambda$ with a UV cutoff in the $r$ integral. As a result, renormalizing both coefficients of $\kappa$ and $\Lambda$ appropriately, one can verify this identity.

Open Access. This article is distributed under the terms of the Creative Commons Attribution License (CC-BY 4.0), which permits any use, distribution and reproduction in any medium, provided the original author(s) and source are credited.

\section{References}

[1] M.E. Peskin and D.V. Schroeder, An Introduction to quantum field theory, Westview, Boulder U.S.A. (1995).

[2] J.M. Maldacena, The Large $N$ limit of superconformal field theories and supergravity, Adv. Theor. Math. Phys. 2 (1998) 231 [hep-th/9711200] [INSPIRE].

[3] S.S. Gubser, I.R. Klebanov and A.M. Polyakov, Gauge theory correlators from noncritical string theory, Phys. Lett. B 428 (1998) 105 [hep-th/9802109] [InSPIRE].

[4] E. Witten, Anti-de Sitter space and holography, Adv. Theor. Math. Phys. 2 (1998) 253 [hep-th/9802150] [INSPIRE].

[5] M. Bianchi, D.Z. Freedman and K. Skenderis, Holographic renormalization, Nucl. Phys. B 631 (2002) 159 [hep-th/0112119] [INSPIRE].

[6] J. de Boer, E.P. Verlinde and H.L. Verlinde, On the holographic renormalization group, JHEP 08 (2000) 003 [hep-th/9912012] [INSPIRE].

[7] E.P. Verlinde and H.L. Verlinde, RG flow, gravity and the cosmological constant, JHEP 05 (2000) 034 [hep-th/9912018] [INSPIRE].

[8] O. Aharony, S.S. Gubser, J.M. Maldacena, H. Ooguri and Y. Oz, Large N field theories, string theory and gravity, Phys. Rept. 323 (2000) 183 [hep-th/9905111] [INSPIRE].

[9] M. Nozaki, S. Ryu and T. Takayanagi, Holographic Geometry of Entanglement Renormalization in Quantum Field Theories, JHEP 10 (2012) 193 [arXiv:1208.3469] [INSPIRE].

[10] I. Heemskerk, J. Penedones, J. Polchinski and J. Sully, Holography from Conformal Field Theory, JHEP 10 (2009) 079 [arXiv:0907.0151] [INSPIRE]. 
[11] I. Heemskerk and J. Polchinski, Holographic and Wilsonian Renormalization Groups, JHEP 06 (2011) 031 [arXiv: 1010.1264] [INSPIRE].

[12] T. Faulkner, H. Liu and M. Rangamani, Integrating out geometry: Holographic Wilsonian $R G$ and the membrane paradigm, JHEP 08 (2011) 051 [arXiv:1010.4036] [INSPIRE].

[13] R. de Mello Koch, A. Jevicki, K. Jin and J.P. Rodrigues, $A d S_{4} / C F T_{3}$ Construction from Collective Fields, Phys. Rev. D 83 (2011) 025006 [arXiv: 1008.0633] [InSPIRE].

[14] M.R. Douglas, L. Mazzucato and S.S. Razamat, Holographic dual of free field theory, Phys. Rev. D 83 (2011) 071701 [arXiv: 1011.4926] [InSPIRE].

[15] R.G. Leigh, O. Parrikar and A.B. Weiss, Holographic geometry of the renormalization group and higher spin symmetries, Phys. Rev. D 89 (2014) 106012 [arXiv:1402.1430] [InSPIRE].

[16] E. Mintun and J. Polchinski, Higher Spin Holography, RG, and the Light Cone, arXiv:1411.3151 [INSPIRE].

[17] F. Pastawski, B. Yoshida, D. Harlow and J. Preskill, Holographic quantum error-correcting codes: Toy models for the bulk/boundary correspondence, JHEP 06 (2015) 149 [arXiv: 1503.06237] [INSPIRE].

[18] X. Wen, G.Y. Cho, P.L.S. Lopes, Y. Gu, X.-L. Qi and S. Ryu, Holographic Entanglement Renormalization of Topological Insulators, Phys. Rev. B 94 (2016) 075124 [arXiv: 1605.07199] [INSPIRE].

[19] Y. Gu, C.H. Lee, X. Wen, G.Y. Cho, S. Ryu and X.-L. Qi, Holographic duality between $(2+1)$-dimensional quantum anomalous Hall state and $(3+1)$-dimensional topological insulators, Phys. Rev. B 94 (2016) 125107 [arXiv: 1605.00570] [INSPIRE].

[20] T. Faulkner, M. Guica, T. Hartman, R.C. Myers and M. Van Raamsdonk, Gravitation from Entanglement in Holographic CFTs, JHEP 03 (2014) 051 [arXiv:1312.7856] [INSPIRE].

[21] T. Faulkner, F.M. Haehl, E. Hijano, O. Parrikar, C. Rabideau and M. Van Raamsdonk, Nonlinear Gravity from Entanglement in Conformal Field Theories, JHEP 08 (2017) 057 [arXiv: 1705.03026] [INSPIRE].

[22] B. Czech, Einstein Equations from Varying Complexity, Phys. Rev. Lett. 120 (2018) 031601 [arXiv: 1706.00965] [INSPIRE].

[23] K.-S. Kim and C. Park, Emergent geometry from field theory: Wilson's renormalization group revisited, Phys. Rev. D 93 (2016) 121702 [arXiv: 1604.04990] [INSPIRE].

[24] G. Vidal, Entanglement Renormalization, Phys. Rev. Lett. 99 (2007) 220405 [cond-mat/0512165] [INSPIRE].

[25] J. Haegeman, T.J. Osborne, H. Verschelde and F. Verstraete, Entanglement Renormalization for Quantum Fields in Real Space, Phys. Rev. Lett. 110 (2013) 100402 [arXiv:1102.5524] [INSPIRE].

[26] G. Vidal, Understanding Quantum Phase Transitions, L.D. Carr eds., Taylor \& Francis, Boca Raton U.S.A. (2010).

[27] G. Evenbly and G. Vidal, Springer Series in Solid-State Sciences. Vol. 176: Strongly Correlated Systems. Numerical Methods, A. Avella and F. Mancini eds., Springer-Verlag, Heidelberg Germany (2013).

[28] G. Evenbly and G. Vidal, Tensor network states and geometry, J. Stat. Phys. 145 (2011) 891 [arXiv: 1106.1082v1]. 
[29] B. Swingle, Entanglement Renormalization and Holography, Phys. Rev. D 86 (2012) 065007 [arXiv: 0905.1317] [INSPIRE].

[30] J. Polchinski, Renormalization and Effective Lagrangians, Nucl. Phys. B 231 (1984) 269 [INSPIRE].

[31] S.-S. Lee, Background independent holographic description: From matrix field theory to quantum gravity, JHEP 10 (2012) 160 [arXiv:1204.1780] [INSPIRE].

[32] S.-S. Lee, Quantum Renormalization Group and Holography, JHEP 01 (2014) 076 [arXiv: 1305.3908] [INSPIRE].

[33] P. Lunts, S. Bhattacharjee, J. Miller, E. Schnetter, Y.B. Kim and S.-S. Lee, Ab initio holography, JHEP 08 (2015) 107 [arXiv: 1503.06474] [inSPIRE].

[34] P. Calabrese and J.L. Cardy, Entanglement entropy and quantum field theory, J. Stat. Mech. 0406 (2004) P06002 [hep-th/0405152] [InSPIRE].

[35] P. Calabrese and J. Cardy, Entanglement entropy and conformal field theory, J. Phys. A 42 (2009) 504005 [arXiv:0905.4013] [INSPIRE].

[36] S. Ryu and T. Takayanagi, Holographic derivation of entanglement entropy from AdS/CFT, Phys. Rev. Lett. 96 (2006) 181602 [hep-th/0603001] [INSPIRE].

[37] S. Ryu and T. Takayanagi, Aspects of Holographic Entanglement Entropy, JHEP 08 (2006) 045 [hep-th/0605073] [INSPIRE].

[38] J. Eisert, M. Cramer and M.B. Plenio, Area laws for the entanglement entropy - a review, Rev. Mod. Phys. 82 (2010) 277 [arXiv:0808.3773] [InSPIRE].

[39] T. Nishioka, Entanglement entropy: holography and renormalization group, Rev. Mod. Phys. 90 (2018) 035007 [arXiv: 1801.10352] [INSPIRE].

[40] N.D. Birrell and P.C.W. Davies, Quantum Fields in Curved Space, Cambridge University Press, New York U.S.A. (1982).

[41] J. Cardy, The TT deformation of quantum field theory as random geometry, JHEP 10 (2018) 186 [arXiv: 1801.06895] [INSPIRE].

[42] P.M. Chaikin and T.C. Lubensky, Principles of Condensed Matter Physics, Cambridge University Press, New York U.S.A. (1995).

[43] A.D. Sakharov, Vacuum quantum fluctuations in curved space and the theory of gravitation, Sov. Phys. Dokl. 12 (1968) 1040 [Gen. Rel. Grav. 32 (2000) 365]. [InSPIRE].

[44] M. Visser, Sakharov's induced gravity: A Modern perspective, Mod. Phys. Lett. A 17 (2002) 977 [gr-qc/0204062] [INSPIRE].

[45] J.W. York, Jr., Role of conformal three geometry in the dynamics of gravitation, Phys. Rev. Lett. 28 (1972) 1082 [INSPIRE].

[46] G.W. Gibbons and S.W. Hawking, Action Integrals and Partition Functions in Quantum Gravity, Phys. Rev. D 15 (1977) 2752 [InSPIRE].

[47] S.N. Solodukhin, Entanglement entropy of black holes, Living Rev. Rel. 14 (2011) 8 [arXiv: 1104.3712] [INSPIRE].

[48] K.-S. Kim, M. Park, J. Cho and C. Park, Emergent geometric description for a topological phase transition in the Kitaev superconductor model, Phys. Rev. D 96 (2017) 086015 [arXiv: 1610.07312] [INSPIRE]. 
[49] S.-S. Lee, Horizon as Critical Phenomenon, JHEP 09 (2016) 044 [arXiv: 1603. 08509] [INSPIRE].

[50] S.-S. Lee, Emergent gravity from relatively local Hamiltonians and a possible resolution of the black hole information puzzle, JHEP 10 (2018) 043 [arXiv:1803.00556] [INSPIRE].

[51] C. Fronsdal, Massless Fields with Integer Spin, Phys. Rev. D 18 (1978) 3624 [InSPIRE].

[52] E.S. Fradkin and M.A. Vasiliev, Cubic Interaction in Extended Theories of Massless Higher Spin Fields, Nucl. Phys. B 291 (1987) 141 [inSPIRE].

[53] I.R. Klebanov and A.M. Polyakov, AdS dual of the critical $O(N)$ vector model, Phys. Lett. B 550 (2002) 213 [hep-th/0210114] [INSPIRE].

[54] X. Bekaert, N. Boulanger and P. Sundell, How higher-spin gravity surpasses the spin two barrier: no-go theorems versus yes-go examples, Rev. Mod. Phys. 84 (2012) 987 [arXiv: 1007.0435] [INSPIRE].

[55] E. Mintun and J. Polchinski, Higher Spin Holography, RG, and the Light Cone, arXiv:1411.3151 [INSPIRE].

[56] R.G. Leigh, O. Parrikar and A.B. Weiss, Holographic geometry of the renormalization group and higher spin symmetries, Phys. Rev. D 89 (2014) 106012 [arXiv:1402.1430] [InSPIRE].

[57] R.G. Leigh, O. Parrikar and A.B. Weiss, Exact renormalization group and higher-spin holography, Phys. Rev. D 91 (2015) 026002 [arXiv:1407.4574] [INSPIRE].

[58] A. Lewkowycz and J. Maldacena, Generalized gravitational entropy, JHEP 08 (2013) 090 [arXiv: 1304.4926] [INSPIRE].

[59] A.N. Petrov, Classical and Quantum Gravity Research, M.N. Christiansen and T.K. Rasmussen eds., Nova Science Publishers, New York U.S.A. (2008).

[60] K.-S. Kim, Emergent geometry in recursive renormalization group transformations, Nucl. Phys. B 959 (2020) 115144 [arXiv: 2004.09997] [INSPIRE].

[61] K.-S. Kim, Geometric encoding of renormalization group $\beta$-functions in an emergent holographic dual description, Phys. Rev. D 102 (2020) 026022 [arXiv:2003.00281] [INSPIRE].

[62] K.-S. Kim, S.B. Chung, C. Park and J.-H. Han, Emergent holographic description for the Kondo effect: Comparison with Bethe ansatz, Phys. Rev. D 99 (2019) 105012 [arXiv: 1705. 06571] [INSPIRE].

[63] R.S. Hamilton, Hamilton: Three-manifolds with positive Ricci curvature, J. Diff. Geom. 17 (1982) 255.

[64] D.H. Friedan, Nonlinear Models in Two + Epsilon Dimensions, Annals Phys. 163 (1985) 318 [INSPIRE].

[65] B. Chow and D. Knopf, Mathematical Surveys and Monographs. Vol. 110: The Ricci Flow: An Introduction, AMS Press, Providence U.S.A. (2004).

[66] G. Perelman, The Entropy formula for the Ricci flow and its geometric applications, math/0211159 [INSPIRE].

[67] E. Woolgar, Some Applications of Ricci Flow in Physics, Can. J. Phys. 86 (2008) 645 [arXiv: 0708.2144] [INSPIRE]. 
[68] M. Headrick and T. Wiseman, Ricci flow and black holes, Class. Quant. Grav. 23 (2006) 6683 [hep-th/0606086] [INSPIRE].

[69] E. Kiritsis, W. Li and F. Nitti, Holographic RG flow and the Quantum Effective Action, Fortsch. Phys. 62 (2014) 389 [arXiv:1401.0888] [InSPIRE].

[70] S. Jackson, R. Pourhasan and H. Verlinde, Geometric RG Flow, arXiv:1312.6914 [INSPIRE].

[71] M. Plischke and B. Bergersen, Equilibrium Statistical Physics, World Scientific Publishing, London U.K. (2006).

[72] R.L. Arnowitt, S. Deser and C.W. Misner, Dynamical Structure and Definition of Energy in General Relativity, Phys. Rev. 116 (1959) 1322 [InSPIRE].

[73] S. Corley, A Note on holographic Ward identities, Phys. Lett. B 484 (2000) 141 [hep-th/0004030] [INSPIRE].

[74] K.-S. Kim, Emergent dual holographic description for interacting Dirac fermions in the large $N$ limit, Phys. Rev. D 102 (2020) 086014 [arXiv: 2003.00291] [inSPIRE].

[75] T.W. Appelquist, M.J. Bowick, D. Karabali and L.C.R. Wijewardhana, Spontaneous Chiral Symmetry Breaking in Three-Dimensional QED, Phys. Rev. D 33 (1986) 3704 [INSPIRE].

[76] G. Policastro, D.T. Son and A.O. Starinets, From AdS/CFT correspondence to hydrodynamics, JHEP 09 (2002) 043 [hep-th/0205052] [INSPIRE].

[77] G. Policastro, D.T. Son and A.O. Starinets, From AdS/CFT correspondence to hydrodynamics. 2. Sound waves, JHEP 12 (2002) 054 [hep-th/0210220] [INSPIRE].

[78] P. Kovtun, D.T. Son and A.O. Starinets, Holography and hydrodynamics: Diffusion on stretched horizons, JHEP 10 (2003) 064 [hep-th/0309213] [INSPIRE].

[79] P. Kovtun, D.T. Son and A.O. Starinets, Viscosity in strongly interacting quantum field theories from black hole physics, Phys. Rev. Lett. 94 (2005) 111601 [hep-th/0405231] [INSPIRE]. 